\title{
Localised Waves \\ Tightest Focus, Lorentz Transformation, and Polarization Singularities of Non-Paraxial \\ Beams
}

by

Petar Andrejić

A thesis

submitted to the Victoria University of Wellington

in fulfilment of the

requirements for the degree of

Master of Science

in Physics.

Victoria University of Wellington

2018 



\begin{abstract}
I explore the limits of how tightly a beam can be focused, and derive a focal parameter for scalar beams that can be symbolically evaluated for most beams, and is guaranteed to be convergent for physical beams, that compares peak intensity to the total intensity in the beam profile. I argue that this parameter is superior to spot size, and use this to derive a rigorous limit of focusing for scalar beams. A particular beam known as the proto-beam achieves this tightest focus possible. I show the generalisation of this measure to electromagnetic beams, and place a lower bound on the focal extent of electromagnetic beams. I also propose the use of exponential regulators as alternatives to moment based measures, as a solution to the convergence issues created by the power law decay of exact solutions.

I explore the Doppler shift for finite beams, and how monochromatic beams become polychromatic under a Lorentz boost. The local frequency is also explored, and I show that a deviation of the local frequency from the Doppler frequency will occur due to wavelength broadening near the focus.

Lekner and I examine a beam that closely approximates a paraxial Gaussian beam radially, and examine the phase singularities for optical beams that occur near the zeros of the beams wavefunction. We also investigate attempts to find exact solutions with Gaussian profiles, and show that this is impossible; any such beam will be evanescent and exponentially grow. Finally, I investigate the property of finite classical electromagnetic pulses having a zero momentum frame, and show that for quantum single photon pulses this property holds for the expectation value. I show that any individual measurement however, still measures a light-like four-momentum for the photon.
\end{abstract}




\section{Acknowledgements}

I would like to extend my sincere gratitude to my supervisor, Professor John Lekner, for allowing me the opportunity and independence to explore the topics that have most interested me. His feedback and contributions to this thesis have been immensely valuable

I would also like to extend my gratitude to my parents, Nebojša and Jasmina, as well as my sister Natalija, and my brother-in-law Alexander, who have supported me greatly throughout my five years of study at Victoria. Natalija in particular deserves special thanks for proof-reading my spelling and grammar.

Finally, I am grateful to the financial assistance provided by Victoria University of Wellington's scholarships, which have funded the entirety of my studies. 


\section{Contents}

1 Introduction 1

1.1 Motivation . . . . . . . . . . . . . . . . . . . . . 1

1.2 Wave equations and beams $\ldots \ldots \ldots \ldots \ldots \ldots$

1.3 Paraxial optics . . . . . . . . . . . . . . . . . . . 6

1.4 Zeros and phase singularities . . . . . . . . . . . . . 8

1.5 Electromagnetic beams . . . . . . . . . . . . . . . . . . 14

1.6 Operator algebra $\ldots \ldots \ldots \ldots \ldots$

1.7 Academic contributions . . . . . . . . . . . . . . . 21

2 Focusing limits of finite beams 23

2.1 Introduction . . . . . . . . . . . . . . . . . . . . 23

2.2 Beam invariants . . . . . . . . . . . . . . . . . . . . . . 24

2.3 Focusing of beams $\ldots \ldots \ldots \ldots \ldots \ldots \ldots$

2.4 Axial focal extent . . . . . . . . . . . . . . . . . . . 32

2.5 Radial focusing . . . . . . . . . . . . . . . . . . 36

2.6 Optical focusing . . . . . . . . . . . . . . . . . . . 38

2.7 Transverse modes . . . . . . . . . . . . . . . . . . . . . . . . . . 40

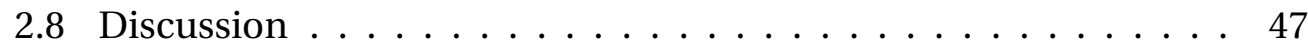

3 Nonexistence of Gaussian profiles

3.1 Introduction . . . . . . . . . . . . . . . . . . . . . . . . 54

3.2 An exact solution and its properties . . . . . . . . . . . . 57

3.3 The ratio of $\psi_{G}(\rho, z)$ to $\psi_{b}(\rho, z) \ldots \ldots \ldots \ldots \ldots$ 
3.4 Proof that an exact match to $\psi_{G}(0, z)$ does not exist . . . . . . . . 62

3.5 An exact solution with Gaussian profile in the focal plane does not exist . . . . . . . . . . . . . . . . . . . . . . 64

3.6 Discussion $\ldots \ldots \ldots \ldots \ldots$. . . . . . . . . . . . . 65

$3.7 \quad$ Divergence angle of the $\psi_{b}(\rho, z)$ beam $\ldots \ldots \ldots 6$

3.8 Nonexistence of Bessel-Gauss . . . . . . . . . . . . . . . . 67

3.9 Paraxial to exact . . . . . . . . . . . . . . . . 68

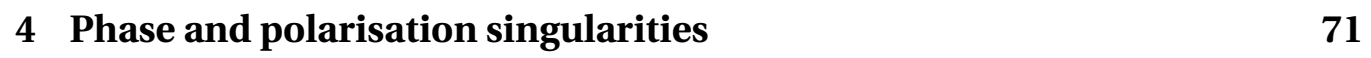

4.1 Introduction . . . . . . . . . . . . . . . . . . . . . . . . 72

$4.2 \quad$ Properties of the beam wavefunction $\psi_{b}(\rho, z) \ldots \ldots$. . . . . 75

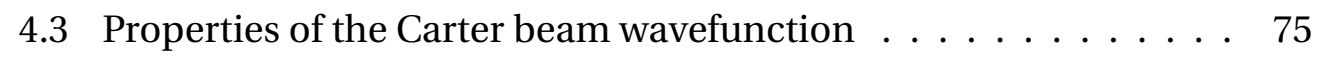

4.4 Polarization of 'LP' beams $\ldots \ldots \ldots$. . . . . . . . . . . 78

4.5 Polarization properties of 'LP' beams based on $\psi_{0}, \psi_{b}$ and $\psi_{C}$. . 80

4.6 Discussion . . . . . . . . . . . . . . . . . . . . . . . . 81

\begin{tabular}{|lll}
\hline 5 & Reflection of focused beams & 91
\end{tabular}

5.1 Introduction . . . . . . . . . . . . . . . . . . 91

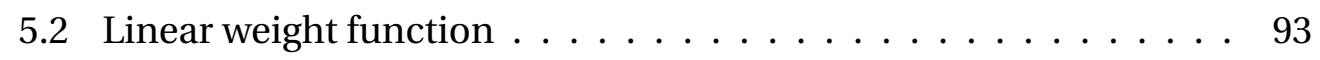

5.3 Bessel beam incident at an angle . . . . . . . . . . . . . . 95

5.4 General beam-forms . . . . . . . . . . . . . . . . . . . 101

5.5 Proto-beam oblique transmittance . . . . . . . . . . . . . 102

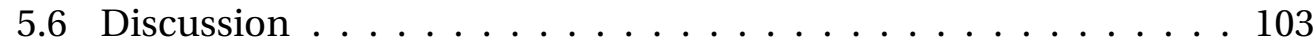

6 Lorentz transformation of beams $\quad 113$

6.1 Introduction . . . . . . . . . . . . . . . . . . . 113

6.2 Generalised Bessel Beam . . . . . . . . . . . . . . . 115

6.3 Lorentz transformation of wave envelopes . . . . . . . . . . . 117

6.4 Paraxial beams . . . . . . . . . . . . . . . . . . . 119

6.5 Scattering considerations . . . . . . . . . . . . . 123

6.6 Discussion . . . . . . . . . . . . . . . . . 127 
\begin{tabular}{|lll}
7 & Covariant Hertz Potentials & 129
\end{tabular}

7.1 Introduction . . . . . . . . . . . . . . . . . . . . . . 130

7.2 'CP' pulse . . . . . . . . . . . . . . . . . . . . . . . . 134

$7.2 .1 \quad$ Zero Momentum Frame . . . . . . . . . . . . . . . . . . . 134

7.2 .2 Angular momentum . . . . . . . . . . . . 136

7.3 TE+iTM pulse . . . . . . . . . . . . . . . . . . 137

7.3 .1 Zero momentum frame . . . . . . . . . . . . . . 138

7.4 Discussion . . . . . . . . . . . . . . . . . . . . . 139

7.5 Appendix: 'CP' momentum densities . . . . . . . . . . . . . 140

8 Single photon states $\quad 141$

8.1 Introduction . . . . . . . . . . . . . . . . . . . . . . . . 141

8.2 Construction of quantum state $\ldots \ldots \ldots$. . . . . . . . . . . 142

9 Conclusions 147

9.1 Focusing of finite beams . . . . . . . . . . . . . . . . . . . 147

9.2 Zeros of wavefunctions . . . . . . . . . . . . . . . . . . . . . . 149

9.3 Reflection of beams . . . . . . . . . . . . . . . . . . 151

9.4 Lorentz transformation of localised waves $\ldots \ldots \ldots$. . . . . . . 152

9.5 Energy and momentum of single photon pulses . . . . . . . . 153 


\section{Chapter 1}

\section{Introduction}

\subsection{Motivation}

Many physical systems are described by the wave equation, and other related equations. For example, particle beams are described by the Schrödinger, Dirac or Klein Gordon equations, Maxwell's equations for electromagnetism in free space include the wave equation, and sound and linearised hydrodynamics is also governed by the wave equation. In many cases, it is adequate to use plane wave analysis. As we shall see, locally, solutions of wave equations can be described by plane waves, so if an experiment involves the interaction of waves with a particle much smaller than the wavelength of the wave, the local properties, and hence plane wave analysis, will suffice. However, there are applications where the feature size of the object interacting with the wave are comparable in size to the wavelength. For example, electron beams in electron microscopes, or the optics of photo-lithography, where commercially available feature sizes are as of the time of writing as low as $10 \mathrm{~nm}$. In such regimes, plane wave descriptions will not be adequate, nor will the commonly used paraxial approximation for beams. As such, study of exact solutions of the aforementioned wave equations are required to understand the properties of such tightly focused beams. 


\subsection{Wave equations and beams}

The focus of this thesis is on the properties of localised waves, primarily dealing with tightly focused beams, however briefly touching on localised pulses as well. Define the wave-function, $\psi$ to be the relevant physical scalar field under consideration. For quantum mechanical waves this is the probability density, for acoustic beams the pressure field, and so forth. In addition to the solutions of wave equation itself,

$$
\nabla^{2} \psi-\frac{1}{c^{2}} \frac{\partial^{2} \psi}{\partial t^{2}}=0
$$

we will also examine quantum mechanical scalar waves, which in free space satisfy Schrödinger's equation [1],

$$
-\frac{\hbar^{2}}{2 m} \psi=i \hbar \frac{\partial \psi}{\partial t}
$$

or the Klein Gordon equation |2, 3, 4|,

$$
\nabla^{2} \psi-\frac{1}{c^{2}} \frac{\partial^{2} \psi}{\partial t^{2}}+\frac{m^{2} c^{2}}{\hbar^{2}} \psi=0
$$

The unifying feature of all three above equations is their form for monochromatic beams: assuming $\psi(\boldsymbol{r}, t)=\psi(\boldsymbol{r}) e^{-i \omega t}$, we have

$$
\nabla^{2} \psi+k^{2} \psi=0
$$

with $k$ equal to $\omega / c, \sqrt{2 m \omega / \hbar}$ and $\sqrt{\hbar^{2} \omega^{2}+m^{2} c^{4}} / \hbar c$ for the wave equation, Schrödinger and Klein-Gordon equations respectively. As a result, for monochromatic beams the same mathematical solutions will apply universally to the above situations, albeit with different physical interpretations, and different phase velocities.

We will begin with a brief exposition of how Fourier analysis and operator algebra applies to the analysis of beams. While this is most likely already familiar to the reader, we wish to emphasise the application to beams specifically. More detail can be found in any of the various textbooks on Fourier analysis, 
Quantum mechanics, or Hilbert space theory. Authors we suggest include Griffiths [1], Srednicki [4], Greiner [3, 2] and Young [5]. As a linear partial differential equation, for a given coordinate system the Helmholtz equation will have a family of fundamental solutions (eigenmodes), with different symmetries. For Cartesian coordinates, we will of course have plane waves, $e^{i \boldsymbol{k} \cdot \boldsymbol{r}}$, with any solution being expressible as a superposition of plane waves,

$$
\psi(\boldsymbol{r})=\int_{\mathscr{S}} \mathrm{dA} f(\hat{k}) e^{i \boldsymbol{k} \cdot \boldsymbol{r}}
$$

where $\mathscr{S}$ is a sphere of radius $k$ in Fourier space, and $f$ a distribution over it. This can of course be expressed using spherical coordinates as

$$
\psi(\boldsymbol{r})=\int_{0}^{\pi} \int_{-\pi}^{\pi} \sin \alpha \mathrm{d} \alpha \mathrm{d} \beta f(\alpha, \beta) e^{i \boldsymbol{k}(\alpha, \beta) \cdot \boldsymbol{r}},
$$

with $\boldsymbol{k}$ being the wavevector in spherical coordinates $\alpha, \beta$. Defining the wavevector operator to be $\hat{k}=-i \nabla$, we can see that plane waves are eigenfunctions of this operator.

Cylindrical coordinates $\rho, \phi, z$ give rise to Bessel beam solutions,

$$
e^{i m \phi} e^{i k z \cos \alpha} J_{m}(k \rho \sin \alpha)
$$

Defining the angular wavevector operator as $\hat{l}=\hat{r} \times \hat{k}$, in cylindrical and spherical coordinates we will have

$$
\hat{l}_{z}=-i \frac{\partial}{\partial \phi}
$$

Bessel beams are the simultaneous eigenfunctions of $\hat{l}_{z}$ and $\hat{k}_{z}$. For quantum particle beams $\hat{l}$ and $\hat{k}$ are proportional to the angular momentum and linear momentum operators respectively [1], and Bessel beams represent the eigenmodes with definite $z$ momentum and angular momentum. Cylindrical coordinates also give rise to Bessel function solutions with a $Y_{m}(\kappa \rho)$ radial dependence; these are not physical for free space propagation as they are singular along the beam axis.

Geometrically, the Fourier space of the Helmholtz equation, the domain of the Fourier transform of the equation, is the surface of a sphere of radius $k$. 
For particle beams, the modulus $|\psi(\boldsymbol{r})|^{2}$ gives the probability density, with the usual normalisation being $\int_{\mathbb{R}^{3}} \mathrm{~d}^{3} r|\psi|^{2}=1$. However, solutions of the Helmholtz equation always have a divergent volume integral: the domain of the Fourier transform on $\mathbb{R}^{3}$ is $\mathbb{R}^{3}$, and the integral of $|\psi|^{2}$ over $\mathbb{R}^{3}$ is the integral of the Fourier transform, $|f|^{2}$ over $\mathbb{R}^{3}$ (Plancharel's theorem).

For a solution $\psi$ of the Helmholtz equation, the Fourier transform,

$$
\int_{\mathbb{R}^{3}} \mathrm{~d}^{3} r \psi(\boldsymbol{r}) e^{i \boldsymbol{k} \cdot \boldsymbol{r}}
$$

is a layer on a sphere, therefore in spherical coordinates we have the Fourier transform given by $f(\alpha, \beta) \delta\left(k-k_{0}\right)$, for some arbitrary distribution $f(\alpha, \beta)$. As a result, the integral over all of Fourier space will involve the integral of the square of $\delta\left(k-k_{0}\right)$, which is divergent. Physically, an ideal beam extends to infinity in at least one direction.

Bessel beams can be expressed as superpositions of plane waves via the cylindrical plane wave expansion. First we write both the wavevector and position vector of a plane wave in spherical coordinates:

$$
e^{i \boldsymbol{k} \cdot \boldsymbol{r}}=\exp (i k r(\cos \theta \cos \alpha+\sin \theta \sin \alpha(\cos \phi \cos \beta+\sin \phi \sin \beta))),
$$

where $\alpha, \beta$ are the wavevector coordinates, $\theta, \phi$ the position coordinates. Next, we note that $\cos \beta \cos \phi+\sin \beta \sin \phi=\cos (\beta-\phi)$. With $z=r \cos \theta, \rho=r \sin \theta$ we have

$$
e^{i \boldsymbol{k} \cdot \boldsymbol{r}}=e^{i k z \cos \alpha} e^{i k \rho \sin \alpha \cos (\beta-\phi)} .
$$

The generating function for Bessels is [6]

$$
e^{\frac{1}{2} \xi\left(\tau-\tau^{-1}\right)}=\sum_{m=-\infty}^{\infty} \tau^{m} J_{m}(\xi) .
$$

In our case we can set $\xi=k \rho \sin \alpha, \tau=i e^{i(\beta-\phi)}$. This will give

$$
e^{i \boldsymbol{k} \cdot \boldsymbol{r}}=e^{i k z \cos \alpha} \sum_{m=-\infty}^{\infty} i^{m} e^{i m(\beta-\phi)} J_{m}(k \rho \sin \alpha) .
$$

We can obtain a single mode from this by noting

$$
\int_{0}^{2 \pi} e^{i\left(m-m^{\prime}\right) \beta} \mathrm{d} \beta=2 \pi \delta_{m, m^{\prime}} .
$$




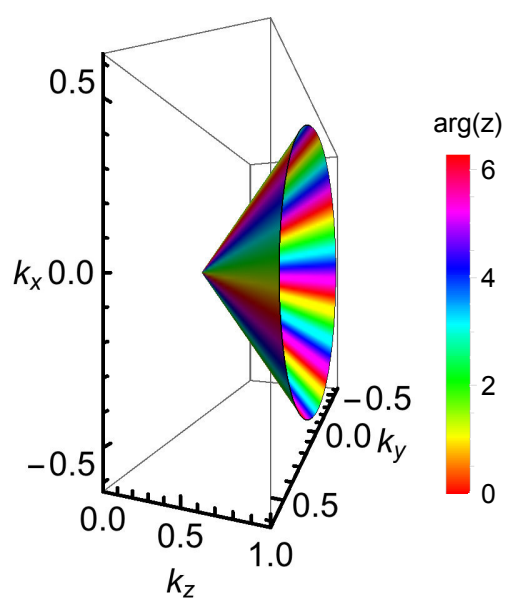

Figure 1.1: Bessel beams can be viewed as superpositions of plane waves with wavevectors all lying on a cone. Here, we have the surface in Fourier space swept out by the Bessel $m=7$ beam's wavevectors, with a conical angle of 0.5 radians. The shading gives the phase of the associated wavevector.

Thus, we have

$$
e^{i m \phi} e^{i k z \cos \alpha} J_{m}(k \rho \sin \alpha)=\frac{1}{2 \pi} \int_{0}^{2 \pi} e^{i \boldsymbol{k} \cdot \boldsymbol{r}} e^{-i m \beta} \mathrm{d} \beta
$$

The geometrical interpretation is simple: Bessel beams are formed by superpositions of plane waves with wave-vectors lying on a cone of angle $\alpha$, each multiplied by a phase of $e^{-i m \beta}$, Figure 1.1.

For arbitrary wavefunctions, we can define a local wavevector, and local wavelength [7, sec. 8.1]. In modulus-phase form we have

$$
\psi(\boldsymbol{r})=M(\boldsymbol{r}) e^{i S(\boldsymbol{r})}
$$

A series expansion of the phase gives in the vicinity of a point $p$

$$
S(\boldsymbol{r})=S(\boldsymbol{p})+\left.(\boldsymbol{r}-\boldsymbol{p}) \cdot \nabla S\right|_{p}+\mathscr{O}\left((r-p)^{2}\right) .
$$

Hence, sufficiently close to $p$, the variation of the phase is given by that of a plane wave with wavevector $\nabla S$. This implies that locally, the wavevector is given by the gradient of the phase. 
This can be generalised for polychromatic wave-packects. Regardless of the differential equation satisfied by the wavepacket, for example any of equations (1.2.2), (1.2.1) or (1.2.3), the wave-function can be written as a modulus times phase factor,

$$
\psi=M\left(x^{\mu}\right) e^{i S\left(x^{\mu}\right)}
$$

The expansion for the phase then gives

$$
S\left(x^{\mu}\right)=S\left(p^{\mu}\right)+\left.\left(x^{\mu}-p^{\mu}\right) \partial_{\mu} S\right|_{p}+\mathscr{O}\left((x-p)^{2}\right) \text {. }
$$

As well as the gradient of the phase giving the local wavevector, the time derivative gives the local frequency [7, sec. 8.1].

In Chapter 6 we discuss the Lorentz transformation of beams and pulses, in particular the associated Doppler shift. In general, monochromatic beams will be transformed into polychromatic beams. This is because monochromatic beams can be considered to be superpositions of plane waves in different directions; the Doppler shift in frequency for each directional component will therefore differ. This can complicate scattering problems for scatterers that are moving relative to a beam. For scatterers much larger than the focal extent, we would usually expect the global properties to be more important, thus the wavevector and frequency distributions will play a significant role. In contrast, for point-like scatterers the particle size is much smaller than the focal extent of the beam, and we can approximate the beam as a plane wave in the vicinity of the particle. As a result, the local frequency and wavevector of the beam will play a significant role. We discuss this further in Chapter 6 .

\subsection{Paraxial optics}

While the focus of this thesis is exact solutions for highly localised beams, it is important to understand paraxial beams, their areas of validity, and their properties, in order to distinguish the unique features of exact beams. The paraxial approximation is closely related to geometric optics. The paraxial equation can be obtained via the 'slowly varying envelope' approximation. We have seen that 
monochromatic waves satisfy the Helmholtz equation. For the rest of this thesis we will default to writing the beam's propagation direction (the beam axis) as $z$. We can always write a wavefunction as $\psi(\boldsymbol{r})=u(\boldsymbol{r}) e^{i k z}$, for some 'envelope' $u$. Plugging this into the Helmholtz equation gives the following equation for $\mathrm{u}$ :

$$
\nabla^{2} u+2 i k \frac{\partial u}{\partial z}=0
$$

The assumption is that the variation of the envelope is over a far greater length scale than the wavelength, $2 \pi / k$, and hence $\frac{\partial^{2} u}{\partial z^{2}} \ll 2 i k \frac{\partial u}{\partial z}$. This second $z$ derivative is therefore dropped, to give the paraxial Helmholtz equation

$$
\nabla_{\perp}^{2} u+2 i k \frac{\partial u}{\partial z}=0 .
$$

Here, $\nabla_{\perp}$ is the perpendicular gradient operator, such that

$$
\nabla_{\perp}=\frac{\partial}{\partial x} \hat{x}+\frac{\partial}{\partial y} \hat{y}, \quad \nabla_{\perp}^{2}=\frac{\partial^{2}}{\partial x^{2}}+\frac{\partial^{2}}{\partial y^{2}} .
$$

Both the paraxial and exact Helmholtz equations permit Bessel beam solutions,

$$
\psi(\rho, z) e^{i q z} J_{0}(\kappa \rho) .
$$

However, the relation of $q$ to $\kappa$ is different for the two equations. We have $q(k, \kappa)=\sqrt{k^{2}-\kappa^{2}}$ and $q_{p}(k, \kappa)=k-\frac{\kappa^{2}}{2 k}$ for the exact and paraxial solutions respectively. We can see that if $\kappa$ is small, a Taylor series at the origin gives

$$
q(k, \kappa)=k-\frac{\kappa^{2}}{2 k}+\mathscr{O}\left(\kappa^{4}\right)=q_{p}(k, \kappa)+\mathscr{O}\left(\kappa^{4}\right) .
$$

This is a physically equivalent assumption: the smaller $\kappa$ is, the longer the range of variation in $J_{0}(\kappa \rho)$, which gives a 'slowly varying envelope'. Geometrically, since $\kappa=k \sin \alpha$, we can see that the paraxial approximation is equivalent to the Fourier transform of the beam being restricted to a small conical angle $\alpha$.

The most commonly used paraxial solution is the Gaussian beam [8, 9],

$$
\psi_{G}(\rho, z)=\frac{b}{b+i z} \exp \left(i k z-\frac{k \rho^{2}}{2(b+i z)}\right) .
$$


This represents a beam propagating along $z$, converging to a focus at the origin. The parameter $b$, with dimensions of length, determines the focal extent of the beam, and is sometimes referred to as the Rayleigh length, $z_{R}[\overline{9} \mid$. Other commonly defined features include the 'beam radius', the radius at which the modulus drops to $e^{-2}$ that of the peak,

$$
w(z)=\sqrt{\frac{2}{k b}\left(b^{2}+z^{2}\right)} .
$$

with the focal plane value $w_{0}=\sqrt{2 b / k}$ being referred to as the 'beam waist'. $b=z_{R}$ is the length along the beam axis at which the beam radius is $\sqrt{2} w_{0}$, and the axial modulus is half of the peak. The phase of $b+i z$ is referred to as the 'Gouy phase', $\Phi(z)=\arctan (z / b)$, and results in an increase in the local wavevector close to the focus. Finally, the radius of curvature of the wavefronts is given by $R(z)=z\left(1+(b / z)^{-2}\right)$. Many authors have been interested in the non-paraxial generalisation of Gaussian beams. In particular, Takenaka [10], Seshadri [11], Wünsche [12] and April [13] have all attempted to find exact solutions of the Helmholtz equation that match Gaussian, Laguerre Gaussian, and even Bessel-Gauss (Gaussian times $J_{0}(k \rho)$ ) beams in the focal plane. In Chapter 3 we give a paper, produced in the course of this thesis, co-authored with John Lekner, in which we investigate the space of exact solutions to the Helmholtz equation, and show that these attempts are flawed. While it is possible to match an exact solution to the aforementioned beams in the focal plane, this will result in an evanescent beam, one that grows exponentially along the propagation direction. As such, these solutions cannot represent a physical beam.

\subsection{Zeros and phase singularities}

We shall be making use of the domain colouring method of visualisation for complex valued functions. As this is a less common visualisation technique, we shall take time to explain how to interpret these plots. In the HSV model of colour, the hue $(\mathrm{H})$ parameter maps the hue of a colour cyclically to a circle. 


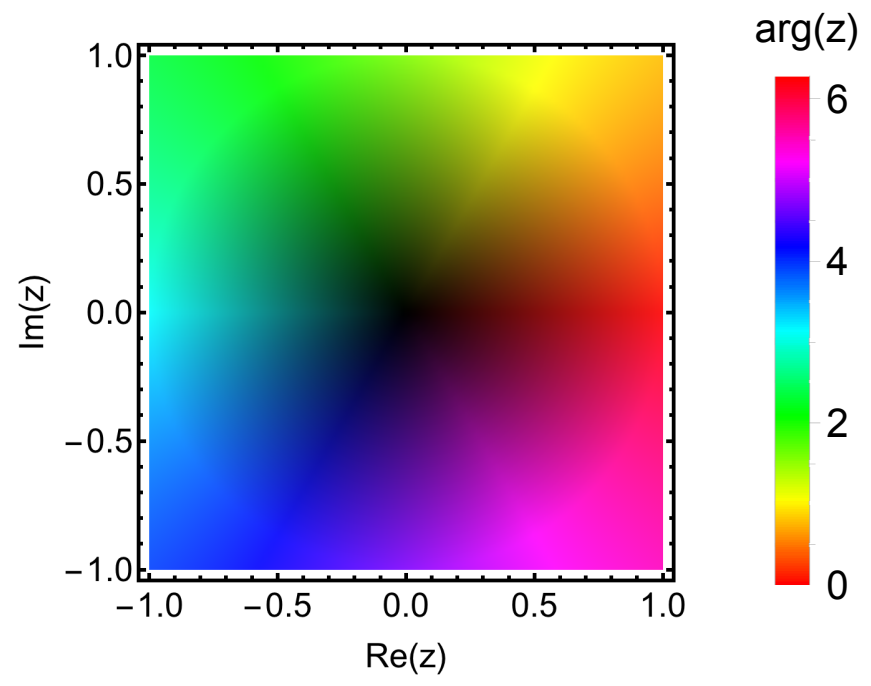

Figure 1.2: Domain colouring of identity function $z \rightarrow z$ in the complex plane. Note how the colour gives the phase of the complex number, and the shading, the magnitude.

The saturation (S) parameter controls how far the colour is from white (0 being pure white, and 1 fully saturated), and the value (V) parameter controls the luminance. As the phase of a complex number is mapped to a circle, we can thus map the phase to hue. For readability it is useful to leave the saturation at maximum, thus the modulus is mapped instead to the value. The value parameter is 0 for black, and 1 for maximum luminance. To help with the interpretation, Figure 1.2 gives the domain colouring of the identity function in $\mathbb{C}$.

If a function spans many orders of magnitude in a region, then an appropriate modulus scaling should be introduced. This scaling function will in general need to be matched to the growth or decay rate of the function. As beams have exponential decay, or greater, it will be useful to scale the modulus logarithmically. We shall use a 'terraced' scaling method, with the scaling function being

$$
s(z)=(2 \ln |z| \bmod 1)^{0.2} .
$$

This scaling first scales the modulus squared (intensity) logarithmically, with the modulo 1 making the luminosity periodic (or 'terraced'): the luminosity 
decreases as the intensity decreases, and reaches its minimum every time the intensity scales by $e^{-1}$.

At this contour there is a jump in luminosity from minimum to maximum, and the process repeats. This helps illustrate the shape of the beam by providing hard contour edges. The 0.2 power scaling is to ensure the luminance is not too dark. If the beam is normalised to unity the second luminance contour corresponds to the radius at which the beam intensity has decreased to $e^{-2}$ of the maximum, which is a commonly used measure of the focal extent and spot size.

Figure 1.3 illustrates domain colour plots for complex polynomials, demonstrating a key feature of zeros of complex functions: the phase singularity. The phase of a complex number is a continuous mapping from $\mathbb{C} \backslash\{0\} \rightarrow \mathbb{S}^{1}$, which can of course be parametrized by the angle $\theta \in[0,2 \pi)$. As an example, figure 1.4 gives the domain colouring of the Gaussian beam.

Note the mapping is undefined when the complex number $z$ is zero. Around the zero, the phase circulates, making $m$ complete cycles from 0 to $2 \pi$. This gives an associated charge to the zero: the number of times the phase cycles, and the direction, which can be represented by an integer $m$, positive for anticlockwise.

For a beam, phase singularities are most often discussed in association with higher order angular momentum modes. To illustrate, consider the paraxial Laguerre-Gauss modes. These are solutions to the paraxial equation that are eigenfunctions of $\frac{\partial}{\partial \phi}$. The simplest of these are given by

$$
\psi_{0, m}=\left(\frac{\rho}{b+i z}\right)^{|m|} e^{i m \phi} \psi_{G},
$$

with the full set given by

$$
\psi_{n, m}=\psi_{0, m} e^{-2 i n \arctan (z / b)} L_{n}^{|m|}\left(\frac{k b \rho^{2}}{b^{2}+z^{2}}\right) .
$$

Here, $L_{n}^{|m|}$ is a generalised Laguerre polynomial. For $|m|>0$, these beams have the property of being zero along the beam axis, with the charge of the singularity given by $m$. For particle beams, they have an angular momentum of 


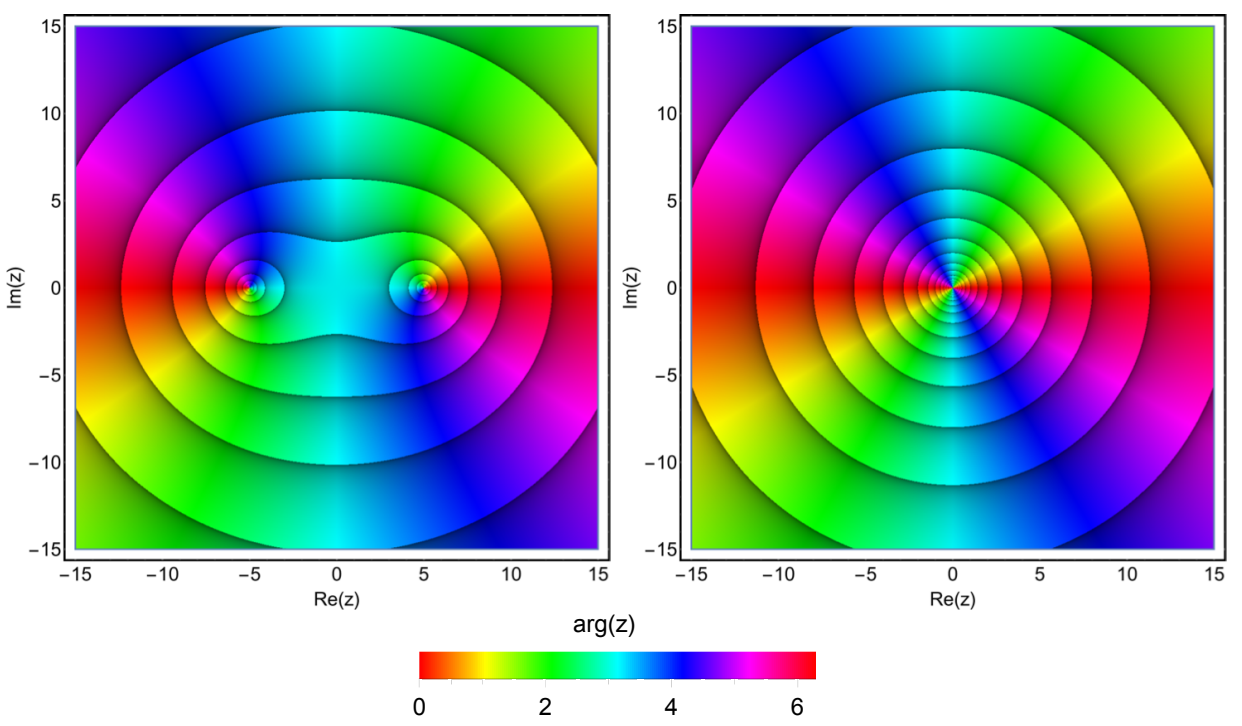

Figure 1.3: Domain colour plot for $(z-1)(z+1)$, left, and $z^{2}$, right. Note the phase singularity at the zeros: the phase of a complex number $\xi$ is undefined at $\xi=0$, and in the neighbourhood of the zero the phase circulates from 0 to $2 \pi$. The zero has with it an associated charge: the number of times the phase circulates on a closed loop around the zero. For $(z-1)(z+1)$ the charge is \pm 1 at each zero, for $z^{2}$ it is 2 . Note for these plots the modulus was scaled via $s(z)=$ $\left(\log _{2}|z| \bmod 1\right)^{0.2}$

$L_{z}=\hbar m$. Figures 1.6 and 1.5 give the domain colour of the $p=0, m=2$ beam in both the focal plane $z=0$ and the half-plane $x>0, y=0$. Clearly visible is the phase singularity along the beam axis, with the phase making two full cycles around it. Higher order modes with $p>0$ have a different nature of zero, being zero along a series of infinite cylindrical sheets. These zeros instead function as a 'shear' phase singularity, with a $\pi$ jump in phase across it, but no circulation associated. This is illustrated in figures 1.5 and 1.6. Bessel beams have an infinity of sheets zeros, however Bessel beams are not radially square integrable and so are not useful for modelling beam propagation outside of waveguides.

In addition to axial lines, or cylindrical sheets, certain solutions admit 'ring' zeros, where the wave-function is zero on a closed loop. These zeros have an 


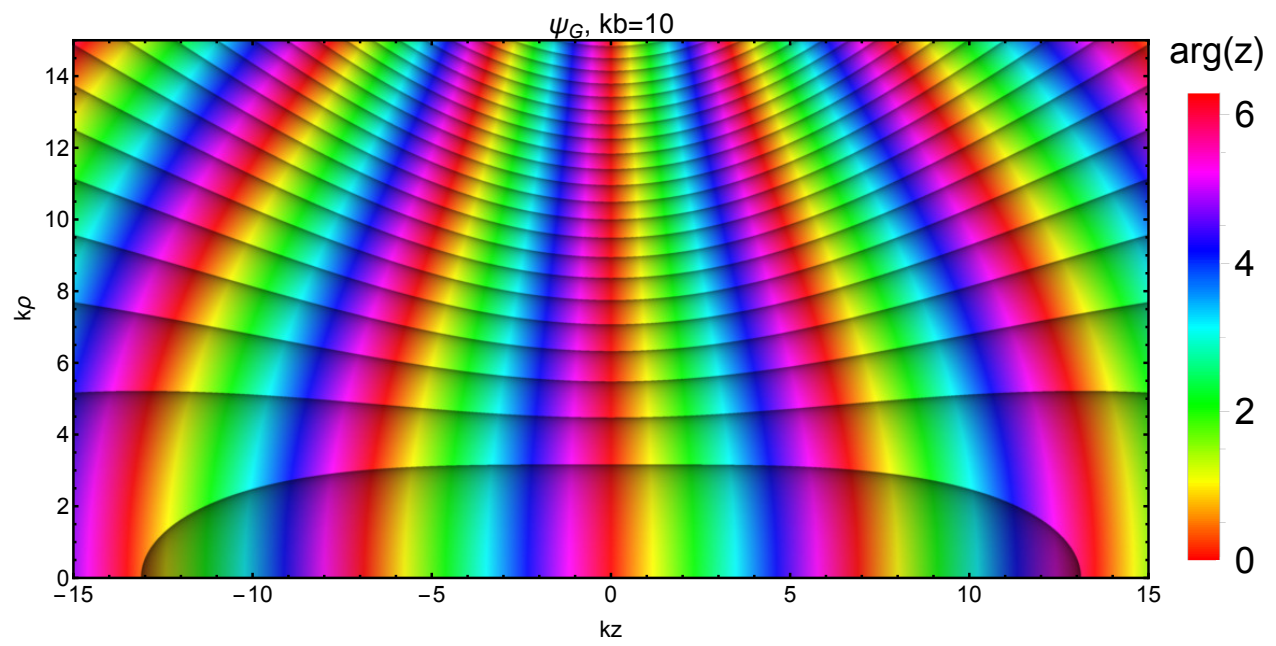

Figure 1.4: Domain colouring of the Gaussian beam in $\rho z$ plane, for $k b=10$. The beam width is defined to be the radius at which the intensity drops to $e^{-2}$ of the value at the origin, which can be seen here as the second shaded intensity contour, at around $k \rho=5$. There are no zeros, and the wavefronts meet at infinity.

associated vorticity around them, reminiscent perhaps of the magnetic field lines of around a loop of current. Consider a disk perpendicular to the zero ring at a given point: the loop intersects this disk to make an isolated zero. As such, the phase circulates, with an associated charge.

For particle beams, as the probability current is proportional to the gradient of the phase, these ring zeros are associated with a toroidal vorticity in the probability current (Figure 1.7). However, unlike axial zeros, there is no net angular momentum associated with these zeros. As a simple example of a beam with ring zeros, consider the 'proto-beam' as examined in Lekner, 2016 [14]:

$$
\psi_{0}(\rho, z)=\frac{2}{k^{2}} \int_{0}^{k} \mathrm{~d} q q e^{i q z} J_{0}(\kappa \rho) .
$$

The focal plane value has a closed form expression, and is given by

$$
\psi_{0}(\rho, 0)=2 J_{1}(k \rho) / k \rho \text {. }
$$

This is zero when $J_{1}(k \rho)=0$, at $k \rho=3.83171,7.01559,10.1735 \ldots$ Figure 1.8 

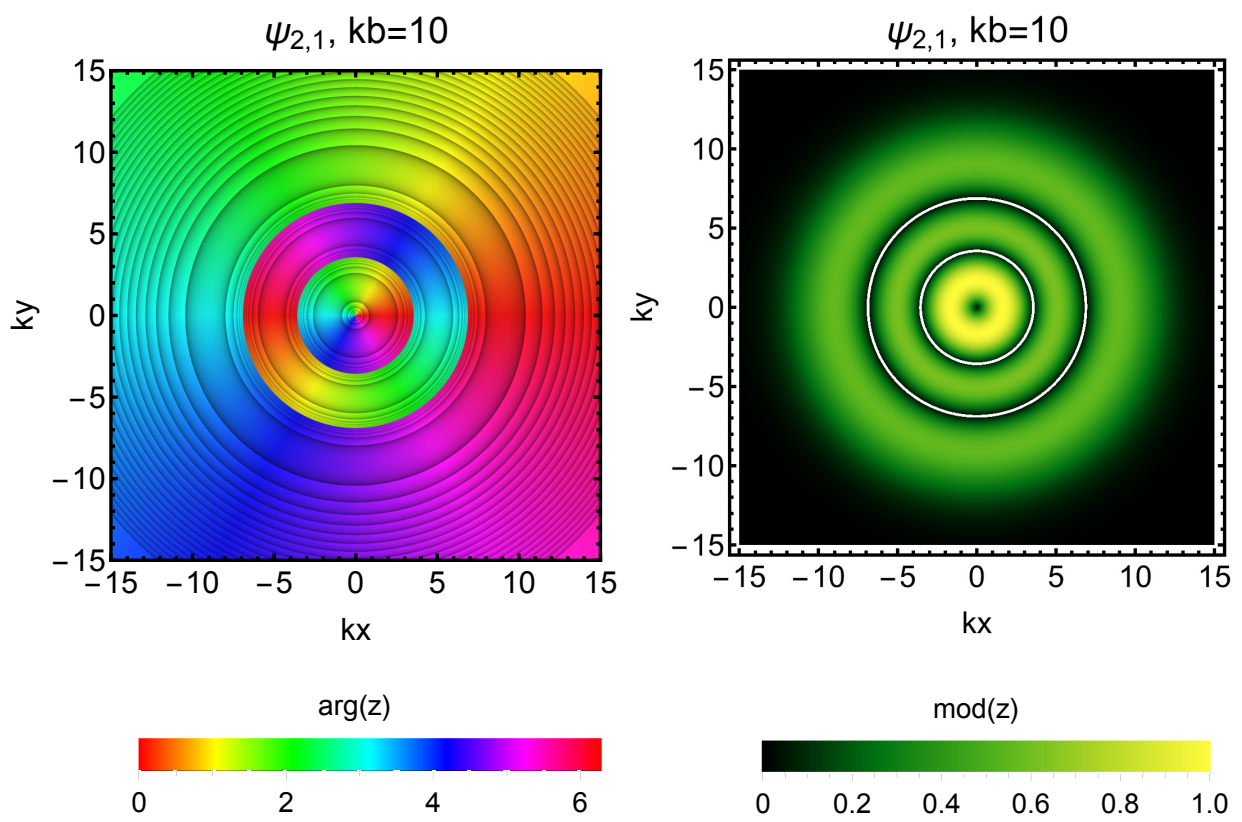

Figure 1.5: Laguerre-Gauss beam focal plane $(k z=0)$ domain colour (left) and intensity (right) plots, with $m=1, n=2$ and $k b=10$. Zero contour is in white for the right hand figure. Note how due to the $e^{i \phi}$ azimuthal dependence the phase makes a complete cycle from 0 to $2 \pi$ around the central zero.

gives the domain colouring of $\psi_{0}$, with the focal plane zeros clearly visible.

In 'Theory of Reflection' [8], Lekner conjectures that these ring zeros are universal for exact, radially finite solutions of the Helmholtz equation. While the proto-beam has all its zeros in the focal plane, the positioning of the zeros can be considerably more complex. For example, consider a Gaussian weight function, as examined by Carter [15], Berry [16] and Karman [17],

$$
\psi_{C}(\rho, z)=\frac{b}{k}\left(e^{\frac{k b}{2}}-1\right)^{-1} \int_{0}^{k} \mathrm{~d} q q \exp \left(i q z+\frac{b q^{2}}{2 k}\right) J_{0}(\kappa \rho) .
$$

For $k b=0$ this reduces to the proto-beam, and has all its zeros in the focal plane. When $k b$ is increased, at certain critical values of $k b$ neighbouring pairs of zeros annihilate, and a new pair is created off plane. This is discussed in greater detail in Chapter 4 


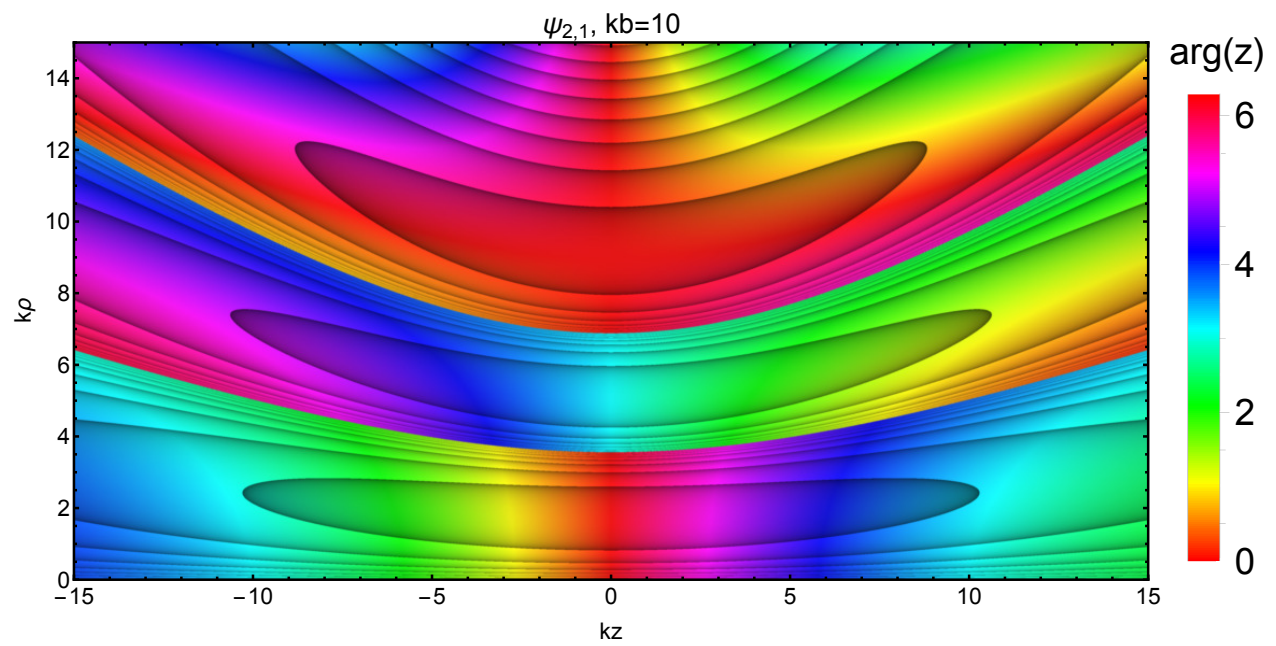

Figure 1.6: Laguerre-gauss beam for $m=1, n=2, k b=10$ in the $x z$ plane, $\phi=0$. Distinctly visible are the sheet zeros, where the phase jumps by $\pi$. The three dimensional picture is obtained by rotating around the beam axis $\rho=0$, with the phase increasing by $\phi$ globally as it is rotated.

In Chapter 4 we include a paper co-authored with John Lekner, produced in the course of this thesis. In it we examine the polarisation properties of electromagnetic beams derived from these wave functions, and show that the beams have polarisation singularities associated with these ring zeros. In particular there is a transition in the polarisation behaviour associated with the zero transition in the Carter wavefunction, $\psi_{C}$.

\subsection{Electromagnetic beams}

So far we have discussed scalar beams, such as particle or acoustic beams. On the other hand, electromagnetic beams are vector beams, which complicates their description. Electromagnetic beams must satisfy Maxwell's equations in free space [18], which in Gaussian units (which we shall be employing throughout), are given by

$$
\begin{aligned}
& \nabla \cdot \boldsymbol{E}=0, \quad \nabla \times \boldsymbol{E}=-\frac{1}{c} \frac{\partial \boldsymbol{B}}{\partial t}, \\
& \nabla \cdot \boldsymbol{B}=0, \quad \nabla \times \boldsymbol{B}=\frac{1}{c} \frac{\partial \boldsymbol{E}}{\partial t} .
\end{aligned}
$$




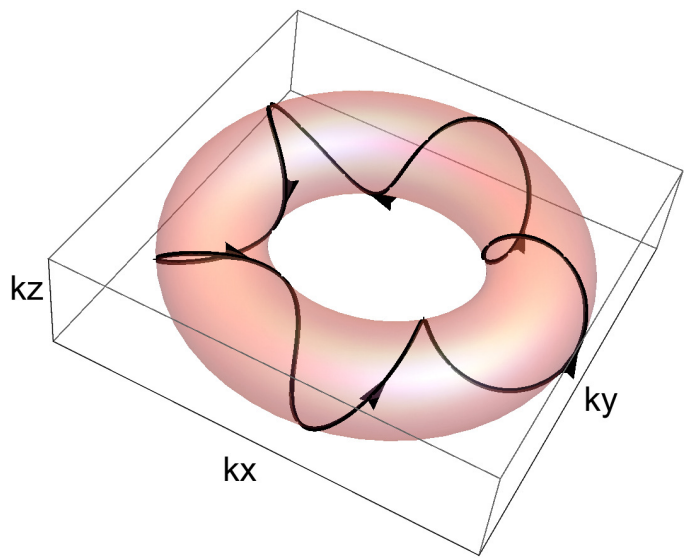

Figure 1.7: Illustration of probability current streamlines (black streamline) around a ring zero (central ring of torus). The number of windings around the toroidal surface is equal to the charge of the zero, in this case 5.

The combination of these implies that each component of $\boldsymbol{E}, \boldsymbol{B}$ must satisfy the wave equation, and thus for monochromatic beams, the Helmholtz equation. Relativistically, rather than a pair of vectors, $\boldsymbol{E}, \boldsymbol{B}$ are components of the antisymmetric electromagnetic tensor. We shall be working in sign convention -+++ , with position four-vectors represented as

$$
x^{\mu}=\left(x^{0}, x^{1}, x^{2}, x^{3}\right)=(c t, x, y, z)
$$

The electromagnetic tensor will therefore be given by

$$
F^{\mu v}=\left(\begin{array}{cccc}
0 & E_{x} & E_{y} & E_{z} \\
-E_{x} & 0 & B_{z} & -B_{x} \\
-E_{y} & -B_{z} & 0 & B_{y} \\
-E_{z} & B_{x} & -B_{y} & 0
\end{array}\right) .
$$

This allows us to write Maxwell's equations compactly as $\partial_{\mu} F^{\mu v}=0$. The electromagnetic tensor itself can be written in terms of a four-potential $A^{\mu}$,

$$
F^{\mu v}=\partial^{\mu} A^{v}-\partial^{v} A^{\mu}
$$




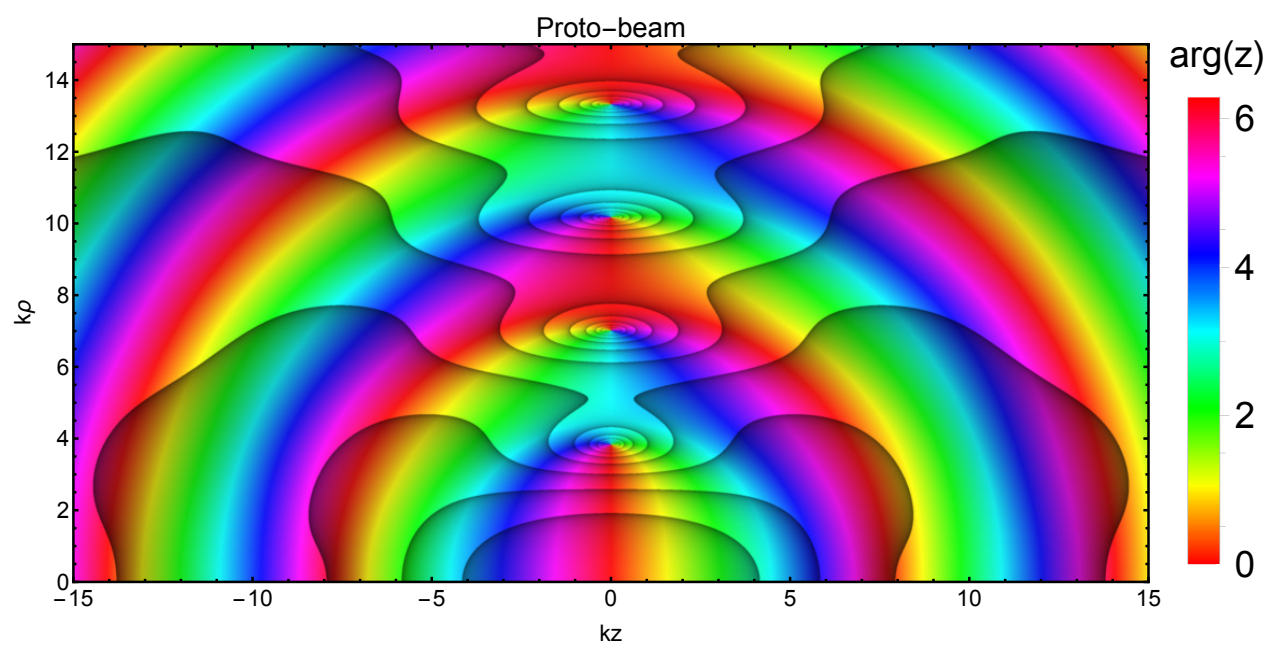

Figure 1.8: Domain colouring of proto-beam in the $k z, k \rho$ plane. The 3D picture is obtained by rotating around the beam axis, $k \rho=0$. Note the zeros in the focal plane $k z=0$, their location being the zeros of $J_{1}(k \rho)$. Around these zeros, the phase circulates, with alternating charge. The origin $k z=0, k \rho=0$ is normalised to unity, thus the second dark contour around the origin gives the contour where the intensity is $e^{-2}$. This is a commonly used measure of the focal extent, by this measure the proto-beam's focal extent is approximately $k|z| \leq 6, k \rho \leq 3$.

In vector notation this gives

$$
\begin{aligned}
& \boldsymbol{E}=-\nabla A^{0}-\frac{1}{c} \frac{\partial \boldsymbol{A}}{\partial t}, \\
& \boldsymbol{B}=\nabla \times \boldsymbol{A} .
\end{aligned}
$$

The electromagnetic tensor is invariant under gauge transformations of the four-potential, allowing us freedom to chose a convenient gauge for the problem at hand. For beams, it is useful to use Lorenz gauge,

$$
\partial_{\mu} A^{\mu}=0
$$

as this implies that the four-potential also satisfies the wave equation. A guaranteed method of generating such a four-potential is to pick $A^{0}=0$, and have 
the spatial components given by the curl of an arbitrary vector that satisfies the wave equation,

$$
\boldsymbol{A}=\nabla \times \Pi, \quad \square \Pi=0 .
$$

Such a four-potential will automatically satisfy the Lorenz gauge condition, due to the divergence of a curl vanishing.

In summary, to generate a beam solution of Maxwell's equations from a solution of the wave equation, take the vector

$$
\Pi=\psi \hat{d},
$$

for some direction $\hat{d}$. Then obtain the vector potential

$$
\boldsymbol{A}=\nabla \times \Pi,
$$

and use this to construct $\boldsymbol{E}, \boldsymbol{B}$. The auxiliary vector $\boldsymbol{\Pi}$ is referred to as a magnetic Hertz potential [19], and in the presence of matter is related to the magnetisation density. We discuss this, including the relativistic formulation, more extensively in Chapter 7 .

This method is extensively employed by Lekner to construct various beams and pulses [8, 20, 21]. Lekner gives four particular directions for $\Pi$ that give convenient properties to the resulting electromagnetic fields. The TE (transverse electric) potential is given by

$$
\boldsymbol{A}_{T E}=\nabla \times \psi \hat{z},
$$

with a wavefunction that has propagation direction along $z$. As the name implies, the electric field (but not magnetic) is transverse to the propagation direction, everywhere. Maxwell's equations are invariant under duality transformations [18], including

$$
\boldsymbol{E} \rightarrow \boldsymbol{B}, \quad \boldsymbol{B} \rightarrow-\boldsymbol{E} .
$$

Taking the dual of the TE fields will give the TM (transverse magnetic) field, with vector potential

$$
\boldsymbol{A}_{T M}=\nabla \times(\nabla \times \psi \hat{z})
$$


The 'LP' and 'CP' fields are so called because in the plane wave limit they give linearly and circularly polarised fields respectively. They are given by

$$
\begin{aligned}
& \boldsymbol{A}_{L P}=\nabla \times \psi \hat{x}, \\
& \boldsymbol{A}_{C P}=\nabla \times \psi(\hat{x}-i \hat{y}) .
\end{aligned}
$$

One can of course substitute $\hat{x} \rightarrow \hat{y}$ to get the other linear polarisation, or $\hat{y} \rightarrow$ $-\hat{y}$ to get the other circular polarisation, for the 'LP' and 'CP' potentials respectively. Lekner shows four non-existence theorems [8]:

1. Pure TEM beams do not exist.

2. Beams of fixed linear polarisation do not exist.

3. Beams of fixed circular polarisation do not exist.

4. Beams or pulses within which the energy velocity is everywhere in the same direction and of magnitude $c$ do not exist.

Note that here TEM refers to a beam which has its electric and magnetic fields perpendicular to the beam axis everywhere. Contrast this with the common Gaussian model, where the electric field is taken to be of fixed linear or circular polarisation, with the magnetic field proportional, and the beam being TEM. In Chapter 2 we shall examine more physical models of electromagnetic beam propagation.

\subsection{Operator algebra}

While operator algebra and Hilbert space theory is often thought of as a technique specific to quantum mechanics, it can be applied to any wave equation. It is a theorem that the space of square integrable function in any number of dimensions, $L^{2}\left(\mathbb{R}^{n}\right)$ is a Hilbert space. In this section we shall give an overview of how standard Hilbert space algebra from quantum mechanics applies generically to $L^{2}$ functions, emphasising that the unique aspect of quantum mechanics is assigning observables to Hermitian operators, and interpreting measured values as eigenvalues of these operators. 
We can represent a square integrable function $\psi: \mathbb{R}^{n} \rightarrow \mathbb{C}$ via a state in a Hilbert space, denoted by the ket $|\psi\rangle$. We can employ the usual notion of the function itself being the projection of its state onto the eigenstate of a 'position operator'. Specifically, we have an operator with non-degenerate spectrum, whose eigenvalues are precisely $\mathbb{R}^{n}$, thus for every point $\boldsymbol{r}$ in $\mathbb{R}^{n}$ there is exactly one ket $|r\rangle$ such that

$$
\hat{R}|\boldsymbol{r}\rangle=\boldsymbol{r}|\boldsymbol{r}\rangle .
$$

The wavefunction at a position $\boldsymbol{x}$ can therefore be interpreted as the projection of the functions ket onto the eigenket of $\boldsymbol{x}$,

$$
\psi(\boldsymbol{r})=\langle\boldsymbol{r} \mid \psi\rangle .
$$

The projection of one position ket onto another will be a delta distribution, as in quantum mechanics, however a choice must be made for the constant prefactor; this decides the Fourier conventions in use. Our conventions will be no factors of $2 \pi$ in the forward (position to wavevector) transform, and $(2 \pi)^{-n}$ for the inverse. This gives

$$
\left\langle\boldsymbol{r}^{\prime} \mid \boldsymbol{r}\right\rangle \equiv \delta^{n}\left(\boldsymbol{r}-\boldsymbol{r}^{\prime}\right)
$$

The choice of Fourier prefactors is equivalent to the choice of prefactors of the inner product of two states; specifically the inner product in position representation is

$$
\langle\phi \mid \psi\rangle=\int_{\mathbb{R}^{n}} \mathrm{~d}^{n} r \phi^{*}(\boldsymbol{r}) \psi(\boldsymbol{r}) .
$$

Any constant commutation algebra is a differential algebra up to a multiplicative factor. Specifically, consider the commutator

$$
\left[x, \frac{\partial}{\partial x}\right] f(x) \equiv x \frac{\partial f}{\partial x}-\frac{\partial x f(x)}{\partial x}=x \frac{\partial f}{\partial x}-f(x)-x \frac{\partial f}{\partial x}=f(x) .
$$

We can see that this gives the commutator

$$
\left[x, \frac{\partial}{\partial x}\right]=1 .
$$


Since our functional space is differentiable, we can therefore introduce a constant commutation algebra via the differential algebra. Define a 'wavevector' operator $\hat{k}$ via

$$
\left[\hat{X}^{i}, \hat{k}^{j}\right]=-i \delta^{i j} .
$$

In position representation we can see that this will be given by $\hat{k}_{i}=-i \frac{\partial}{\partial x^{i}}$. It can easily be shown that the position representation of the eigenstates of $\hat{k}$ are plane waves,

$$
\langle\boldsymbol{r} \mid \boldsymbol{k}\rangle=e^{i \boldsymbol{k} \cdot \boldsymbol{r}} .
$$

Our Fourier conventions imply that the projection of a wavevector eigenket onto another is given by

$$
\left\langle\boldsymbol{k}^{\prime} \mid \boldsymbol{k}\right\rangle=(2 \pi)^{n} \delta^{n}\left(\boldsymbol{k}-\boldsymbol{k}^{\prime}\right) .
$$

The Fourier transform of a function $\psi(\boldsymbol{r})$ is given by

$$
\tilde{\psi}(\boldsymbol{k})=\int_{\mathbb{R}^{n}} \mathrm{~d}^{n} r e^{-i \boldsymbol{k} \cdot \boldsymbol{r}} \psi(\boldsymbol{r}) .
$$

Rewritten in Dirac notation this is

$$
\tilde{\psi}(\boldsymbol{k})=\int_{\mathbb{R}^{n}} \mathrm{~d}^{n} r\langle\boldsymbol{k} \mid \boldsymbol{r}\rangle\langle\boldsymbol{r} \mid \psi\rangle=\langle\boldsymbol{k} \mid \psi\rangle,
$$

which illustrates that the Fourier transform is nothing more than the projection of the state in wavevector representation. In non-relativistic quantum mechanics one has a physical interpretation, namely that $|\psi(\boldsymbol{r})|^{2}$ is the probability density of measuring a particle at position $\boldsymbol{r}$ (to find an actual probability one needs to integrate over some finite volume). Outside of quantum mechanics, the physical interpretation will be specific to the theory involved. For example, if the wave under consideration is an acoustic wave, the real (or imaginary) part of the wavefunction $\psi(\boldsymbol{r})$ will describe the pressure at the point $\boldsymbol{r}$ directly. For electromagnetic waves, rather than the Hilbert space of $L^{2}$ scalar fields, we have $L^{2}$ tensor fields. These can be constructed via the Kronecker product of the scalar Hilbert space. For example, the $\mu^{t h}$ component of the four-potential, $A^{\mu}(\boldsymbol{r})$ can be taken to be the projection of the state of $A$,

$$
\langle\boldsymbol{r}, \mu \mid A\rangle
$$


with the position eigenkets for four-vectors satisfying

$$
\left\langle\boldsymbol{r}^{\prime}, \mu \mid \boldsymbol{r}, v\right\rangle=\delta^{n}\left(\boldsymbol{r}-\boldsymbol{r}^{\prime}\right) \delta^{\mu \nu}
$$

For example, a wavevector eigenket projected onto the position eigenket with index $\mu$ is given by

$$
\langle\boldsymbol{r}, \mu \mid \boldsymbol{k}\rangle=\hat{e}^{\mu} e^{i \boldsymbol{k} \cdot \boldsymbol{r}},
$$

with $\hat{e}^{\mu}$ the $\mu^{t h}$ basis vector for the tangent space at $\boldsymbol{r}$.

Finally, an 'angular wavevector operator' can be defined,

$$
\hat{l}=\hat{r} \times \hat{k} \text {. }
$$

In quantum mechanics this is of course proportional to the angular momentum operator. Bessel beams are simultaneous eigenfunctions of the $z$ component of $\hat{l}$ and $\hat{k}$,

$$
\hat{l}_{z}=-i \frac{\partial}{\partial \phi}, \quad \hat{k}_{z}=-i \frac{\partial}{\partial z}
$$

The eigenvalues for a Bessel beams

$$
e^{i(q z+m \phi)} J_{m}(\kappa \rho)
$$

are $m$ and $q$ for $\hat{l}_{z}$ and $\hat{k}_{z}$ respectively.

\subsection{Academic contributions}

With the exception of chapters 3 and 4 , all chapters are my own independent research, with helpful feedback from my supervisor, John Lekner. Chapters 3 and 4 reproduce verbatim two papers that I have co-authored with John Lekner during the course of this thesis. These have been published in Optics Communications [22] and Journal of Optics [23] respectively. 


\section{Chapter 2}

\section{Focusing limits of finite beams}

\subsection{Introduction}

In this chapter we shall consider the properties of finite beams, with the emphasis being on investigating how tightly a beam can be focused. It is our view that spot size and focal extent should be distinguished. Spot size in our view describes the size of the central maximum, whereas focal extent should describe how concentrated the intensity is to the focal point. Commonly, measuring the focal extent is done through spot size for singly peaked beams, and the second moment based 'beam quality' measure for more complicated beam forms. We will show that spot size does not measure focal extent adequately for non-paraxial, tightly focused beams, and that beam quality is divergent for non-paraxial beams. Instead of these, we define two novel focal measures, one for single peaked beams, and one general, and use these to demonstrate that the proto-beam, as investigated by Lekner [14], is in fact the unique tightest focused scalar beam.

We shall begin however by describing the basic properties of finite beams, including their invariants. Labelling the propagation direction $z$, the finiteness condition we shall require is radial square integrability,

$$
\forall z \iint_{-\infty}^{\infty} \mathrm{d} x \mathrm{~d} y|\psi(x, y, z)|^{2}<\infty
$$


This is a sufficient criterion for the beam being decomposable as a superposition of Bessel beams. As we have discussed in the introduction, Bessel beams are the simultaneous eigenfunctions of the $\hat{l}_{z}$ and $k_{z}$ operators,

$$
\hat{l}_{z}=-i \frac{\partial}{\partial \phi}, \quad \hat{k}_{z}=-i \frac{\partial}{\partial z}
$$

For quantum mechanical beams, $\hbar \hat{l}_{z}$ corresponds to the $z$ angular momentum operator, and $\hbar k_{z}$ to the momentum operator. Bessel beams form a basis for radially finite beams, with an arbitrary beam being given by

$$
\psi(\rho, \phi, z)=\sum_{m=-\infty}^{\infty} e^{i m \phi} \int_{0}^{\infty} \mathrm{d} \kappa \kappa f_{m}(\kappa) e^{i q(\kappa) z} J_{m}(\kappa \rho) .
$$

The $z$ component of the wave-number $q(\kappa)$ is decided by the particular wave equation under consideration. For the Schrödinger equation, and the wave equation, the spatial dependence is given by the Helmholtz equation, so the relation is $q(\kappa)=\sqrt{k^{2}-\kappa^{2}}$, with $k$ the magnitude of the wave-number. For paraxial waves the relation is instead $q(\kappa)=k-\frac{\kappa^{2}}{2 k}$. Note that a Taylor expansion up to order $\kappa^{2}$ gives

$$
\sqrt{k^{2}-\kappa^{2}}=k-\frac{\kappa^{2}}{2 k}+\mathscr{O}\left(\kappa^{4}\right) .
$$

It is important that the wave does not have exponential growth in $z$; this restricts the range of $\kappa$ for the Helmholtz equation to $\kappa \in[0, k]$. For the paraxial equation $\kappa$ is unrestricted. As we shall see, this has implications for the maximal focusing of the beam.

Note that in this chapter we shall make numerous references to the 'intensity' of beams. For scalar beams, this should be interpreted to be the modulus squared, and for electromagnetic beams, the energy density.

\subsection{Beam invariants}

In general, solutions to a wave equation will have certain conservation laws. For example, Schrödinger's equation has the conservation of probability: the 
probability density is given by $\varrho=|\psi|^{2}$, and the probability current is given by $\boldsymbol{j}=\frac{\hbar}{m} \operatorname{Im}\left(\psi^{*} \nabla \psi\right)$, satisfying the conservation law

$$
\frac{\partial \varrho}{\partial t}+\nabla \cdot \boldsymbol{j}=0
$$

For electromagnetic waves, the energy density is given by

$$
\frac{1}{8 \pi}\left(E^{2}+B^{2}\right)
$$

and the momentum density by

$$
\boldsymbol{S}=\frac{1}{4 \pi} \boldsymbol{E} \times \boldsymbol{B},
$$

with the free-space conservation law given by Poynting's theorem

$$
\frac{\partial u}{\partial t}+\nabla \cdot \boldsymbol{S}=0
$$

Other conservation laws are related to tensor quantities, such as the Maxwell stress tensor, angular momentum, etc. It can be shown [24,25] that these types of conservation laws applied to beams give rise to beam invariants, quantities which are invariant for a given radial slice of the beam. Specifically, given a density $\varphi(\boldsymbol{r})$, and labelling the propagation direction $\hat{z}$, a beam invariant is a quantity such that

$$
\partial_{z} \int \mathrm{d}^{2} r \varphi(x, y, z)=0
$$

where we have integrated over a plane of constant $z$. In other words, a beam invariant gives a quantity per unit length that is conserved through beam propagation.

One quantity that is not universally an invariant is the probability density per unit length of a quantum mechanical beam. In general, we do not have

$$
\partial_{z} \int \mathrm{d}^{2} r|\psi|^{2}=0
$$

The probability density for superpositions of Bessel beams is given by

$$
\begin{aligned}
|\psi|^{2}=\sum_{m, m^{\prime}=-\infty}^{\infty} e^{i\left(m-m^{\prime}\right) \phi} \iint_{0}^{\pi / 2} & \sin \alpha \mathrm{d} \alpha e^{i k\left(\cos \alpha-\cos \alpha^{\prime}\right) z} \\
& \times J_{m}(k \sin \alpha \rho) J_{m}\left(k \sin \alpha^{\prime} \rho\right) f_{m}^{*}\left(\alpha^{\prime}\right) f_{m}(\alpha) .
\end{aligned}
$$


Two relevant orthogonality integrals are

$$
\begin{gathered}
\int_{0}^{2 \pi} \mathrm{d} \phi e^{i\left(m-m^{\prime}\right) \phi}=2 \pi \delta_{m, m^{\prime}}, \\
\int_{0}^{\infty} \rho \mathrm{d} \rho J_{m}(k \sin \alpha) J_{m}\left(k \sin \alpha^{\prime} \rho\right)=\frac{\delta\left(k \sin \alpha-k \sin \alpha^{\prime}\right)}{k \sin \alpha}=\frac{\delta\left(\alpha-\alpha^{\prime}\right)}{k^{2} \sin \alpha \cos \alpha} .
\end{gathered}
$$

with the above equation assuming $\alpha, \alpha^{\prime} \in[0, \pi / 2]$. We can see that this orthogonality reduces the radial integral of $|\psi|^{2}$ to

$$
\int \mathrm{d}^{2} r|\psi|^{2}=\frac{2 \pi}{k^{2}} \sum_{m=-\infty}^{\infty} \int_{0}^{\pi / 2} \mathrm{~d} \alpha \tan \alpha\left|f_{m}(\alpha)\right|^{2}
$$

which is clearly independent of $z$, and is hence a beam invariant. The assumption that $\alpha \in[0, \pi / 2]$ is crucial: it ensures that all the components are propagating along $z$, and allows to make the transition from $\delta\left(\sin \alpha-\sin \alpha^{\prime}\right)$ to $\delta\left(\alpha-\alpha^{\prime}\right)$. If we allow backward propagating components, we can include them by either extending the range to $\alpha \in[0, \pi]$, or by adding terms with an $e^{-i k \cos \alpha z}$ dependence to the integrals. We shall do the latter as it is simpler to manipulate the $\delta$ distributions this way. In this case the beam will be

$$
\begin{aligned}
\psi(\rho, \phi, z)=\sum_{m=-\infty}^{\infty} e^{i m \phi} & \int_{0}^{\pi / 2} \sin \alpha \mathrm{d} \alpha \\
& \times\left(f_{m}(\alpha) e^{i k \cos \alpha z}+g_{m}(\alpha) e^{-i k \cos \alpha z}\right) J_{m}(\sin \alpha \rho) .
\end{aligned}
$$

The radial integral of $|\psi|^{2}$ then gives

$$
\begin{aligned}
\sum_{m=-\infty}^{\infty} \int_{0}^{\pi / 2} \mathrm{~d} \alpha \tan \alpha\left(\left|f_{m}(\alpha)\right|^{2}+\left|g_{m}(\alpha)\right|^{2}+\right. & e^{2 i k \cos \alpha z} f_{m}^{*}(\alpha) g_{m}(\alpha) \\
& \left.+e^{-2 i k \cos \alpha z} f_{m}(\alpha) g_{m}^{*}(\alpha)\right) .
\end{aligned}
$$

Thus, if we allow backward propagating components the interference between the forward and backward propagation results in a $z$ dependence of the probability density per unit length. As an example, consider the superposition of beams with $g_{0}=f_{0}=\cos \alpha, g_{m}=f_{m}=0$ otherwise. The probability density per unit length, up to a normalisation, will then be given by

$$
\int_{0}^{\pi / 2} \mathrm{~d} \alpha 2 \sin \alpha \cos \alpha(1+\cos (2 k z \cos \alpha))=1+\frac{\sin k z(2 k z \cos k z-\sin k z)}{(k z)^{2}} .
$$


Figure 2.1 gives a plot of this function. We can see that it oscillates around a constant value, with a decaying amplitude, with an oscillation wavelength of $\frac{2 \pi}{k}$. As a result, although in a certain sense the 'average' probability density per unit length is constant, the actual value oscillates with $z$.

We can define an averaging process to be

$$
\langle f(z)\rangle=\lim _{\Delta L \rightarrow 0} \frac{1}{\Delta L} \int_{L_{0}}^{L_{0}+\Delta L} \mathrm{~d} z f(z) .
$$

The interference terms for solutions of the Helmholtz equation are given by integrals of the form

$$
\int_{0}^{\pi / 2} \cos (2 k z \cos \alpha) f(\alpha) \mathrm{d} \alpha
$$

The average of a cosine gives

$$
\langle\cos (q z)\rangle=\lim _{\Delta L \rightarrow 0} \frac{1}{\Delta L} \int_{L_{0}}^{L_{0}+\Delta L} \mathrm{~d} z \cos q z=\frac{\sin \left(q L_{0}+q \Delta L\right)-\sin \left(q L_{0}\right)}{q \Delta L}=0 .
$$

It is trivial that the average of a constant is itself. As a result, if we average the probability density per unit length over a sufficiently large length scale, it will be equal to the sum of the forward and backward propagating probability densities per unit length.

For paraxial beams, any solution will have $|\psi|^{2}$ as a beam invariant: we can write

$$
\psi=\sum_{m=-\infty}^{\infty} e^{i m \phi} \int_{-\infty}^{k} \mathrm{~d} q e^{i q z} J_{m}(\kappa(q) \rho) f_{m}(k, q), \quad \kappa(q)=\sqrt{2 k(k-q)} .
$$

The radial integral for each $m, m^{\prime}$ gives

$$
\frac{1}{\kappa(q)} \delta\left(\kappa(q)-\kappa\left(q^{\prime}\right)\right)=\frac{1}{k} \delta\left(q-q^{\prime}\right),
$$

where we have used the Dirac delta composition identity, and the fact that since $\kappa(q)$ is invertible, there will be only one root. This ensures that the plane wave terms in $z$ cancel, and the probability density per unit length is given by

$$
\sum_{m=-\infty}^{\infty} \int_{-\infty}^{k} \mathrm{~d} q\left|f_{m}(k, q)\right|^{2}
$$




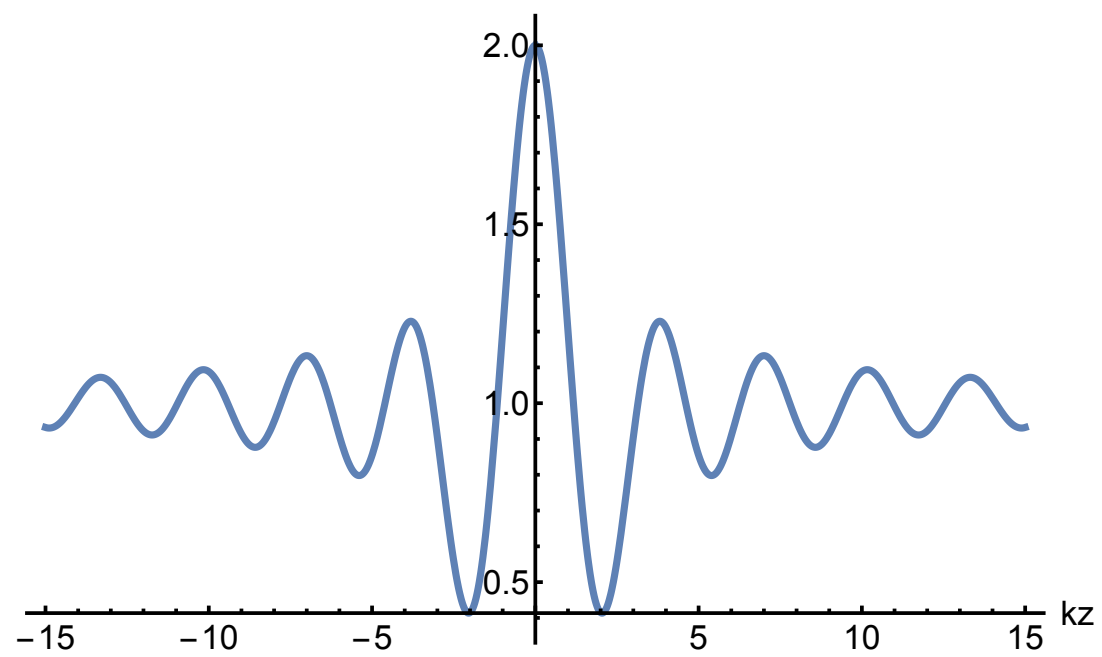

Figure 2.1: Probability per unit length from equation 2.2.12). We can see that it is oscillatory about $A_{0}=1$, with a decaying amplitude

\subsection{Focusing of beams}

One fundamental question we wish to explore is: how tightly can a beam be focused? To answer this, we must first consider the various measures of focal extent. We will be restricting our attention to systems with azimuthal symmetry, as the focal extent of a hollow beam can be difficult to define. To a first approximation, the radial intensity profile of a beam can be taken to be bellshaped, with this model being exact for a paraxial Gaussian beam.

The functional form of the Gaussian is

$$
\psi_{G}(\rho, z)=\frac{b}{b+i z} \exp \left(i k z-\frac{k \rho^{2}}{2(b+i z)}\right)
$$

a solution of the paraxial Helmholtz equation. The key parameter, $b$ determines how tightly the beam is focused, and is often referred to as the 'Rayleigh range', $z_{R}$, the distance from the focal plane where the 'beam radius' is $\sqrt{2}$ of that at $z=0$. The 'beam radius' is given by

$$
w(z)=\sqrt{\frac{2}{k b}\left(b^{2}+z^{2}\right)},
$$


and is defined as the radius where the intensity drops off to $e^{-2}$ of that on the beam axis. The beam radius at the origin is referred to as the 'beam waist', and is given by $w_{0}=\sqrt{2 b / k}$. The phase of the term $b+i z$ is referred to as the 'Gouy phase', $\Phi(z)=\arctan (z / b)$. Finally, the radius of curvature of the wavefronts is given by $R(z)=z\left(1+(b / z)^{-2}\right)$. There is no restriction to how tightly a Gaussian beam wavefunction can be focused, as one can send $b$ arbitrarily close to zero to get a tighter focus. Physical beams however are governed by the exact Helmholtz equation, with paraxial beams only an approximation. In Chapter 3. we show the paraxial approximation is only valid for $b^{2}+z^{2} \gg \rho^{2}$ and $k^{2}\left(b^{2}+z^{2}\right) \gg 1$. We can see that in the limit of small $b$, the approximation is valid only close to the beam axis, far from the focal plane. Physical beams are limited in how tightly they can be focused, and to investigate these limits we must use exact solutions of the Helmholtz equation.

In general, for bell shaped intensity profiles, one uses the decay rates to measure the focal extent. Specifically, measures such as 'full width at half maximum' (FWHM), or the Gaussian beam radius $\left(e^{-2}\right)$ measure. For FWHM, the beam radius is simply defined to be the value of $\rho$ such that the intensity is half of the peak. For $e^{-2}$, it is the radius such that intensity is $e^{-2}$ of the peak. This is an adequate measure for uniformly decaying beams, that decay sufficiently rapidly. Indeed, if the decay rate is of order $e^{-\rho^{2}}$, most of the intensity of the beam will be contained within $e^{-2}$ of peak. As we shall see shortly, for a Gaussian beam, in the focal plane roughly $86 \%$ of the total intensity is contained within the $e^{-2}$ radius.

However, for exact solutions of the Helmholtz equation, the decay rates can be much slower, for example the proto-beam has a radial wavefunction proportional to $J_{1}(k \rho) / k \rho$, which has a decay rate of $\rho^{-3 / 2}$, and thus an intensity decay rate of $\rho^{-3}$. In this case, only $76.6 \%$ of the intensity is contained within the $e^{-2}$ radius. We can see this through numerical evaluation. The total intensity can be calculated symbolically, and comes out to be $\frac{2 \pi A^{2}}{2 k^{2}}$, where $A$ is the prefactor of the wavefunction. The intensity at the origin is $A^{2} / 4$. We can solve 
numerically for $k \rho$ such that

$$
J_{1}(k \rho)=\frac{1}{2 e} k \rho .
$$

This comes out to be $k \rho \approx 2.584$. The ratio of the integral up to this radius over the integral over all space can be evaluated numerically, and comes out to approximately 0.766 . Thus, almost a quarter of the intensity is outside this radius. The biggest issue with this measure is that it is not symbolically tractable in general. This is fine experimentally, or when considering a specific beam, as one can use numerics in this case. However, our aim is to be as general as possible, and as such we want a measure that can be symbolically evaluated.

A further issue is that the $e^{-2}$ measure is not always consistent with integral based measures. In this section, we will define a parameter $\eta$ that is the ratio of the radial integral of intensity, to the peak intensity. For centrally peaked beams this is an unambiguous measure of focal tightness: the tighter the focus, the lower the ratio. We will show that the proto-beam is the tightest focused beam under this measure. On the other hand, we can numerically evaluate the $e^{-2}$ radius for beams with $q^{k b}$ weight functions to see what trend it has. Figure 2.2(a) gives this plot. We can see that it is monotonically increasing with $k b$, which could mislead one into thinking a beam with $k b<1$ is tighter focused than the proto-beam. To see how this issue arises, Figure 2.2(b) gives a plot of the intensity contained in the $e^{-2}$ radius as a fraction of total intensity. It is clear that for small $k b$ this fraction drastically decreases, and little of the total intensity is contained in the first 'spot'. This is because tightly focused beams are highly oscillatory in their radial weight function.

An alternative method is to use an integral based measure such as the 1$e^{-2}$, or $86 \%$ measure. This measure is simply defined to be the radius such that a fraction of $1-e^{-2} \approx 0.86$ of the total intensity is contained within. Specifically, it is the radius $\rho_{0}$ such that

$$
\frac{\int_{0}^{\rho_{0}} \rho \mathrm{d} \rho|\psi(\rho)|^{2}}{\int_{0}^{\infty} \rho \mathrm{d} \rho|\psi(\rho)|^{2}}=1-e^{-2} \approx 0.86 .
$$

For a Gaussian beam, this is equivalent to the $e^{-2}$ measure. However as we 


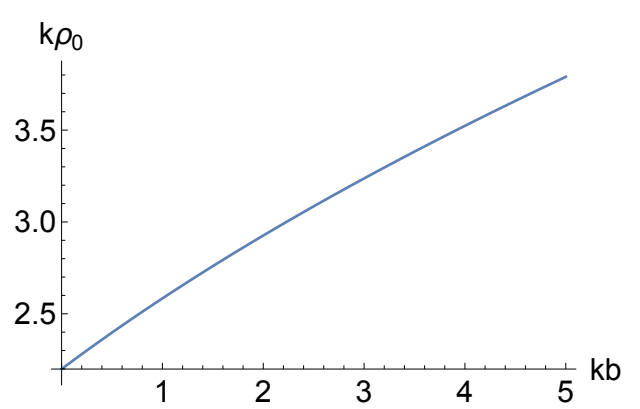

(a)

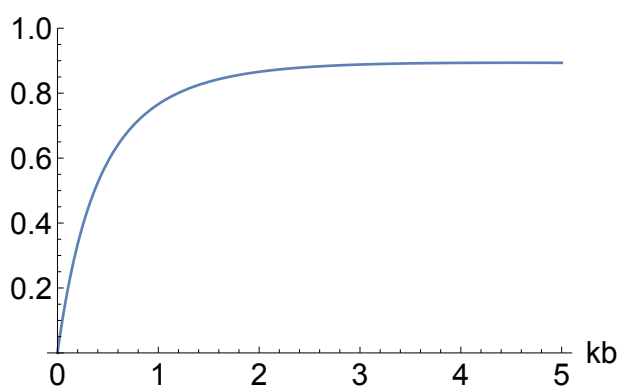

(b)

Figure 2.2: $e^{-2}$ radius, left, and fraction of total intensity contained in $e^{-2}$ radius for $q^{k b}$ beams. Although the 'spot' size decreases monotonically with decreasing $k b$, the fraction of intensity does as well. Below $k b \approx 1$ the fraction drops significantly, in this regime the $e^{-2}$ spot size is a poor focal measure.

have seen with the proto-beam, it will in fact be larger than the $e^{-2}$ measure for exact solutions. However, in general the $86 \%$ radius will not have a closed form expression, being more often used for experimental analysis. There are other related measures, such as moment measures [see 26], that compare integrals over all space with different moment weightings. These can be problematic for exact solutions of the Helmholtz equation; a Gaussian falls off faster than any polynomial in $r$, which guarantees the convergence of such a focal measure. On the other hand, generalised Bessel beams can have $\rho^{n}$ falloff. For example, a 'first moment' integral can be defined to be

$$
\int_{\mathbb{R}^{2}} \mathrm{~d}^{2} r \rho|\psi(\rho, \phi)|^{2}
$$

For the proto-beam, even though the beam has finite invariants, this 'first' moment integral is divergent.

We shall use a simple measure, the ratio total intensity to the peak intensity, as this method will allow us to obtain closed form symbolic expressions. While it may not be as directly relevant to physical 'spot size', our aim is to obtain the tightest focused beam, and our choice of measure will be sufficient to determine this. 


\subsection{Axial focal extent}

We shall first examine the extent of axial focusing, i.e. how rapidly the amplitude decays from its maximal value, along the beam axis. It will be convenient to rewrite 2.1.3 into an integral of $z$ wave-number, $q$, and assume without loss of generality that the peak amplitude of the beam occurs at $\rho=0$, which we shall refer to as the beam axis. We will also restrict our attention to beams that areforward propagating far from the focus (near zeros the beam can be locally backward propagating due to the phase circulation). This allows us to write the wavefunction as

$$
\psi(\rho, \phi, z)=\sum_{m=-\infty}^{\infty} e^{i m \phi} \int_{0}^{k} \mathrm{~d} q f_{m}(q) e^{i q z} J_{0}(\kappa \rho), \quad \kappa^{2}+q^{2}=k^{2} .
$$

The wavefunction on the beam axis is therefore given by the Fourier transform of its $m=0$ weight function, $f_{0}(q)$, as all of the higher order Bessel functions are zero at $\rho=0$.

Plancharel's theorem shows that the $L^{2}$ norm of a function is equal to the $L^{2}$ norm of its Fourier transform,

$$
\int_{-\infty}^{\infty} \mathrm{d} z|\psi(z)|^{2}=\int_{-\infty}^{\infty} \frac{\mathrm{d} q}{2 \pi}|\tilde{\psi}(q)|^{2}
$$

where $\tilde{\psi}$ is the Fourier transform of $\psi$,

$$
\tilde{\psi}(q)=\int_{-\infty}^{\infty} \mathrm{d} z \psi(z) e^{-i q z}
$$

Note that if $\psi$ is a wavefunction, it is dimensionless: this gives $\tilde{\psi}$ the dimensionality of length, i.e $\tilde{\psi}$ is a density in Fourier space. Furthermore, using the definition in (2.4.1), we have

$$
f_{0}(q)=2 \pi \tilde{\psi}(q)=2 \pi \int_{-\infty}^{\infty} \mathrm{d} z e^{-i q z} \psi(0, z) .
$$

In terms of the weight function the $L^{2}$ norm on the beam axis is given by

$$
\int_{-\infty}^{\infty} \mathrm{d} z|\psi(0, z)|^{2}=2 \pi \int_{0}^{k} \mathrm{~d} q f_{0}(q)
$$


For finite beams, we first have the constraint of radial boundedness, by which we mean the function must be radially square integrable. In terms of the weight function, the radial square integral is given by

$$
2 \pi \sum_{m=-\infty}^{\infty} \int_{0}^{k} \mathrm{~d} q q^{-1}\left|f_{m}(q)\right|^{2}
$$

This constrains the valid choices of weight function. We have without loss of generality picked our coordinate system such that the origin is at the maximum, and we can normalise so that the maximum amplitude is one.

$$
\left.|\psi|^{2}\right|_{z=0}=1,\left.\quad \frac{\partial|\psi|^{2}}{\partial z}\right|_{z=0}=0
$$

If we write the $m=0$ weight function as $f_{0}(q)=a(q)+i b(q)$ for real functions $a, b$, the wave-function at the origin is given by

$$
\psi_{0}=\int_{0}^{k} \mathrm{~d} q a(q)+i b(q)=e^{i \alpha}
$$

for some $\alpha$. As this scheme normalises all beams to be of unit amplitude at the origin, more tightly focused beams will therefore have a smaller $L^{2}$ norm. Thus, to find the limit of tightest focus, we must find the weight function in the range $q \in[0, k]$ that minimises the $L^{2}$ norm, subject to the radial boundedness constraint.

The derivative of the modulus is given by

$$
\frac{\partial|\psi|^{2}}{\partial z}=\operatorname{Re}\left(\psi^{*} \frac{\partial \psi}{\partial z}\right) .
$$

The value of this at the origin can be written as

$$
\int_{0}^{k} \mathrm{~d} q q(b(q) \cos \alpha-a(q) \sin \alpha)
$$

For this to be a zero, a necessary condition is

$$
\frac{\int_{0}^{k} \mathrm{~d} q q b(q)}{\int_{0}^{k} \mathrm{~d} q q a(q)}=\tan \alpha .
$$


This is equivalent to $\psi$ and its $z$ derivative having a phase difference of ( $n+$ $1 / 2$ ) $\pi$ for some integer $n$, which can also be ascertained from equation (2.4.9). This will always be true for weight functions $f(q)$ that have a phase independent of $q$. Such weight functions lead to amplitudes that are symmetric under $z \rightarrow-z$, and hence such beams will always have a vanishing derivative at the origin, and will always have a local maximum there.

The Cauchy-Schwartz inequality for square integrable functions on the interval $[0, k]$ is

$$
\left|\int_{0}^{k} \mathrm{~d} q F(q) G^{*}(q)\right|^{2} \leq \int_{0}^{k} \mathrm{~d} q|F(q)|^{2} \times \int_{0}^{k} \mathrm{~d} q|G(q)|^{2} .
$$

To compare the value of the integral over the entire beam axisto the value at the origin, we set $F(q)=f_{0}(q), G(q)=1$. This gives us

$$
\left|\int_{0}^{k} \mathrm{~d} q f_{0}(q)\right|^{2} \leq k \int_{0}^{k} \mathrm{~d} q\left|f_{0}(q)\right|^{2} .
$$

This gives us a measure of axial focal concentration for azimuthally symmetric beams: for a given beam $\psi$, with a global amplitude maximum at $z_{0}$, define the length $\chi$ to be

$$
\chi=\frac{1}{\left|\psi\left(z_{0}\right)\right|^{2}} \int_{-\infty}^{\infty} \mathrm{d} z|\psi(z)|^{2} \geq 2 \pi k^{-1} \equiv \lambda,
$$

where $\lambda$ is the usual definition of wavelength. If a dimensionless parameter is desired, $\chi / \lambda$ is a natural choice. $\chi$ will measure the tightness of the axial focus, with a more focused beam having $\chi$ closer to $\lambda$. In terms of arbitrary weight functions, the measure is

$$
\chi=\frac{2 \pi \int_{0}^{k} \mathrm{~d} q\left|f_{0}(q)\right|^{2}}{\left|\int_{0}^{k} \mathrm{~d} q f_{0}(q) e^{i q z_{0}}\right|^{2}} .
$$

The Cauchy-Schwartz inequality reaches equality iff. $F$ and $G$ are scalar multiples. This implies the tightest axial focus is for the beam with $f_{0}(q)$ constant. This is not physical, as such a beam will not be square integrable. However one can achieve an axial focus arbitrarily close to this. To illustrate this, consider the 
weight function

$$
f_{m}(k, q)=\left\{\begin{array}{l}
(q / k)^{k b}, \quad m=0, \\
0, \quad \text { otherwise. }
\end{array}\right.
$$

The amplitude at the origin is $k^{2}(k b+1)^{-2}$, and the integral over all space is $2 \pi k(2 b+1)^{-1} \cdot \chi$ is therefore given by

$$
\chi=\frac{2 \pi}{k} \frac{(k b+1)^{2}}{2 k b+1}
$$

which attains its minimal value of $\lambda=2 \pi k^{-1}$ when $b=0$. However, the radial integral gives

$$
\frac{2 \pi}{k^{2 k b}} \int_{0}^{k} \mathrm{~d} q q^{2 k b-1}=\frac{1}{2 k b},
$$

which diverges at $k b=0$, but converges for $k b>0$. Thus, this family of beams can be focussed arbitrarily close to the maximal limit, but never reach it. If we allow backward propagation, the focus can be tightened, but only up to $\chi=\lambda / 2$. To see this, consider a general function, with a global maximum at the origin, and a compactly supported Fourier transform,

$$
\psi(z)=\int_{\zeta}^{\zeta+\Delta \zeta} \frac{\mathrm{d} q}{2 \pi} e^{i q z} \tilde{\psi}(q)
$$

The $\chi$ parameter for this function will be given by

$$
\chi=\frac{\int_{\zeta}^{\zeta+\Delta \zeta} \frac{\mathrm{d} q}{2 \pi}|\tilde{\psi}(q)|^{2}}{\left|\int_{\zeta}^{\zeta+\Delta \zeta} \frac{\mathrm{d} q}{2 \pi} \tilde{\psi}(q)\right|^{2}}
$$

The Cauchy-Schwartz inequality for this case is

$$
\left|\int_{\zeta}^{\zeta+\Delta \zeta} \mathrm{d} q \tilde{\psi}(q)\right|^{2} \leq \Delta \zeta \int_{\zeta}^{\zeta+\Delta \zeta} \mathrm{d} q|\tilde{\psi}(q)|^{2} .
$$

As a result, we have $\chi \geq 2 \pi(\Delta \zeta)^{-1}$. Thus, the maximal focussing is inversely proportional to the size of the interval of allowed values for $q$; larger intervals allow for tighter focusing. This is consistent with what we have seen for the Gaussian beam: the allowed values are $q \in(-\infty, k]$, so we can focus arbitrarily 
tightly. We shall note that since the Gaussian beam in 2.3.1] is normalised to unity at the origin, its $\chi$ parameter is given by

$$
\int_{-\infty}^{\infty} \frac{b^{2}}{b^{2}+z^{2}} \mathrm{~d} z=\pi b
$$

We can see that for a Gaussian beam, the $\chi$ parameter is proportional to its Rayleigh range.

\subsection{Radial focusing}

For the radial focusing, we will use a similar measure. The measure will be the radial integral of the modulus, divided by the modulus at the maximum

$$
\eta=\frac{\int_{0}^{\infty} \int_{-\pi}^{\pi} \mathrm{d} \rho \mathrm{d} \phi \rho|\psi(\rho, \phi, 0)|^{2}}{\left|\psi_{0}\right|^{2}}
$$

where $\psi_{0}$ is the maximum modulus. Here we have assumed the maximum is yet again at $\rho=0 . \eta$ has the dimensions of area. In terms of the weight function, the integral over all space is given by

$$
2 \pi \sum_{m=-\infty}^{\infty} \int_{0}^{k} \mathrm{~d} q \frac{\left|f_{m}(k, q)\right|^{2}}{q} .
$$

The value at the origin is the same as before. We must find a relevant CauchySchwartz inequality for this measure. This can be achieved by taking (2.4.12, and setting $F(q)=\frac{f_{0}(q)}{\sqrt{q}}, G(q)=\sqrt{q}$. This will give

$$
\left|\int_{0}^{k} \mathrm{~d} q f_{0}(q)\right|^{2} \leq \int_{0}^{k} \mathrm{~d} q \frac{\left|f_{0}(q)\right|^{2}}{q} \int_{0}^{k} \mathrm{~d} q q=\frac{k^{2}}{2} \int_{0}^{k} \mathrm{~d} q \frac{\left|f_{0}(q)\right|^{2}}{q} .
$$

Once again, we have equality iff. $F$ and $G$ are scalar multiples, which implies $f_{0}(q) \propto q$. Adding the other azimuthal terms to the right hand side will make it equal to the integral over all space, and equality will be reached iff. $f_{0}(q) \propto q$ and all other $f_{m}$ are zero,

$$
\left|\int_{0}^{k} \mathrm{~d} q f_{0}(q)\right|^{2} \leq \frac{k^{2}}{2} \sum_{m=-\infty}^{\infty} \int_{0}^{k} \mathrm{~d} q \frac{\left|f_{m}(q)\right|^{2}}{q}
$$




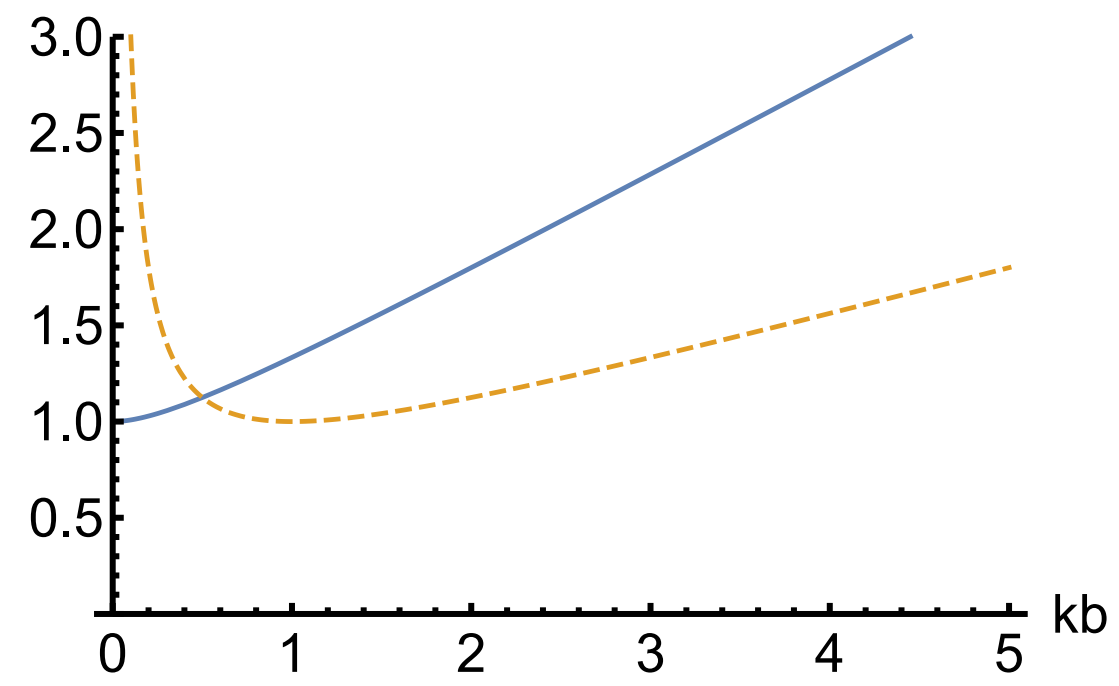

Figure 2.3: Focal measures $k \chi / 2 \pi$ (blue, solid) and $k^{2} \eta / 4 \pi$ (orange, dashed) for a $q^{k b}$ weight function. The $k b=0$ beam diverges, but represents the limit of tightest axial focus. On the other hand, radial focus is tightest at $k b=1$.

We therefore have

$$
\eta=\frac{2 \pi \sum_{m=-\infty}^{\infty} \int_{0}^{k} q^{-1}\left|f_{m}(q)\right|^{2}}{\left|\int_{0}^{k} f_{0}(k)\right|^{2}} \leq \frac{4 \pi}{k^{2}}=\frac{\lambda^{2}}{\pi} .
$$

For a $q^{k b}$ weight function, the measure is given by

$$
\eta=\pi \frac{(k b+1)^{2}}{k b} k^{-2}
$$

Contrast this with $\chi=\frac{2 \pi}{k} \frac{(k b+1)^{2}}{2 k b+1}$. Figure 2.3 gives plots of the ratio of the two measures to their minimum value. While $\chi$ is minimised in the limit $b \rightarrow 0, \eta$ has a minimum at $b=1$, and diverges as $b \rightarrow 0$. At $b=1 \eta$ achieves the minimum possible value, $\frac{4 \pi}{k^{2}}$. Hence, the proto-beam is in fact the unique tightest radially focused beam by this measure.

For a Gaussian beam, we have

$$
\eta=2 \pi \int_{0}^{\infty} \rho \mathrm{d} \rho e^{-\frac{k \rho^{2}}{b}}=\frac{\pi b}{k} .
$$


This is equal to $\pi / 2$ times the beam waist squared, $\eta=\frac{\pi}{2} w+0^{2}$. Thus for Gaussians, the $\chi$ and $\eta$ parameters have a direct correspondence to the commonly used parameters for measuring focal extent, $z_{R}$ and $w_{0}$.

On the other hand, for Gaussians these parameters are usually defined in terms of the decay properties of the beam. Gaussian beams have no zeros, and decay uniformly with increasing $\rho$ (if $\rho_{1}<\rho_{2}$ then $\left|\psi\left(\rho_{1}\right)\right|>\left|\psi\left(\rho_{2}\right)\right|$ ) Hence, the definition of the focal parameters is usually decided by distances at which the beam decays to $e^{-2}$ of the origin. However, for tightly focused beams, there will be zeros present, and non-uniform decay. In addition, the decay rates can be far slower, for example power law decay, compared to the greater than exponential decay of the Guassian beam.

\subsection{Optical focusing}

For particle beams the probability density is the natural quantity to use to measure the localisation. For classical light beams, it is the energy density. In free space, each component of $\boldsymbol{E}$ and $\boldsymbol{B}$ satisfies the wave equation, and hence the Helmholtz equation if the beam is monochromatic. The fields are represented by complex functions, with the real and imaginary parts giving two independent physical solutions. If we assume $e^{-i \omega t}$ time dependence, the spatial dependence will be given by complex solutions to the Helmholtz equation, i.e.

$$
\boldsymbol{E}=\left(\boldsymbol{E}_{r}+i \boldsymbol{E}_{i}\right) e^{-i \omega t} .
$$

Taking real parts, the physical field is given by

$$
\boldsymbol{E}=E_{r} \cos \omega t+E_{i} \sin \omega t .
$$

However, the cycle average energy density

$$
u_{A}=\frac{\omega}{2 \pi} \int_{t_{0}}^{t_{0}+\frac{2 \pi}{\omega}} \mathrm{d} t u(t)
$$

will be independent of $t_{0}$, and given by

$$
u_{A}=\frac{1}{8 \pi}\left(|E|^{2}+\left|B^{2}\right|\right) .
$$


Thus, if we use the cycle average energy density as our measure of the tightness of focus, we can construct the generalisation of equation 2.4.15). The condition for beam solutions is that the energy density per unit length is finite. This lets us define the axial focal measure as

$$
\chi=\frac{\int_{-\infty}^{\infty} \mathrm{d} z u_{A}(0, z)}{u_{A}\left(0, z_{0}\right)} .
$$

However, there is an issue in that electromagnetic beams cannot be azimuthally symmetric in all field components. Assume a beam, propagating in $z$ has an azimuthally symmetric electric field, $\frac{\partial \boldsymbol{E}}{\partial \phi}=0$. The $z$ component of the curl of $\boldsymbol{E}$ gives

$$
\frac{\partial E_{y}}{\partial x}-\frac{\partial E_{x}}{\partial y}=0
$$

This implies

$$
\cos \phi \frac{\partial E_{y}}{\partial \rho}=\sin \phi \frac{\partial E_{x}}{\partial \rho} .
$$

Since we have assumed the field is azimuthally symmetric, the $\rho$ derivative must be as well. However, evaluating at $\phi=0, \pi / 2$ gives us $\frac{\partial E_{y}}{\partial \rho}=\frac{\partial E_{x}}{\partial \rho}=0$. This cannot represent a beam, as a beam must be radially finite, and thus cannot have components independent of $\rho$. The same proof can be applied to the magnetic field. However, the cycle average energy can be azimuthally symmetric. A sufficient criteria for this is having the azimuthal dependence of the complex fields given by $e^{i m \phi}$. For example, the TE Hertz potential

$$
\Pi=\frac{E_{0}}{k^{2}} e^{i m \phi} \int_{0}^{k} \mathrm{~d} q f(k, q) e^{i q z} J_{m}(\kappa \rho) \hat{z},
$$

will give an azimuthally symmetric cycle average energy. In general such a beam will be hollow: the energy density being zero on the beam axis, due to $J_{m}(0)=0$ for $m \neq 0$. However, we have

$$
\frac{\partial J_{m}(\alpha)}{\partial \alpha}=\frac{1}{2}\left(J_{m-1}(\alpha)-J_{m+1}(\alpha)\right) .
$$

Since the electric field is proportional to the curl of $\Pi$, and the magnetic field the double curl, the first and second derivatives of $J_{m}(\kappa \rho)$ with respect to $\rho$ will 
enter the expression for the energy density. The first derivative of $J_{1}(k \rho)$ will give rise to terms proportional to $J_{0}(k \rho)$, and the second derivative gives rise to terms proportional to $J_{1}(k \rho) / k \rho$, both of which are non-zero at the origin. Similarly, the second derivative of $J_{2}(k \rho)$ includes a term proportional to $J_{0}(k \rho)$. As a result, the $m=1$ beam will be non-hollow in both the $\boldsymbol{E}$ and $\boldsymbol{B}$ contributions to $u$, and the $m=2$ beam will be non-hollow due to $\boldsymbol{B}$.

\subsection{Transverse modes}

All solutions of Maxwell's equations have $\boldsymbol{E}$ and $\boldsymbol{B}$ transverse to the local propagation direction: this is a simple consequence of the fact that the local propagation is given by the Poynting vector $\boldsymbol{S}=\frac{1}{4 \pi} \boldsymbol{E} \times \boldsymbol{B}$, and all vector fields are transverse to their cross product. However, the TE, TM and TEM modes discussed in literature do not refer to the local propagation, rather they refer to the global propagation, given by the beam axis direction. Lekner shows [20] that there can be no TEM beam, in the sense of having both the electric and magnetic fields that are everywhere transverse to the beam axis. As the Poynting vector is transverse to both $\boldsymbol{E}$ and $\boldsymbol{B}$, this also implies the Poynting vector cannot point along the beam axis everywhere. Indeed, if the Poynting flux was everywhere in the same direction, such a beam would have an energy density unchanged by propagation direction in this direction. As a result, this would constitute a nondiffracting beam, which is not physical, as non-diffracting beams carry infinite energy content [27].

Lekner [8, sec. 20.1] shows that the time averaged Poynting flux through a plane radial to the propagation direction is a beam invariant

$$
\frac{\partial}{\partial z_{0}} \int_{z=z_{0}} \mathrm{~d}^{2} r S_{z}=0
$$

Thus, although locally the Poynting flux will not point along the beam direction everywhere, the radial integral will.

Although there are no finite TEM beams, it is possible to have either the electric, or magnetic field exclusively transverse to the beam axis, the TE and 
TM modes. I shall now show the TE beam will always be given by a Hertz potential purely along the beam axis. Consider an arbitrary transverse Hertz potential

$$
\boldsymbol{\Pi}=\frac{A_{0}}{k} \sum_{m=\infty}^{\infty} \int_{0}^{k} \frac{\mathrm{d} q}{k^{2}} e^{i(q z+m \phi)} J_{m}(\kappa \rho)\left(f_{m}(k, q) \hat{\rho}+g_{m}(k, q) \hat{\phi}\right) .
$$

The curl gives the vector potential, proportional to the electric field. Note we are assuming the scalar potential is zero, this is because for a TE beam we would require a scalar potential that is independent of $z$, which will not represent a beam. Thus, the $\hat{z}$ component of $\boldsymbol{A}$ is given by

$$
\begin{aligned}
A_{z} & =A_{0} \sum_{m=-\infty}^{\infty} \int_{0}^{k} \mathrm{~d} q e^{i(q z+m \phi)} \\
& \times\left(\frac{\kappa}{k} J_{m-1}(\kappa \rho) g_{m}(k, q)+\frac{1}{k \rho} J_{m}(\kappa \rho)\left(g_{m}(k, q)-m\left(f_{m}(k, q)+g_{m}(k, q)\right)\right)\right) \\
= & 0 .
\end{aligned}
$$

Integrating $A_{z} e^{-i m^{\prime} \phi}$ over $\phi$ will isolate a single Fourier mode $A_{m^{\prime}}$, and further integrating $A_{m^{\prime}} e^{-i q^{\prime} z}$ over $z$ will isolate a single value of $q, \kappa$. Thus for all $m, q$ we must have

$$
\kappa \rho J_{m-1}(\kappa \rho)+J_{m}(\kappa \rho)\left(g_{m}(k, q)-m\left(f_{m}(k, q)+g_{m}(k, q)\right)\right)=0 .
$$

This must be true for all $\rho$. Evaluating at a zero of $J_{m}(\kappa \rho)$ gives $g_{m}(k, q)=0$, as Bessel functions of different orders do not share zeros. Evaluating at a zero of $J_{m-1}(\kappa \rho)$ gives $f_{m}(k, q)=0$. We can see that for a TE beam the scalar potential, and transverse components of the Hertz potential must be zero. Thus, every TE beam can be written using a Hertz potential

$$
\Pi=\frac{E_{0}}{k^{2}} \psi(\rho, \phi, z) \hat{z}
$$

It should also be noted that although the fields are always perpendicular to the local propagation direction, they need not be transverse to each other everywhere. First, while it is true $\boldsymbol{E} \cdot \boldsymbol{B}$ is a Lorentz scalar field, this alone does not imply it is zero everywhere. Neither does the fact that for monochromatic beams 
$\boldsymbol{B}$ is proportional to the curl of $\boldsymbol{E}$; although for a cross product the vectors are orthogonal to their product, a vector is not always orthogonal to its curl. In general, in areas where the beam can be approximated as a plane wave, $\boldsymbol{E} \cdot \boldsymbol{B}=0$, while in areas of large wave-front curvature this may not be the case.

Now that we have established the properties of finite optical beams, we return to the question of their focusing parameter. Rather than a Bessel beam expansion, it will be useful to give a wave-function in terms of a Fourier transform over the sphere of radius $k$ in Fourier space,

$$
\psi(\boldsymbol{r})=\int_{0}^{\pi / 2} \int_{-\pi}^{\pi} \sin \alpha \mathrm{d} \alpha \mathrm{d} \beta f(\alpha, \beta) e^{i \boldsymbol{k}(\alpha, \beta) \cdot \boldsymbol{r}},
$$

where we have restricted $\alpha<\pi / 2$ to represent a forward propagating beam along $z$. We also note that

$$
\boldsymbol{k}=k(\sin \alpha \cos \beta \hat{x}+\sin \alpha \sin \beta \hat{y}+\cos \alpha \hat{z})
$$

We shall now derive a useful orthogonality relation

$$
\int_{\mathbb{R}^{2}} \mathrm{~d}^{2} r e^{i \boldsymbol{r} \cdot\left(\boldsymbol{k}(\alpha, \beta)-\boldsymbol{k}\left(\alpha^{\prime}, \beta^{\prime}\right)\right)}=\frac{(2 \pi)^{2} \delta\left(\alpha-\alpha^{\prime}\right) \delta\left(\beta-\beta^{\prime}\right)}{k^{2} \sin \alpha \cos \alpha}, \quad \alpha \in[0, \pi / 2],
$$

where $\mathrm{d}^{2} r=\mathrm{d} x \mathrm{~d} y$. First, the generating function for Bessels is

$$
e^{\frac{1}{2} \zeta\left(\tau-\tau^{-1}\right)}=\sum_{m=-\infty}^{\infty} \tau^{m} J_{m}(\zeta)
$$

Next, a plane wave in cylindrical coordinates can be written as

$$
\begin{aligned}
e^{i \boldsymbol{k}(\boldsymbol{\alpha}, \boldsymbol{\beta}) \cdot \boldsymbol{r}}=\exp (i k(z \cos \alpha+\rho \sin \alpha(\cos \phi \cos \beta+\sin \phi \sin \beta))) & \\
& =e^{i k(z \cos \alpha+\rho \sin \alpha \cos (\phi-\beta))} .
\end{aligned}
$$

If we substitute $\zeta \rightarrow k \rho \sin \alpha, \tau \rightarrow i e^{i(\phi-\beta)}$, equation (2.7.9) gives the plane wave expansion in cylindrical coordinates,

$$
e^{i \boldsymbol{k} \cdot \boldsymbol{r}}=e^{i k z \cos \alpha} \sum_{m=-\infty}^{\infty} i^{m} e^{i m(\phi-\beta)} J_{m}(k \rho \sin \alpha) .
$$


The relevant orthogonality integrals are

$$
\int_{0}^{2 \pi} e^{i\left(m-m^{\prime}\right) \phi}=2 \pi \delta_{m, m^{\prime}}, \quad \int_{0}^{\infty} \rho \mathrm{d} \rho J_{m}(\kappa \rho) J_{m}\left(\kappa^{\prime} \rho\right)=\frac{\delta\left(\kappa-\kappa^{\prime}\right)}{\kappa^{\prime}} .
$$

For $\kappa=k \sin \alpha$, we can use the composition property of the Dirac delta,

$$
\delta(f(x))=\sum_{n} \frac{\delta\left(x-x_{n}\right)}{\left|f^{\prime}\left(x_{n}\right)\right|}
$$

where the sum is over the $n$ zeros of $f(x)$. As we have chosen to restrict $\alpha, \alpha^{\prime}$ to the interval $[0, \pi / 2]$, viewing the argument of the delta as a function of $\alpha$, there is only one point in this interval where $\sin \alpha=\sin \alpha^{\prime}$; when $\alpha=\alpha^{\prime}$. This gives

$$
\frac{\delta\left(k \sin \alpha-k \sin \alpha^{\prime}\right)}{k \sin \alpha^{\prime}}=\frac{\delta\left(\alpha-\alpha^{\prime}\right)}{k^{2} \sin \alpha^{\prime} \cos \alpha^{\prime}} .
$$

A useful identity is that the the Dirac delta over the interval $[-\pi, \pi]$ can be represented by (NIST Handbook, section 10.12, |6])

$$
2 \pi \delta\left(\beta-\beta^{\prime}\right)=\sum_{m=-\infty}^{\infty} e^{i m\left(\beta-\beta^{\prime}\right)} .
$$

The integral of the two plane waves is given

$$
\begin{array}{r}
\int_{\mathbb{R}^{2}} \mathrm{~d}^{2} r e^{i \boldsymbol{r} \cdot\left(\boldsymbol{k}(\alpha, \beta)-\boldsymbol{k}\left(\alpha^{\prime}, \beta^{\prime}\right)\right)}=e^{i k z\left(\cos \alpha-\cos \alpha^{\prime}\right)} \\
\times \sum_{m, m^{\prime}=-\infty}^{\infty} \int_{-\pi}^{\pi} \mathrm{d} \phi \int_{0}^{\infty} \rho \mathrm{d} \rho i^{m-m^{\prime}} e^{i\left(m-m^{\prime}\right) \phi} e^{-i\left(m \beta-m^{\prime} \beta^{\prime}\right)} \\
\times J_{m}(k \rho \sin \alpha) J_{m^{\prime}}\left(k \rho \sin \alpha^{\prime}\right) .
\end{array}
$$

Applying the orthogonality relations make the integral equal to

$$
\sum_{m=-\infty}^{\infty} 2 \pi e^{i m\left(\beta-\beta^{\prime}\right)} \frac{\delta\left(\alpha-\alpha^{\prime}\right)}{k^{2} \sin \alpha \cos \alpha}=\frac{(2 \pi)^{2} \delta\left(\alpha-\alpha^{\prime}\right) \delta\left(\beta-\beta^{\prime}\right)}{k^{2} \sin \alpha \cos \alpha} .
$$

This proves equation (2.7.8).

An advantage of the TE beam is that it has an incredibly simple expression for the cycle average energy over the radial plane. Take the Hertz potential to be

$$
\boldsymbol{\Pi}=-i \frac{A_{0}}{k} \hat{z} \int_{-\pi}^{\pi} \mathrm{d} \beta \int_{0}^{\pi / 2} \mathrm{~d} \alpha e^{i \boldsymbol{k} \cdot \boldsymbol{r}}
$$


The vector potential is given by

$$
\boldsymbol{A}=A_{0} \int_{-\pi}^{\pi} \mathrm{d} \beta \int_{0}^{\pi / 2} \mathrm{~d} \alpha f(\alpha, \beta) e^{i \boldsymbol{k} \cdot \boldsymbol{r}} \hat{k} \times \hat{z}
$$

Note that $\hat{k} \times \hat{z}=\sin \alpha \sin \beta \hat{x}-\sin \alpha \cos \beta \hat{y}$. The squared magnitude of this vector is $\sin ^{2} \alpha$. The electric field is given by $\boldsymbol{E}=i k \boldsymbol{A}$. The integral of $|E|^{2}$ radially gives

$$
\int_{\mathbb{R}^{2}} \mathrm{~d}^{2} r|E|^{2}=(2 \pi)^{2} A_{0}^{2} \int_{-\pi}^{\pi} \mathrm{d} \beta \int_{0}^{\pi / 2} \mathrm{~d} \alpha \frac{\sin \alpha}{\cos \alpha}|f(\alpha, \beta)|^{2},
$$

having applied the orthogonality relation (2.7.8). $\boldsymbol{B}$ is given by $\nabla \times \boldsymbol{A}$, and we have

$$
\hat{k} \times(\hat{k} \times \hat{z})=\sin \alpha(\cos \alpha \cos \beta \hat{x}+\cos \alpha \sin \beta \hat{y}-\sin \alpha \hat{z}) .
$$

The squared magnitude of this vector is also $\sin ^{2} \alpha$. The integral of $|B|^{2}$ radially is the same as that of $|E|^{2}$. This is expected as the electric and magnetic fields contain the same energy content $[28 \mid \mathrm{s}$. This gives the cycle average energy per unit length to be

$$
u=\pi A_{0}^{2} \int_{-\pi}^{\pi} \mathrm{d} \beta \int_{0}^{\pi / 2} \frac{\sin \alpha}{\cos \alpha}|f(\alpha, \beta)|^{2} .
$$

The relevant Cauchy-Schwartz inequality for vector fields and an integral measure $\mathrm{d} \mu$ is

$$
\left|\int \boldsymbol{F} \cdot \boldsymbol{G}^{*} \mathrm{~d} \mu\right|^{2} \leq \int|F|^{2} \mathrm{~d} \mu \times \int|G|^{2} \mathrm{~d} \mu .
$$

Our spherical measure is $\mathrm{d} \mu=\sin \alpha \mathrm{d} \alpha \mathrm{d} \beta$. We can set $\boldsymbol{F}=(\cos \alpha)^{-1 / 2} f(\alpha, \beta) \hat{k} \times$ $\hat{z}$, for $\boldsymbol{E}$, and similarly for $\boldsymbol{B}$, to find an inequality involving the value at the origin. The relevant choice for $\boldsymbol{G}$ is $\boldsymbol{G}=(\cos \alpha)^{1 / 2} \hat{e}$, where $\hat{e}$ is the unit vector in the direction of the field at the origin. Note that $\hat{e}$ is a constant vector, while $\hat{k} \times \hat{z}$ depends on $\alpha, \beta$.

$$
\left|\int_{-\pi}^{\pi} \mathrm{d} \beta \int_{0}^{\pi / 2} \sin \alpha \mathrm{d} \alpha f(\alpha, \beta)(\hat{k} \times \hat{z}) \cdot \hat{e}\right|^{2} \leq \pi \int_{-\pi}^{\pi} \mathrm{d} \beta \int_{0}^{\pi / 2} \mathrm{~d} \alpha \frac{\sin \alpha}{\cos \alpha}|f(\alpha, \beta)|^{2} .
$$

We can see that the left-hand side is $\left|E_{0}\right|^{2} / k^{2} A_{0}^{2}$, where $\boldsymbol{E}_{0}$ is the field at the maximum of energy, which we have assumed without loss of generality to be at 
the origin. The right-hand side is $u / A_{0}^{2}$. We will obtain the same inequality for $\left|B_{0}\right|^{2}$. We therefore have

$$
\left|E_{0}\right|^{2}+\left|B_{0}\right|^{2} \leq 2 k^{2} u, \quad 8 \pi u_{0} \leq 2 k^{2} u
$$

This gives

$$
\eta_{T E}=\frac{u}{u_{0}} \geq \frac{4 \pi}{k^{2}}=\frac{\lambda^{2}}{\pi} .
$$

Unlike the scalar case, equality cannot be reached for an electromagnetic beam. For the vector field Cauchy-Schwartz inequality, equality is reached iff. $\boldsymbol{F}$ and $\boldsymbol{G}$ are linearly proportional by the same amount everywhere. As our choice of $\boldsymbol{G}$ is the same direction everywhere, this would require the electric and magnetic fields be of uniform linear polarisation, which as discussed previously was disproved by Lekner. We shall examine some specific examples, and show that for some families of weight functions the lower bound is approximately 2.7 times higher.

As we have discussed previously, to have a non-hollow beam the weight function needs to have an $e^{ \pm i \beta}$ dependence. The equivalent of the $q^{k b}$ scalar beam for an electromagnetic beam is given by

$$
f(\alpha, \beta)=\cos ^{k b} \alpha \sin \beta .
$$

The fields at the origin are given by

$$
\begin{aligned}
& \boldsymbol{E}_{0}=k A_{0} \int_{-\pi}^{\pi} \mathrm{d} \beta \int_{0}^{\pi / 2} \mathrm{~d} \alpha \sin \alpha \cos ^{k b} \alpha \hat{e}_{1}(\alpha, \beta)=\frac{-\pi k A_{0}}{1+k b}, \\
& \boldsymbol{B}_{0}=k A_{0} \int_{-\pi}^{\pi} \mathrm{d} \beta \int_{0}^{\pi / 2} \mathrm{~d} \alpha \sin \alpha \cos ^{k b} \alpha \hat{e}_{2}(\alpha, \beta)=\frac{\pi k A_{0}}{2+k b} .
\end{aligned}
$$

The time average energy density at the origin is given by

$$
u_{0}=\frac{\pi k^{2} A_{0}^{2}\left(2 k^{2} b^{2}+6 k b+5\right)}{8(k b+1)^{2}(k b+2)^{2}} .
$$

The energy density per unit length of the beam is

$$
u=\pi A_{0}^{2} \int_{-\pi}^{\pi} \mathrm{d} \beta \int_{0}^{\pi / 2} \mathrm{~d} \alpha \sin \alpha \cos ^{2 k b-1} \alpha \sin ^{2} \beta=\frac{\pi^{2} A_{0}^{2}}{2 k b} .
$$




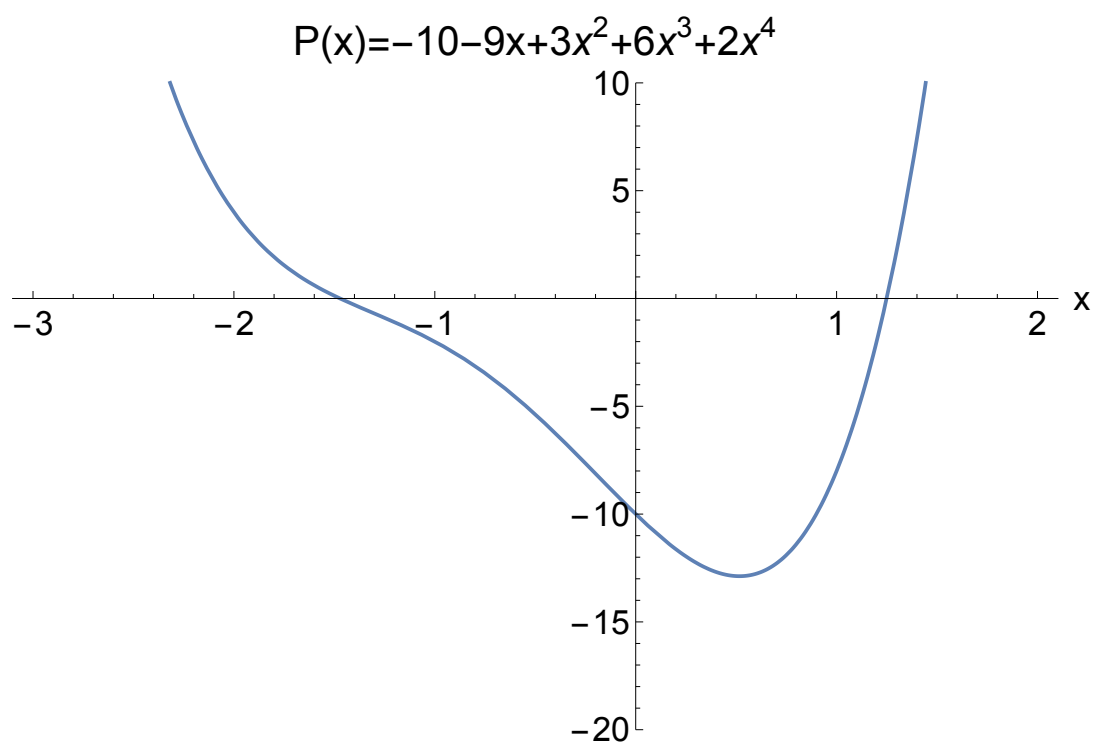

Figure 2.4: Quartic factor in 2.7.32), the positive root of which gives the value of $k b$ that results in the beam of tightest focus, for a weight function of $\cos ^{k b} \alpha \sin \beta$.

The ratio is therefore

$$
\eta=\frac{u}{u_{0}}=\frac{4 \pi}{k^{2}} \frac{(k b+1)^{2}(k b+2)^{2}}{k b(2 k b(k b+3)+5)} .
$$

We can find the $k b$ that minimises $\eta$. The numerator of the $k b$ derivative of $\eta$ is given by

$$
(k b+1)(k b+2)\left(2 k^{4} b^{4}+6 k^{3} b^{3}+3 k^{2} b^{2}-9 k b-10\right) .
$$

The energy density is divergent for $k b \leq-1$, so we are interested in the roots of the quartic. The real, positive root of this can be obtained either through the quartic formula, or numerically, and comes out to $k b \approx 1.24907$. At this value, $\eta$ reaches its minimum of $\eta \approx 2.7378 \times 4 \pi / k^{2}$. It would seem that the vectorial nature of an electromagnetic beam results in a loosening of focus compared to a scalar beam. As our lowest bound so far is lower than this, we cannot definitely say if this is the tightest a beam can be focused. In addition to arbi- 
trary weight functions, we can also consider arbitrary combinations of $\hat{e}_{1}$ and $\hat{e}_{2}$, which complicates the analysis.

\subsection{Discussion}

In this chapter, we have examined the invariants and focal limits of exact solutions to the Helmholtz equation. We have shown that probability density per unit length can be an invariant as long as the beam consists of only forward propagating components. For beams with backward propagating components, the probability density oscillates, but the average probability density will be an invariant, and equal to the sum of the forward and backward invariants.

We examined how tightly a beam can be focused. The usual measures, such as the radius at which the beam drops to $e^{-2}$ in intensity, are shown to be inadequate for tightly focused beams, which are oscillatory in their intensity profiles. Dorn et al [29] demonstrate experimentally the tightest possible focus based on FWHM spot size parameter. We have seen however, that this is not necessarily the tightest possible focus, based on the concentration of the beam intensity to the maximum. We introduced polarisation measures $\chi$ and $\eta$, which relate the axial and radial integrals of intensity respectively, to the peak intensity. The lower bounds

$$
\chi \geq \frac{2 \pi}{k}, \quad \eta \geq \frac{4 \pi}{k^{2}},
$$

were shown to be achieved (asymptotically in the case of $\chi$ ) for azimuthally symmetric beams with weight functions given by a constant, and $f_{0}(q) \propto q$ respectively. This shows the proto-beam is in fact the unique limit of how tightly focused a scalar beam could be. It is our view that if a focal volume is desired, the combination $\eta \times \chi$ is a useful quantity, however we have yet to derive a rigorous lower bound for this product.

We also derived a similar measure for electromagnetic beams. However, this is where the limitation of our measure becomes apparent: our measure uses the peak value, however many beams can be hollow, including tightly focused electromagnetic beams. For a hollow beam, or one which is not maximum at the 
focus, a different measure is required. For paraxial beams, a common parameter is to compare the integrals of different moments of the beam. Specifically, define the nth radial moment of a beam to be

$$
\int_{\mathbb{R}^{2}} \mathrm{~d}^{2} r \rho^{n}|\psi(\rho, \phi, z)|^{2}=\int_{-\pi}^{\pi} \mathrm{d} \phi \int_{0}^{\infty} \mathrm{d} \rho \rho^{n+1}|\psi(\rho, \phi, z)|^{2} .
$$

Ratios of different moments give different estimates of spot size, for example one can take the ratio of the first moment to the zeroth (the integral of intensity over all space). In this terminology, our $\eta$ parameter is the ratio of the zeroth moment to the peak.

For paraxial beams, the falloff of the intensity is Gaussian type, $e^{-\frac{k \rho^{2}}{b}}$. For exact solutions of the Helmholtz equation, it can be power law. As mentioned in the introduction, the proto-beam has a Jinc profile radially, which has an asymptotic expansion of

$$
\frac{J_{1}(k \rho)}{k \rho} \sim-\sqrt{\frac{2}{\pi}} \cos \left(k \rho+\frac{\pi}{4}\right) k^{-3 / 2} \rho^{-3 / 2}+\mathscr{O}\left(k^{-5 / 2} \rho^{-5 / 2}\right) .
$$

As such, the falloff in intensity is $\rho^{-3}$. For the integrals to converge the integrand must have a falloff higher than $\rho^{-1}$. For the first moment integral, the integrand falls off with exactly $\rho^{-1}$ which is divergent. This is an example of a physical beam with finite invariants, that has a divergent moment integral.

Moment integrals can be obtained via the Laplace transform of $r|\psi|^{2}$. Consider

$$
\Psi(s, z)=\int_{-\pi}^{\pi} \mathrm{d} \phi \int_{0}^{\infty} \mathrm{d} \rho \rho|\psi(\rho, \phi, z)|^{2} e^{-s \rho} .
$$

The derivative property of the Laplace transform is that if $F(s)$ is the Laplace transform of $f(r)$,

$$
\frac{\mathrm{d}^{n} F}{\mathrm{~d} s^{n}}=(-)^{n} \int_{0}^{\infty} \mathrm{d} r r^{n} f(r) .
$$

Evaluating the nth derivative of the Laplace transform at $s=0$ gives the nth moment. Evaluating the Laplace transform at $s=0$ gives the integral of intensity over all space. The moment will be undefined if the Laplace transform does not have an nth derivative at the origin. 
A possible solution to the divergence of the moment integrals is to exponentially regulate the integrals. This is equivalent to evaluating the Laplace transform and its derivatives at some non-zero value of $s$. However, we can use the Gaussian beam as an example to show how this may not be desirable.

For the Gaussian beam at $z=0$, the Laplace transform is

$$
\Psi_{G}(s, 0)=\int_{0}^{\infty} \rho \mathrm{d} \rho e^{-\frac{k \rho^{2}}{b}}=\frac{b\left(2 k-\sqrt{\pi} s \sqrt{b k} e^{\frac{b s^{2}}{4 k}} \operatorname{erfc}\left(\frac{1}{2} s \sqrt{\frac{b}{k}}\right)\right)}{4 k^{2}} .
$$

The negated first derivative is

$$
\frac{b\left(\sqrt{\pi} \sqrt{b k} e^{\frac{b s^{2}}{4 k}}\left(b s^{2}+2 k\right) \operatorname{erfc}\left(\frac{1}{2} s \sqrt{\frac{b}{k}}\right)-2 b k s\right)}{8 k^{3}} .
$$

Evaluating at $s=0$ and taking the ratio gives the first moment measure

$$
\sigma(0,0)=-\left.\frac{1}{\Psi(0)} \frac{\mathrm{d} \Psi}{\mathrm{d} s}\right|_{s=0}=\sqrt{\frac{\pi b}{4 k}} .
$$

This is clearly proportional to the beam waist, and grows without bound as $b$ increases. However, consider evaluating at $s=k$,

$$
\sigma(k, 0)=\frac{b\left(\sqrt{\pi} e^{\frac{b k}{4}}(b k+2) \operatorname{erfc}\left(\frac{\sqrt{b k}}{2}\right)-2 \sqrt{b k}\right)}{4 \sqrt{b k}-2 \sqrt{\pi} b k e^{\frac{b k}{4}} \operatorname{erfc}\left(\frac{\sqrt{b k}}{2}\right)} .
$$

As $b$ tends to infinity, this tends to a limiting value of $2 / k$. As such, for weakly focused beams this parameter does not help differentiate their spot size.

An alternative is to regulate only the first moment, and leave the total unregulated. This is also undesirable: for the Gaussian beam this gives

$$
\bar{\sigma}(s, 0)=\frac{\sqrt{b}\left(\sqrt{\pi} e^{\frac{b s^{2}}{4 k}}\left(b s^{2}+2 k\right) \operatorname{erfc}\left(\frac{1}{2} s \sqrt{\frac{b}{k}}\right)-2 s \sqrt{b k}\right)}{4 k^{3 / 2}} .
$$

A plot of this parameter for $s=k$ shows an initial increase, which reaches a turning point, and decreases of increasing $k b$ (Figure 2.5). This is highly undesirable behaviour, as the focal parameter should unambiguously distinguish between beams of different focal properties. 


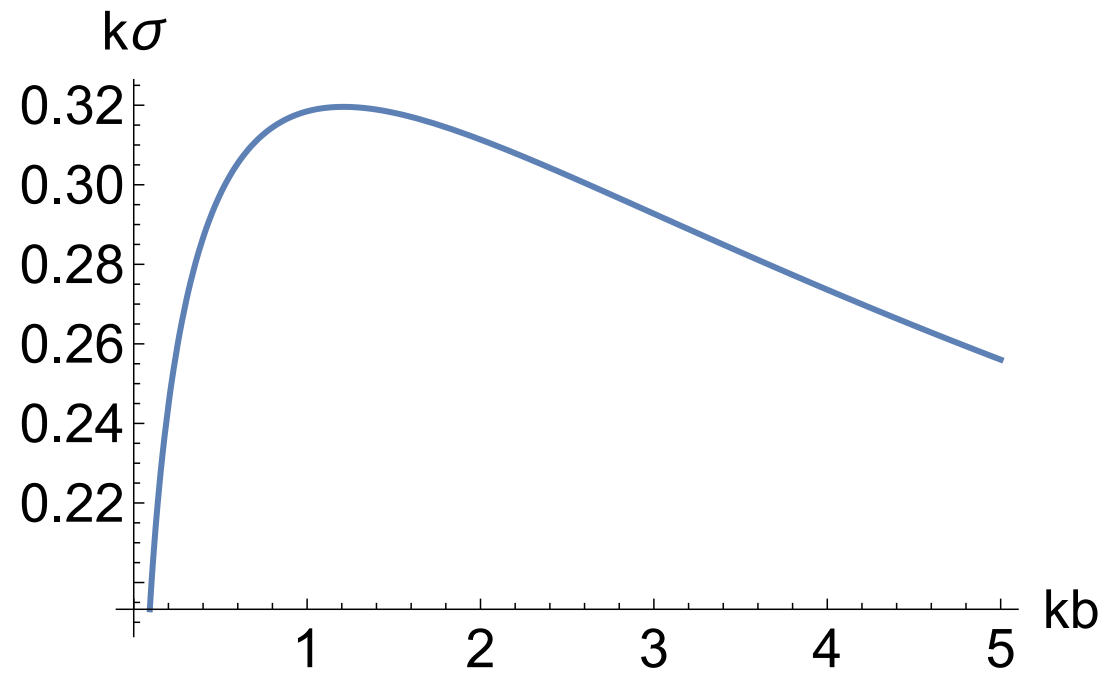

Figure 2.5: Plot of equation 2.8.10. The focal parameter $k \sigma$ grows with increasing $k b$, up to a turning point. Beyond this it decreases. This is undesirable, as the focal parameter should increase with a loosening of focus (increasing $k b$ ).

A final attempt at defining a focal parameter can be to compare a regulated zeroth moment to the unregulated zeroth moment. As the zeroth moment must be convergent for physical beams, this parameter is guaranteed to converge. The regulated zeroth moment emphasises the components closer to the focus than the unregulated integral. Hence, our candidate for the focal parameter is

$$
\sigma(s, z)=\frac{\Psi(0, z)}{\Psi(s, z)} .
$$

In effect, this takes the opposite approach of the second moment parameter; the second moment enhances the weighting of intensity further from the focus in the numerator. Our approach instead diminishes the weighting of intensity closer to the focus in the denominator.

Different beams must be compared at the same value of $s$ for this to be useful, $s=k$ is a natural choice. For the Gaussian beam we get

$$
\sigma_{G}(s, 0)=\frac{2 k}{2 k-\sqrt{\pi} s \sqrt{b k} e^{\frac{b s^{2}}{4 k}} \operatorname{erfc}\left(\frac{1}{2} s \sqrt{\frac{b}{k}}\right)} .
$$


This parameter grows monotonically with $k b$ for fixed $s$, and is a good candidate for a focal parameter. Asymptotically, for large $k b$ and $s=k$, we have

$$
\sigma_{G}(k, 0) \sim \frac{k b}{2}-\frac{12}{k b}+3 .
$$

The issue with this parameter is that it relies on the Laplace transform of the modulus. For general beams this will involve integrals of the form

$$
\int_{0}^{\infty} \mathrm{d} \rho \iint_{0}^{k} \mathrm{~d} q \mathrm{~d} q^{\prime} \rho J_{m}(\kappa \rho) J_{m}\left(\kappa^{\prime} \rho\right) e^{-s \rho} f(k, \kappa) f\left(k, \kappa^{\prime}\right) .
$$

For $s=0$ the Bessel integral gives an orthogonality relation, however, for arbitrary $s$ the integral over $\rho$ will be given in terms of elliptic integrals $|30|$. As a result, evaluating this focal parameter symbolically may not be tractable for arbitrary beams.

We may also consider alternative choices of regulator. A natural choice is a Gaussian regulator, letting us define a 'Gaussian transform' of a function $\psi(\rho, \phi, z)$ to be

$$
\Psi(s, z)=\int_{-\pi}^{\pi} \mathrm{d} \phi \int_{0}^{\infty} \mathrm{d} \rho \rho e^{-s^{2} \rho^{2}}|\psi(\rho, \phi)|^{2}
$$

We can then proceed as we did with the Laplace transforms, to define the parameter

$$
\sigma(s, z)=\frac{\Psi(0, z)}{\Psi(s, z)}
$$

For the Gaussian beam, this evaluates to

$$
\sigma_{G}(s, z)=\frac{s^{2}\left(b^{2}+z^{2}\right)}{k b}+1 .
$$

This is far simpler than the case using Laplace transforms. Compare this with the $e^{-2}$ spot size of $k^{2} \rho^{2}=2 k^{2}\left(b^{2}+z^{2}\right) / k b$. We can see that the growth with $b, z$ is identical for $s=\sqrt{2 k}$.

On the other hand, the situation for general beams is only moderately improved. The integral of interest becomes

$$
\int_{0}^{\infty} \mathrm{d} \rho \iint_{0}^{k} \mathrm{~d} q \mathrm{~d} q^{\prime} \rho J_{m}(\kappa \rho) J_{m}\left(\kappa^{\prime} \rho\right) e^{-s^{2} \rho^{2}} f(k, \kappa) f\left(k, \kappa^{\prime}\right) .
$$


Rather than elliptic integrals, the result is instead given in terms of a double integral over a product involving the Bessel I function [6, eq. 10.22.67],

$$
\int_{0}^{\infty} \rho e^{-s^{2} \rho^{2}} J_{0}(\kappa \rho) J_{0}\left(\kappa^{\prime} \rho\right)=\frac{1}{2 s^{2}} \exp \left(-\frac{\kappa^{2}+\kappa^{\prime 2}}{4 s^{2}}\right) I_{0}\left(\frac{\kappa \kappa^{\prime}}{2 s^{2}}\right)
$$




\section{Chapter 3}

\section{Nonexistence of exact solutions agreeing with the Gaussian beam on the beam axis or in the focal plane}

The following chapter includes verbatim a paper I have co-authored with John Lekner, produced as a part of this thesis project. It has been accepted for publication in Optics Communications [22]. In it, we examine attempts to find exact solutions of the Helmholtz equation that match the Gaussian beam in the focal plane. I use Hankel transforms to show this is not possible, as such beams will be evanescent and grow exponentially. In addition, I also show that BesselGauss beams cannot be matched in the focal plane for the same reason. We also show the more surprising result that the Gaussian beam cannot be matched on the beam axis. Lekner formulates the problem into an integral equation, which I show is inconsistent. This is surprising, as the paraxial approximation is supposed to be accurate close to the beam axis. Lekner shows that there does exist a family of exact solutions that approximates the Gaussian beam for large focal parameter $k b$, and uses series expansions to find its divergence angle. 


\section{Abstract}

Solutions of the Helmholtz equation which describe electromagnetic beams (and also acoustic or particle beams) are discussed. We show that an exact solution which reproduces the Gaussian beam waveform on the beam axis does not exist. This is surprising, since the Gaussian beam is a solution of the paraxial equation, and thus supposedly accurate on and near the beam axis. Likewise, a solution of the Helmholtz equation which exactly reproduces the Gaussian beam in the focal plane does not exist. We show that the last statement also holds for Bessel-Gauss beams. However, solutions of the Helmholtz equation (one of which is discussed in detail) can approximate the Gaussian waveform within the central focal region.

\subsection{Introduction}

Acoustic, electromagnetic and particle beams are described by solutions of the Helmholtz equation

$$
\left(\nabla^{2}+k^{2}\right) \psi=0
$$

Equation (3.1.1) results respectively from the linearized hydrodynamic equations, the Maxwell equations, and the Schrödinger equation. The wavenumber $k=\omega / c$ (with $\omega$ the angular frequency and $c$ the speed of sound or the speed of light), or is related to the energy per particle, which for the particle mass $M$ is $\hbar^{2} k^{2} / 2 M$.

The widely used but approximate solution known as the Gaussian beam ([31], Section 16.7, [8], Section 20.3) is

$$
\psi_{G}(x, y, z)=\frac{b}{b+i z} \exp \left(i k z-\frac{k\left(x^{2}+y^{2}\right)}{2(b+i z)}\right) .
$$

$\psi_{G}$ is the fundamental mode solution of the paraxial equation, obtained by setting $\psi=e^{i k z} G$ in the Helmholtz equation and then neglecting the term $\partial_{z}^{2} G$ in 
the resulting equation for $G$ (given below). This amounts to assuming that the dominant $z$-dependence of the beam lies in the $e^{i k z}$ factor (when propagation is in the $z$ direction, as is assumed here). For axially symmetric solutions we omit the azimuthal derivative, so the Helmholtz equation in cylindrical coordinates, with $\rho=\sqrt{x^{2}+y^{2}}$ the distance from the beam axis, takes the form

$$
\left(\partial_{\rho}^{2}+\rho^{-1} \partial_{\rho}+\partial_{z}^{2}+k^{2}\right) \psi=0 .
$$

The substitution $\psi=e^{i k z} G$ gives an equation for $G$, namely $\left(\partial_{\rho}^{2}+\rho^{-1} \partial_{\rho}+2 i k \partial_{z}+\right.$ $\left.\partial_{z}^{2}\right) G=0$, in paraxial form $\left(\partial_{\rho}^{2}+\rho^{-1} \partial_{\rho}+2 i k \partial_{z}\right) G \approx 0$. This paraxial equation has as fundamental solution $G=\frac{b}{b+i z} \exp \left(-\frac{k \rho^{2}}{2(b+i z)}\right)$, thus giving us $\psi_{G}$ of equation (3.1.2). Higher modes may be obtained by differentiation of $\psi_{G}$ with respect to $x, y$ or $z$, since the differential equations are unchanged by translation in any coordinate. $\psi_{G}$ depends on the wavenumber $k$ and on the length $b$, which gives the longitudinal extent of the focal region. The transverse extent in the focal plane is given by $w_{0}=\sqrt{2 b / k}$. Thus the Gaussian fundamental mode is characterized by a single dimensionless parameter $k b$. It may seem plausible that when $k b \gg 1$ (focal region large longitudinally compared to $k^{-1}$ ) the Gaussian beam would become a satisfactory solution of the Helmholtz equation, everywhere. This is not so: when $\psi_{G}$ is substituted into $k^{-2} \psi_{G}^{-1}$ times the Helmholtz equation, we obtain (|8|, Section 20.3), instead of zero, $\frac{2}{k^{2}(b+i z)}-$ $\frac{2 \rho^{2}}{k(b+i z)^{3}}+\frac{\rho^{4}}{4(b+i z)^{4}}$. It follows that the errors become small in regions where both of the following inequalities hold:

$$
k^{2}\left(b^{2}+z^{2}\right) \gg 1, \quad \text { and } b^{2}+z^{2} \gg \rho^{2} .
$$

Figure (3.1) shows the modulus and phase of $\psi_{G}$, for $k b=2$. Since the exponent of the modulus of $\psi_{G}$ tends to $-k b \rho^{2} / 2 z^{2}=-(k b) / 2 \tan ^{2} \theta$ far from the origin, the half-angle of the cone of divergence of the Gaussian beam, obtained by setting the exponent equal to -1 , is $\theta=\arctan \sqrt{2 / k b}$. The divergence angle defined in this way is $45^{\circ}$ for $k b=2$, and $30^{\circ}$ for $k b=6$.

Many authors $32,33,10,12,34,35,11,36$, have investigated methods to build up exact solutions of the Helmholtz equation from solutions of the paraxial equation, typically as expansions in powers of $(k b)^{-1}$ or of $w_{0} / b=\sqrt{2 / k b}$. 


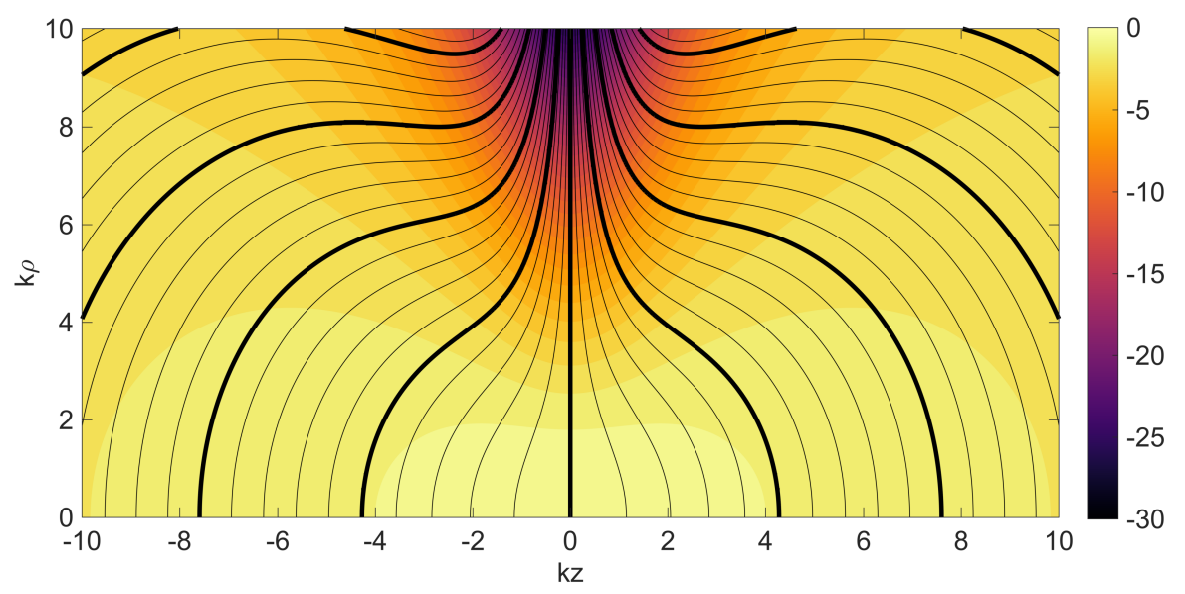

Figure 3.1: $\psi_{G}(\rho, z)$ in the focal region, plotted for $k b=2$, for $k|z| \leq 10, k \rho \leq$ 10. Shading indicates modulus of the wavefunction (logarithmic scale, lighter colour indicates larger modulus). The isophase surfaces are shown at intervals of $\pi / 3$. The phase is chosen to be zero at the origin. The isophase contours that are multiples of $\pi$ are drawn with heavier lines. The three-dimensional picture is obtained by rotating the figure about the beam axis (the horizontal axis).

These expansions have problems, not just in complexity, but in boundedness as well. A case in point is Wünsche's [12] operator method, which aims to get exact solutions from paraxial solutions by acting on the latter with differential operators (given as infinite series of partial derivatives with respect to $z$ ). We touch on this method in Appendix 3.9. Our aim here is different: we ask the question 'can any physical solution of the Helmholtz equation duplicate the Gaussian beam on the axis, or in the focal plane?' The answer to both questions is 'no', such solutions do not exist. (By 'physical' is meant causal and having finite beam invariants, as explained in the next Section.) This will be shown in Sections 3.4 and 3.5. We also show, in Appendix 3.8, that no Bessel-Gauss beam can be the same in its focal plane as an exact solution. But first we compare and contrast a recent exact solution with the Gaussian beam, in Sections 3.2 and 3.3 . 


\subsection{An exact solution and its properties}

A recent paper [14] discusses solutions of the Helmholtz equation (3.1.1) which represent transversely bounded beams, of the form

$$
\psi(\boldsymbol{r})=e^{i m \phi} \int_{0}^{k} \mathrm{~d} q f(k, q) J_{m}\left(\rho \sqrt{k^{2}-q^{2}}\right) e^{i q z} .
$$

Beams of this form propagate along the $z$ direction.

The wave motion is causal [14], meaning that far from the focal region there is no backward propagation. Ref. [14] discusses wavefunctions with no azimuthal dependence $(m=0)$, and gives an explicit expression for the case where $f(k, q)$ is proportional to $q$, in terms of Lommel functions of two variables, or equivalently in terms of products of spherical Bessel and Legendre functions. Proportionality to $q$ at small $q$ is sufficient to ensure the finiteness of beam invariants and of physical quantities such as the energy content per unit length of the beam ([14], Sections 5 and 6). Wavefunctions of the form (3.2.1) can all be generalized to

$$
\psi(\boldsymbol{r})=e^{i m \phi} \int_{0}^{k} \mathrm{~d} q f(k, q) J_{m}\left(\rho \sqrt{k^{2}-q^{2}}\right) e^{i q(z-i b)} .
$$

The imaginary translation in $z$, which leads to the extra factor $e^{q b}$ in the integrand, leaves the Laplacian unchanged, so (3.2.2) is still an exact solution of 3.1.1).

The $m=0$ beam with $f(k, q)$ set equal to a constant in 3.2.2) does approximate $\psi_{G}$ uniformly along the beam axis, with error of order $e^{-k b}$. However, $f(k, q)=$ constant is not physically possible: it does not give a finite energy content per unit length of the beam. For example, the corresponding transverse magnetic (TM) beam has energy content per unit length of the beam ([25], eqn. (26)) proportional to $\int_{0}^{k} \mathrm{~d} q q^{-1}\left(k^{2}-q^{2}\right) e^{2 q b}$, which diverges logarithmically.

As an example of a beam waveform which has all of the required physical properties, we shall consider the wavefunction of Section 9 of [14]:

$$
\psi_{b}(\rho, z)=\frac{b^{2}}{e^{k b}(k b-1)+1} \int_{0}^{k} \mathrm{~d} q q e^{q(b+i z)} J_{0}\left(\rho \sqrt{k^{2}-q^{2}}\right) .
$$




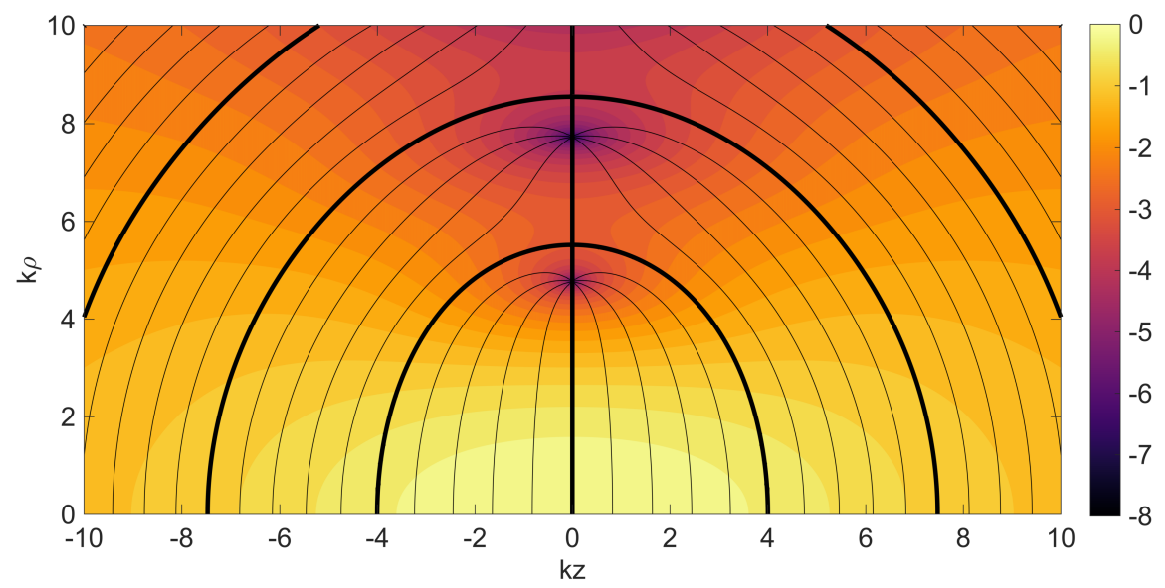

Figure 3.2: $\psi_{b}(\rho, z)$ in the focal region, plotted for $k b=2$, for $k|z| \leq 10, k \rho \leq$ 10. Shading indicates modulus of the wavefunction (logarithmic scale, lighter colour indicates larger modulus). The isophase surfaces are shown at intervals of $\pi / 3$. The phase is chosen to be zero at the origin. The isophase contours, other than those that are multiples of $\pi$, meet on the zeros of $\psi_{0}(\rho, z)$, three of which are shown, at $k \rho \approx 4.77,7.73$ and 10.77 .

The prefactor in 3.2.3) normalizes the wavefunction to unity at the origin $\rho=$ $0, z=0$, for easier comparison with $\psi_{G}$ given in 3.1 .2 , which is also normalized to unity at the origin.

We shall show that, for $e^{k b} \gg 1$ and $\rho^{2} \ll b / k$, where the Gaussian waveform has some validity, the wavefunction $\psi_{b}$ corresponds closely to it, provided that also $|z| \ll k b^{2}$. There are no constraints on where $\psi_{b}$ may be used, being an exact solution of (3.1.1). Figure 2 shows $\psi_{b}(\rho, z)$ in the focal region around the origin, for $k b$ again set equal to 2 .

On the beam axis $\rho=0$ we have

$$
\begin{aligned}
& \psi_{b}(0, z)=\frac{b^{2}}{e^{k b}(k b-1)+1} \int_{0}^{k} \mathrm{~d} q e^{q(b+i z)} \\
&=\left(\frac{b}{b+i z}\right) 2 \frac{e^{k(b+i z)}(k(b+i z)-1)+1}{e^{k b}(k b-1)+1} .
\end{aligned}
$$


An explicit form of $\psi_{b}$ at a general point $(\rho, z)$ was found in $[14 \mid$, using the fact that the expression 3.2 .3 is a cylindrically symmetric non-singular solution of the Helmholtz equation, and may thus be expanded as a sum over products of Legendre polynomials and spherical Bessels,

$$
\begin{aligned}
\psi_{b}(\rho, z)=\frac{(k b)^{2}}{e^{k b}(k b-1)+1} \sum_{n=0}^{\infty} a_{n} P_{n}\left(\frac{z-i b}{R}\right) j_{n}(k R), & \\
R & =(z-i b) \sqrt{1+\rho^{2} /(z-i b)^{2}} .
\end{aligned}
$$

As in Ref. [14], $R$ is chosen as a branch of the complex radial coordinate resulting from an imaginary displacement along the beam axis:

$$
r=\sqrt{\rho^{2}+z^{2}} \rightarrow R=\sqrt{\rho^{2}+(z-i b)^{2}} .
$$

The coefficients $a_{n}$ in the expansion are given in [14], Appendix B. There is only one non-zero odd coefficient, $a_{1}=2 i$. The even coefficients we shall rename as $a_{2} n=A_{n}$, so that

$$
\begin{aligned}
\psi_{b}(\rho, z)=\frac{(k b)^{2}}{e^{k b}(k b-1)+1}\left\{2 i P_{1}\left(\frac{z-i b}{R}\right)\right. & j_{1}(k R) \\
& \left.+\sum_{n=0}^{\infty} A_{n} P_{2 n}\left(\frac{z-i b}{R} j_{2 n}(k R)\right)\right\} .
\end{aligned}
$$

The full sequence of the coefficients of the even terms is (note that $(-1) ! !=1$ )

$$
\begin{aligned}
A_{0}=1, \quad A_{n} & =-\frac{(4 n+1)(2 n-3) ! !}{2^{n}(n+1) !} \\
& =-\frac{2(4 n+1)(2 n-3) ! !}{(2 n+2) ! !}, \quad n=1,2,3 \ldots
\end{aligned}
$$

At the origin $\rho=0, z=0$ we have $R \rightarrow-i b, P_{n}\left(\frac{z-i b}{R}\right) \rightarrow P_{n}(1)=1$, so (we set $k b=\beta$ )

$$
\psi_{b}(0,0)=\frac{\beta^{2}}{e^{\beta}(\beta-1)+1}\left\{2 i j_{1}(-i \beta)+\sum_{n=0}^{\infty} A_{n} j_{n}(-i \beta)\right\} .
$$

The right-hand side is unity because of the identities given in Eq. (9.6) of [14]. 
In the focal plane $z=0$ we have $R \rightarrow-i \sqrt{b^{2}-\rho^{2}}$, so

$$
\begin{aligned}
& \psi_{b}(\rho, 0)=\frac{\beta^{2}}{e^{\beta}(\beta-1)+1}\left\{2 i P_{i}\left(\frac{b}{\sqrt{b^{2}-\rho^{2}}}\right) j_{1}\left(-i k \sqrt{b^{2}-\rho^{2}}\right)\right. \\
&\left.+\sum_{n=0}^{\infty} A_{n} P_{2 n}\left(\frac{b}{\sqrt{b^{2}-\rho^{2}}}\right) j_{2 n}\left(-i k \sqrt{b^{2}-\rho^{2}}\right)\right\} .
\end{aligned}
$$

There is a removable singularity on the circle $\rho=b$ in the $z=0$ plane, since for large $X$ and small $x$

$$
P_{n}(X) \sim \frac{(2 n-1) ! !}{n !} X^{n}, \quad j_{n}(x) \sim \frac{x^{n}}{(2 n+1) ! !} .
$$

Thus as $\rho \rightarrow b$ the quantity in braces in 3.2 .10 tends to

$$
1+\frac{2 \beta}{3}+\sum_{n=1}^{\infty} A_{n} \frac{\left(-\beta^{2}\right)^{n}}{(4 n+1)(2 n) !}=1+\frac{2 \beta}{3}-\sum_{n=1}^{\infty} \frac{\left(-\beta^{2} / 4\right)^{n}}{n !(n+1) !(2 n-1)} .
$$

The final sum may be evaluated as

$$
\begin{aligned}
1-\frac{2}{3}\left[J_{0}(\beta)+\beta^{-1} J_{1}(\beta)\right]-\frac{2 \beta^{3}}{3}\left[J_{0}(\beta)\right. & \left.-\beta^{-1} J_{1}(\beta)\right] \\
+ & \frac{\pi \beta^{2}}{3}\left[J_{0}(\beta) H_{1}(\beta)-J_{1}(\beta) H_{0}(\beta)\right] .
\end{aligned}
$$

In this expression $J_{0}, J_{1}$ are the usual Bessel functions, and $H_{0}, H_{1}$ are the related Struve functions (Chapters 10 and 11 of $[6]$ ).

The focal plane wavefunction (3.2.10) is real for all $\rho$. It becomes oscillatory for $\rho>b$, in which case

$$
\begin{aligned}
\beta^{-2}\left[e^{\beta}(\beta-1)+1\right] \psi_{b}(\rho, 0)= & \frac{2 b}{\sqrt{\rho^{2}-b^{2}}} j_{1}\left(k \sqrt{\rho^{2}-b^{2}}\right) \\
& +\sum_{n=0}^{\infty} A_{n} P_{2 n}\left(\frac{-i b}{\sqrt{\rho^{2}-b^{2}}}\right) j_{n}\left(k \sqrt{\rho^{2}-b^{2}}\right)
\end{aligned}
$$

When $\rho^{2} \gg b^{2}$ the identity (B1) of $14 \mid$ shows that the right-hand side of 3.2.14 tends to $2 J_{1}(k \rho) / k \rho$. As expected, there is an infinite number of circles of wavefunction zeros in the focal plane, at which the different isophase surfaces from opposite sides of the focal plane can meet ( [8], Section 20.1.4).

The divergence angle of the beam is found in Appendix 3.7. For large $k b$ it is the same as for the Gaussian beam, $\theta \approx \sqrt{2 / k b}$. 


\subsection{The ratio of $\psi_{G}(\rho, z)$ to $\psi_{b}(\rho, z)$}

Both $\psi_{G}(\rho, z)$ and $\psi_{b}(\rho, z)$ are normalized to unity at the origin, chosen as the centre of the focal region. On the beam axis we have, and with $\beta=k b, \xi=k z$,

$$
\begin{aligned}
\psi_{G}(0, z) & =\left(\frac{\beta}{\beta+i \xi}\right) e^{i \xi} \\
\psi_{b}(0, z) & =\left(\frac{\beta}{\beta+i \xi}\right)^{2} \frac{(\beta+i \xi-1) e^{\beta+i \xi}+1}{(\beta-1) e^{\beta}+1} \\
\frac{\psi_{G}(0, z)}{\psi_{b}(0, z)} & =\frac{(\beta+i \xi) e^{i \xi}\left((\beta-1) e^{\beta}+1\right)}{\beta\left((\beta+i \xi-1) e^{\beta+i \xi}+1\right)}
\end{aligned}
$$

Thus the ratio of $\psi_{G}$ to $\psi_{b}$ on the axis is, for $\beta \gg 1$,

$$
\begin{aligned}
\frac{\psi_{G}(0, z)}{\psi_{b}(0, z)} & =\frac{(\beta+i \xi)(\beta-1)}{\beta(\beta+i \xi-1)}+\mathscr{O}\left(e^{-\beta}\right) \\
& =1-\frac{i \xi}{\beta^{2}}+\mathscr{O}\left(\beta^{-3}\right)+\mathscr{O}\left(e^{-\beta}\right)
\end{aligned}
$$

It follows that the ratio of the beam wavefunctions is close to unity when $\beta \gg 1$ and also $|z| \ll k b^{2}$.

We do not expect close correspondence far from the origin in the focal plane $z=0$, since the Gaussian beam has no zeros in the focal plane, while $\psi_{b}(\rho, 0)$ has an infinity of zeros, as we saw in the previous Section. Expansion of 3.2.10 in powers of $\rho$ gives

$$
\frac{\psi_{G}(\rho, 0)}{\psi_{b}(\rho, 0)}=1+\frac{1}{4} \frac{\left(2 \beta^{2}-4 \beta+4\right)+\beta^{2}-4}{e^{\beta}(\beta-1)+1} \frac{\rho^{2}}{b^{2}}+\mathscr{O}\left(\frac{\rho^{4}}{b^{4}}\right) .
$$

For $k b=\beta \gg 1$ the leading terms become $1+k \rho^{2} / 2 b$ (for $\beta \gg 1$ we find $1+$ $\rho^{2} / 2 b^{2}$ ). Thus there is close correspondence, in the focal plane, between the Gaussian beam and the exact solution if $k b \gg 1$ and $\rho^{2} \ll b / k$.

To sum up the correspondence between $\psi_{G}(\rho, z)$ and $\psi_{b}(\rho, z)$ : it is close within the region (surrounding the focus) defined by $\left\{|z| \ll k b^{2}, \rho^{2} \ll b / k\right\}$ provided $k b \gg 1$. Figure 3.3 shows the ratio of the moduli $\left|\psi_{G}(\rho, z) / \psi_{b}(\rho, z)\right|$ in the focal region, for $k b=2$. 


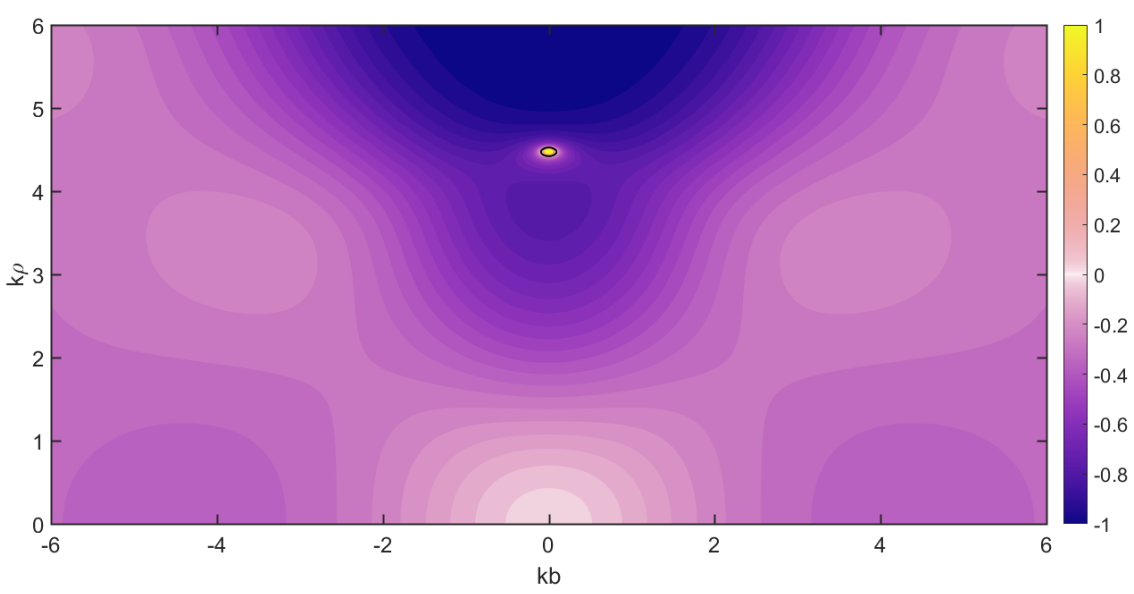

Figure 3.3: Ratio of the moduli $\left|\psi_{G}(\rho, z) / \psi_{b}(\rho, z)\right|$ in the focal region, plotted for $k b=2$. Shading indicates modulus of the ratio (colour bar indicates value of modulus ratio minus unity, so zero corresponds to the best agreement). The ratio tends to infinity at the zeros of $\psi_{b}(\rho, 0)$, one of which is shown, at $k \rho \approx$ 4.77 .

\subsection{Proof that an exact match to $\psi_{G}(0, z)$ does not exist}

It is known ([8], Section 20.1.1) that any solution of the Helmholtz equation 3.1.1) which is independent of the azimuthal angle may be written as

$$
\psi(\rho, z)=\frac{1}{2 \pi} \int_{0}^{2 \pi} d \theta g(z+i \rho \cos \theta) e^{-i k \rho \sin \theta} .
$$

On the beam axis $\psi(0, z)=g(z)$. Restricting ourselves to causally propagating beams (no backward propagation far from the focal region [14]) gives $\psi(\rho, z)$ of the form 3.2.2 with $m=0$. Equating the axial value to the Gaussian beam value, we have

$$
\psi(0, z)=g(z)=\int_{0}^{k} \mathrm{~d} q f(k, q) e^{q(b+i z)}=\frac{b}{b+i z} e^{i k z}
$$


The last equality is an integral equation for the function $f(k, q)$ (which could in addition depend on parameters such as the length $b$ ). Without loss of generality we may write $f(k, q)=b e^{-k b} h(k, q)$. Then

$$
\int_{0}^{k} \mathrm{~d} q h(k, q) e^{q(b+i z)}=\frac{e^{k(b+i z)}}{b+i z} .
$$

In terms of the dimensionless variables $\xi=k(b+i z), \eta=q(b+i z)$ this Volterra integral equation of the first kind becomes

$$
e^{\xi}=\int_{0}^{\xi} d \eta h\left(\frac{\xi}{b+i z}, \frac{\eta}{b+i z}\right) e^{\eta} .
$$

A solution independent of $z$ is required, so $h(k, q)$ has to be a function of one variable, the ratio $q / k$. Thus (3.4.4) reads

$$
e^{\xi}=\int_{0}^{\xi} h\left(\frac{\eta}{\xi}\right) e^{\eta} \text {, or, with } \eta=\xi t, \frac{e^{\xi}}{\xi}=\int_{0}^{1} d t h(t) e^{\xi t} .
$$

We shall argue that (3.4.5) has no physical solution. The function $h(t)$ would have to be such that $\int_{0}^{1} d t h(t)$ diverges, while $\int_{0}^{1} d t h(t) e^{\xi t}$ does not, for any $\xi \neq 0$. This makes a physical solution unlikely. To proceed further we note that $\xi$ is a complex variable, and that the left-hand side of the last equation has a simple pole at the origin. The contour integral around the origin of the complex $\xi$ plane gives

$$
\oint d \xi \frac{e^{\xi}}{\xi}=2 \pi i
$$

As long as $h(t)$ is smooth enough (which is needed for a physical beam with finite invariants), we can change the order of integration in the double integral on the right-hand side:

$$
\oint d \xi \int_{0}^{1} d t h(t) e^{\xi t}=\int_{0}^{1} d t h(t) \oint d \xi e^{\xi t}=\int_{0}^{1} d t h(t) 0=0 .
$$

We have thus reached a contradiction, which implies that no solution of the required form exists.

The same conclusion follows by considering the Laplace transform of equation 3.4.5 at $z=0$, written in the form $e^{\beta}=\beta \int_{0}^{1} d t h(t) e^{\beta t}, \beta=k b$. Taking 
Laplace transforms with respect to $\beta$ of both sides, we obtain, for $s>1$,

$$
\begin{aligned}
\int_{0}^{\infty} \mathrm{d} \beta e^{\beta(1-s)} & =\int_{0}^{1} d t h(t) \int_{0}^{\infty} \mathrm{d} \beta e^{\beta(t-s)}, \\
\frac{1}{1-s} & =\int_{0}^{1} d t \frac{h(t)}{(s-t)^{2}} .
\end{aligned}
$$

The leading asymptotic terms for large $s$ are $s^{-1}$ on the left-hand side, and $s^{-2}$ on the right- hand side. Again we have reached a contradiction.

\subsection{An exact solution with Gaussian profile in the fo- cal plane does not exist}

The general form of a causally propagating cylindrically symmetric beam is (on changing the variable of integration in 3.2.1 from $q$ to $\kappa=\sqrt{k^{2}-q^{2}}$

$$
\psi(\rho, z)=\int_{0}^{k} \mathrm{~d} \kappa \kappa h(k, \kappa) e^{i z \sqrt{k^{2}-\kappa^{2}}} J_{0}(\kappa \rho) .
$$

The extra factor of $\kappa$ has been inserted for convenience in the application of Hankel transforms. In the focal plane $z=0$ this is to be of Gaussian form:

$$
\psi(\rho, 0)=\int_{0}^{k} \mathrm{~d} \kappa \kappa h(k, \kappa) J_{0}(\kappa \rho)=\exp \left(-\frac{k \rho^{2}}{2 b}\right) .
$$

Taking the Hankel transform of (3.5.2) by operating with $\int_{0}^{\infty} d \rho \rho J_{0}\left(\kappa^{\prime} \rho\right)$, we get

$$
\int_{0}^{k} \mathrm{~d} \kappa \kappa h(k, \kappa) \int_{0}^{\infty} d \rho \rho J_{0}\left(\kappa^{\prime} \rho\right) J_{0}(\kappa \rho)=\int_{0}^{\infty} d \rho \rho J_{0}\left(\kappa^{\prime} \rho\right) \exp \left(-\frac{k \rho^{2}}{2 b}\right)
$$

The Hankel transform of a Gaussian is proportional to a Gaussian (formula 10.22.51 of $[6])$,

$$
\int_{0}^{\infty} d \rho \rho J_{0}(\kappa \rho) \exp \left(-\frac{k \rho^{2}}{2 b}\right)=\frac{b}{k} \exp \left(-\frac{b \kappa^{2}}{2 k}\right) .
$$

The Hankel transform of a Bessel function is proportional to a Dirac delta,

$$
\int_{0}^{\infty} d \rho \rho J_{0}\left(\kappa^{\prime} \rho\right) J_{0}(\kappa \rho)=\kappa^{-1} \delta\left(\kappa^{\prime}-\kappa\right) .
$$


Let $U(k, \kappa))$ be unity for $0 \leq \kappa \leq k$, and zero otherwise. Thus 3.5.3 reads, on dropping the prime on $\kappa^{\prime}$,

$$
U(k, \kappa) h(k, \kappa)=\frac{b}{k} \exp \left(-\frac{b \kappa^{2}}{2 k}\right)
$$

The left-hand side is nonzero only in the interval $0 \leq \kappa \leq k$, while the right-hand side is nonzero on the entire positive real line. Thus there is no solution. To have a causal and non-divergent solution of the Helmholtz equation, we restrict the range in (3.5.1) to the interval $[0, k]$, but the resultant Hankel transform is not that of a Gaussian.

\subsection{Discussion}

The 'paraxial' Gaussian beam waveform (3.1.2), and its generalizations, the LaguerreGauss and Hermite-Gauss waveforms, have been widely used. However, they are known to fail for strongly focused beams. It is interesting that, despite 3.1.2 being the solution of a paraxial equation, and thus presumed to be accurate close to the beam axis, the fact is that no exact solution of the Helmholtz equation can reproduce the Gaussian beam waveform on the axis. Thus the 'paraxial' Gaussian beam cannot give an exact representation of physical beams, even on the beam axis. It does remain a useful approximation within the focal region provided $b^{2}+z^{2} \gg \rho^{2}$ and $k^{2}\left(b^{2}+z^{2}\right) \gg 1$, as we saw in Section 3.1. (These inequalities are satisfied when $k b \gg 1, \rho \ll \sqrt{k / b},|z| \ll k b^{2}$, in accord with the results of Section 3.3 .)

In Appendix 3.8 we show that the simplest Bessel-Gauss beam also cannot be the basis of an exact solution. This is more surprising, since the Bessel-Gauss beam does have the expected zeros in the focal plane.

In Appendix 3.9 we discuss the transformation of solutions of the paraxial equation into solutions of the Helmholtz equation, in parallel to a method proposed by Wünsche [12]. We show that the resultant 'solutions' have nonphysical divergences. 
As regards the exact solution $\psi_{b}(\rho, z)$, we note that, by construction, it contains no 'evanescent' waves (namely those decaying or growing exponentially with $z$ ). This is because the variable $q$ which appears as $e^{i q z}$ in the integrand defining $\psi_{b}(\rho, z)$ is real in its entire range. The absence of evanescent waves is in contrast to much literature on beams in which $q=k_{z}=\sqrt{k^{2}-k_{x}^{2}-k_{y}^{2}}$ is allowed to be imaginary, leading to exponential growth or decay. Exponential growth with $z$ is not physical. It can be avoided by choosing different branches of the square root for positive and negative $\mathrm{z}$, or equivalently by replacing $e^{i q z}$ by $e^{i q|z|}$. However, this leads to backward propagation for negative $z$, and the resulting wavefunction is no longer a solution of the Helmholtz equation at $z=0$, because the derivative of $|z|$ is a step function, and the second derivative a delta function. Evanescent waves exist, for example, within the medium of smaller refractive index in total reflection, but have no part in free space propagation far from material boundaries, in our view.

\subsection{Divergence angle of the $\psi_{b}(\rho, z)$ beam}

We wish to examine how the beam expands away from the axis. The behaviour is complicated within the focal region, but simplifies when $|z| \gg b, r \gg b$ and $k r \gg 1$. Let us find the asymptotic form of the un-normalized wavefunction, which from 3.2 .7 is

$$
\Psi(\rho, z)=2 i P_{1}\left(\frac{z-i b}{R}\right) j_{1}(k R)+\sum_{n=0}^{\infty} A_{n} P_{2} n\left(\frac{z-i b}{R}\right) j_{2 n}(k R) .
$$

Since $R^{2}=r^{2}-2 i b z+b^{2}$, we have $R \approx r-i b z / r$. The argument of the Legendre polynomials is therefore $z / r=\cos \theta$ plus correction terms of order $b z$ and $b z r^{2}$, which we can neglect. However, $k R \approx k r-i k b z / r$, and the imaginary part is important because it leads to hyperbolic terms in the spherical Bessel functions, for which the asymptotic forms for large $|\zeta|$ are

$$
j_{1}(\zeta) \rightarrow-\frac{\cos \zeta}{\zeta}, \quad j_{2 n}(\zeta) \rightarrow(-)^{n} \frac{\sin \zeta}{\zeta}
$$


We also know from equation (3.20) of $|\overline{8}|$ that

$$
\sum_{n=0}^{\infty}(-)^{n} A_{n} P_{2 n}(\cos \theta)=2|\cos \theta| .
$$

Hence the asymptotic form of 3.7 .1 is, on setting $k R \approx k r-i k b \cos \theta$,

$$
\begin{aligned}
\frac{2}{k r}(-i \cos \theta \cos (k r-i k b \cos \theta)+|\cos \theta| \sin (k r-i k b \cos \theta)) \\
=-\frac{2 i|\cos \theta|}{k r} \exp (i k r \operatorname{sgn}(\cos \theta)+k b|\cos \theta|) .
\end{aligned}
$$

The asymptotic ratio of the moduli (off-axis to on-axis) of $\Psi$ is therefore

$$
|\Psi(\rho, z) / \Psi(0, z)| \rightarrow \cos ^{2} \theta e^{-k b(1-|\cos \theta|)} .
$$

When $k b$ is large compared to unity the exponent in 3.7 .5 is -1 when $\theta \approx$ $\sqrt{2 / k b}$, which is the same as for the Gaussian beam for large $k b$, given below equation (3.1.4). For comparison, for $\psi_{G}$ the asymptotic ratio of the moduli (off-axis to on-axis) is $e^{-k b / 2 \tan ^{2} \theta}$, quite different from (3.7.5) except at small $\theta$.

\subsection{An exact solution reproducing the simplest Bessel- Gauss beam in the focal plane does not exist}

Bessel-Gauss beams, in their simplest azimuthally symmetric form, are defined by the focal plane wavefunction being set equal to that of the Gaussian beam times a Bessel function of order zero [37]:

$$
\psi(\rho, 0)=e^{-k \rho^{2} / 2 b} J_{0}(k \rho) .
$$

Comparison with exact solutions of the form 3.5.1) gives the equality, on $z=0$,

$$
\psi(\rho, 0)=\int_{0}^{k} \mathrm{~d} \kappa \kappa h(k, \kappa) J_{0}(\kappa \rho)=e^{-k \rho^{2} / 2 b} J_{0}(\kappa \rho) .
$$

The function $h(k, \kappa)$ is to be found, if possible. Let $U(k, \kappa)$ be unity for $0 \leq \kappa \leq k$, and zero otherwise. Then (3.8.2) is the Hankel transform of $U(k, \kappa) h(k, \kappa)$ :

$$
\psi(\rho, 0)=\int_{0}^{\infty} \mathrm{d} \kappa \kappa U(k, \kappa) h(k, \kappa) J_{0}(\kappa \rho) .
$$


Taking the inverse Hankel transform of (3.8.2) by operating with $\int_{0}^{\infty} d \rho \rho J_{0}\left(\kappa^{\prime} \rho\right)$, and using (3.5.5), we get (dropping the primes on $\kappa$ )

$$
U(k, \kappa) h(k, \kappa)=\int_{0}^{\infty} d \rho \rho e^{-k \rho^{2} / 2 b} J_{0}(\kappa \rho) J_{0}(k \rho) .
$$

The right-hand side of (3.8.4) is a special case of Weber's second exponential integral ( [38], Section 13.31), and is equal to

$$
\frac{b}{k} \exp \left(\frac{b\left(k^{2}+\kappa^{2}\right)}{2 k}\right) I_{0}(\kappa b)
$$

Thus we again have a contradiction, since $U(k, \kappa) h(k, \kappa)$ is nonzero only when $0 \leq \kappa \leq k$, while the expression 3.8.5 is not.

\subsection{Converting solutions of the paraxial equation into solutions of the Helmholtz equation}

Wünsche [12] has found operators which formally convert solutions of the paraxial equation into solutions of the Helmholtz equation. We shall give an equivalent method here, and at the same time demonstrate the weakness of such constructions.

The (approximate) paraxial equation is, from Section 3.1 and for axiallysymmetric solutions,

$$
\left(\partial_{\rho}^{2}+\rho^{-1} \partial_{\rho}+2 i k \partial_{z}\right) G \approx 0, \quad \psi(\rho, z)=e^{i k z} G(\rho, z) .
$$

The paraxial equation is solved by $G=e^{-i q z} J_{0}(\rho \sqrt{2 k q})$ for any q. Superposition with the weight function $k^{-1} g(k, q)$ gives the general (regular) paraxial solution,

$$
G(\rho, z)=k^{-1} \int_{0}^{\infty} \mathrm{d} q g(k, q) e^{-i q z} J_{0}(\rho \sqrt{2 k q}) .
$$

The substitution $q=\kappa^{2} / 2 k$ gives us

$$
G(\rho, z)=k^{-2} \int_{0}^{\infty} \mathrm{d} \kappa \kappa g\left(k, \kappa^{2} / 2 k\right) e^{-i z \kappa^{2} / 2 k} J_{0}(\kappa \rho) .
$$


As an example, let us match (3.9.3) to the Gaussian beam (3.1.2), for which

$$
G(\rho, z)=\frac{b}{b+i z} \exp \left(-\frac{k \rho^{2}}{2(b+i z)}\right) \text {. }
$$

To find the corresponding function $g$, we take the Hankel transform of 3.9.4 equated to 3.9.3), using (3.5.4) and (3.5.5): operation with $\int_{0}^{\infty} d \rho \rho J_{0}\left(\kappa^{\prime} \rho\right)$ gives

$$
\frac{b}{k} \exp \left(-\frac{\kappa^{\prime 2}(b+i z)}{2 k}\right)=k^{-2} g\left(k, \kappa^{\prime 2} / 2 k\right) \exp \left(-\frac{i z \kappa^{\prime 2}}{2 k}\right) .
$$

Hence the weight function corresponding to the fundamental Gaussian mode is $g\left(k, \kappa^{2} / 2 k\right)=k b e^{-b \kappa^{2} / 2 k}$; substitution in (3.9.3) gives

$$
G=b /(b+i z) \exp \left(-\left(k \rho^{2}\right) / 2(b+i z)\right)
$$

Note there is no cut-off in the integration at $\kappa=k$. As in Section 3.5, such a weight function leads to an exact solution of the Helmholtz equation of the form

$$
\psi(\rho, z)=\frac{b}{k} \int_{0}^{\infty} \mathrm{d} \kappa \kappa \exp \left(i z \sqrt{k^{2}-\kappa^{2}-\frac{b \kappa^{2}}{2 k}}\right) J_{0}(\kappa \rho)
$$

This integral reproduces the Gaussian beam waveform in the focal plane. On the beam axis the integral can be evaluated in terms of error functions. We find that it grows without bound on the beam axis for negative $z$ large compared to $\sqrt{2 b / k}$, in proportion to $z e^{k z^{2} / 2 b}$. Thus 3.9.7) is not a physical wavefunction. On the other hand, if the integral in 3.9.7) is terminated at $\kappa=k$, the resulting wavefunction is a well-behaved member of the generalized Bessel beam family [14], and in fact reduces to the proto-beam of [14] when $k b \rightarrow 0$.

The same problem consequent (in our approach) from infinite range in the integral, arises in Wünsche's method of converting solutions of the paraxial equation into solutions of the Helmholtz equation, but in another guise. In evaluating the correction to the Gaussian beam on the beam axis, Wünsche ([12], Section 5 and Appendix B) also finds the error functions which result from setting $\rho=0$ in (3.9.7). 


\section{Chapter 4}

\section{Topology of phase and polarisation singularities in focal regions}

The following chapter includes verbatim a paper we have co-authored with John Lekner, published in Journal of Optics [23]. This paper was produced as part of this thesis project, and discusses the peculiar behaviour of the zeros of a particular wavefunction. This wavefunction, which we refer to as the 'Carter' wavefunction, is the closest approximation to a Gaussian beam radially, and like the Gaussian beam is controlled by a single parameter $k b$. For $k b=0$ this wavefunction becomes the proto-beam, and has an infinite set of zeros in the focal plane. At critical values of $k b$ neighbouring pairs of zeros annihilate, and a new pair is created off-plane. We construct electromagnetic beams from this wavefunction, and show that the behaviour of these zeros are associated with creation and destruction of polarisation singularities. We derive an equation that may be solved numerically to obtain the exact focal parameter at which the focal transitions occur. 


\section{Abstract}

The focal region of a beam contains circles of zeros of the beam wavefunction, on which surfaces of different phase meet. The existence of these zeros is topological in origin, and appears to be universal. Two examples of generalized Bessel beams are examined. One of these has zeros only in the focal plane. The other has focal plane zeros but also movement of the zeros away from the focal plane at certain values of a parameter which determines the tightness of the focus, as analysed by Berry in 1998. As tightness of focus increases these two families of beams coalesce into a common most-focused beam. The polarization properties of both families and of their common limiting form are considered and correlated with the zeros (dislocations) of the beam wavefunctions. We find regions of circular polarization in beams which are nominally linearly polarized, and rapid variation of the polarization pattern as the tightness of focus passes through critical values.

\subsection{Introduction}

The focal regions of beams (optical or acoustic) are the most interesting, experimentally and theoretically. A key property of the focal region is that it is where the wavefronts converge onto, and diverge from. In a monochromatic beam of angular frequency $\omega=c k$, the beam wavefunction carries an overall phase factor $e^{-i \omega t}$, and a local phase $P(\boldsymbol{r})$. The isophase surfaces $P(\boldsymbol{r})=$ constant are the wavefronts (in an electromagnetic beam each component of $\boldsymbol{E}$ and $\boldsymbol{B}$ has, in general, its own set of wavefronts). The phase of a complex wavefunction is not defined at a zero of the wavefunction, where both the real and the imaginary parts are zero. This is where different isophase surfaces can meet. As time advances the surfaces on which $P(\boldsymbol{r})-\omega t=$ constant rotate about the complex zeros of the beam wavefunction, and some authors call the zeros optical vortices. 
Nye and Berry [39] refer to these wavefunction zeros as wave dislocations. They are discussed in Nye's book [40], but the emphasis there is on natural focusing, as in the earlier studies of polarizations singularities by Berry and Dennis 41 42, 43]. The emphasis in these papers is, like Nye's [40], mainly on properties 'in the wild' [42], random or disordered fields. The focal region, in contrast, is highly structured and symmetric.

Here we are interested in polarization near the focus of a light beam. Karman et al [44, 17] have shown experimentally and theoretically that phase singularities in the focal region can be created and annihilated, and can move off the focal plane. An earlier numerical study by Carter [15] reported the same behaviour. Berry [16] and Nye [45] have given thorough discussions of this phenomenon; we shall follow Berry's classification here. Structured light itself is the subject of a recent roadmap [46], of which part 2 is a review of 'Vortices, natural and deliberate' by Berry and Dennis.

We shall discuss properties of exact ('non-paraxial') solutions of the Helmholtz equation $\left(\nabla^{2}+k^{2}\right) \psi=0$, which in cylindrical coordinates $(\rho, \phi, z)$ reads

$$
\left(\partial_{z}^{2}+\rho^{-1} \partial_{\rho}+\rho^{-2} \partial_{\phi}^{2}+\partial_{z}^{2}\right) \psi=0
$$

The Helmholtz equation is solved by $J_{0}(\kappa \rho) e^{i m \phi} e^{i q z}$ provided that the transverse and longitudinal wavenumber components $\kappa$ and $q$ are constrained by $\kappa^{2}+q^{2}=k^{2}$. Superpositions of such solutions with a weight function give generalized Bessel beams [14],

$$
\psi(\rho, \phi, z)=e^{i m \phi} \int_{0}^{k} \mathrm{~d} \kappa f(k, \kappa) J_{m}(\kappa \rho) e^{i q z}, \quad \kappa^{2}+q^{2}=k^{2} .
$$

We shall be considering beams with no azimuthal dependence, $m=0$. The beam studied by Carter [15] and by Berry [16] is of the generalized Bessel beam form,

$$
\psi_{C}(\rho, z)=\frac{b}{k}\left(1-e^{-\frac{k b}{2}}\right)^{-1} \int_{0}^{k} \mathrm{~d} \kappa \kappa e^{-\frac{b \kappa^{2}}{2 k}+i q z} J_{0}(\kappa \rho) .
$$

We have normalized the beam wavefunction to unity at the origin, the centre of the focal region. The (positive) length $b$ and the wavenumber $k=\omega / c$ 
together form the dimensionless parameter $k b$ which characterises the beam. The weight factor is related to the Gaussian beam, an approximate (paraxial) solution of (4.1.1): the integral in 4.1.3) extended to the range $0 \leq \kappa<\infty$ reproduces, in the focal plane, the paraxial Gaussian fundamental mode, $\psi_{G}(\rho, z)=$ $\frac{b}{b+i z} e^{i k z-k \rho^{2} / 2(b+i z)}$ (see [22], Appendix C). However, it is shown in [22] that an exact solution of the Helmholtz equation which agrees with $\psi_{G}$, either in the focal plane or on the beam axis, does not exist.

The wavefunction (4.1.3) shows the phenomena mentioned above, of phase singularities in the focal region being created and annihilated, and moving off the focal plane. In contrast, the wavefunction [14

$$
\psi_{b}(\rho, z)=\frac{b^{2}}{e^{k b}(k b-1)+1} \int_{0}^{k} \mathrm{~d} \kappa \kappa e^{q(b+i z)} J_{0}(\kappa \rho) .
$$

has all of its zeros in the focal plane. (Again, the prefactor ensures that the wavefunction is normalized to unity at the origin, $\psi_{b}(0,0)=1$.) As the dimensionless parameter $k b$ tends to zero, both 4.1.3 and (4.1.4) tend to the proto-beam of [14],

$$
\begin{aligned}
\psi_{0}(\rho, z) & =\frac{2}{k^{2}} \int_{0}^{k} \mathrm{~d} \kappa \kappa e^{i q z} J_{0}(\kappa \rho) \\
& =\frac{2}{k^{2}} \int_{0}^{k} \mathrm{~d} q q e^{i q z} J_{0}(\kappa \rho) .
\end{aligned}
$$

The proto-beam is the most tightly focused confluent limit of the two beam families 4.1.3) and 4.1.4). Only one length $\left(k^{-1}\right)$ enters into its definition, and indeed the extent of its focal region, both longitudinally and transversely, is of order $k^{-1}$ ( |14|, Section 8). The proto-beam has all of its zeros in the focal plane, where $\psi_{0}(\rho, 0)=2 J_{1}(k \rho / k \rho)$.

In this paper we shall compare and contrast the two beam families 4.1.3 and (4.1.4), both characterized by the tightness of focus parameter $k b$. We begin in Sections 4.2 and 4.3 with the isophase surfaces and loci of the wavefunction zeros as functions of $k b$. Sections 4.4 and 4.5 discuss the polarization of the electric field in beams based on these two families and on their confluent tight-focus form. 


\subsection{Properties of the beam wavefunction $\psi_{b}(\rho, z)$}

On the beam axis $\rho=0$ the wavefunction (4.1.4 becomes

$$
\begin{aligned}
\psi_{b}(0, z) & =\frac{b^{2}}{e^{k b}(k b-1)+1} \int_{0}^{k} \mathrm{~d} q q e^{q(b+i z)} \\
& =\left(\frac{b}{b+i z}\right)^{2} \frac{e^{k(b+i z)}(k(b+i z)-1)+1}{e^{k b}(k b-1)+1}
\end{aligned}
$$

$\psi_{b}(0, z)$ behaves asymptotically as a constant times $\left(k z^{-1} e^{i k z}\right)$. There are no wavefunction zeros on the beam axis.

Figure 4.1 shows the isophase surfaces of $\psi_{b}$. All of the zeros of $\psi_{b}$ lie in the focal plane $z=0$, and so the surfaces of negative phase from the $z<0$ half-plane meet the surfaces of positive phase from the $z>0$ half-plane on the focal plane, for all values of $k b$. The three-dimensional picture is obtained by rotating the figure about the beam (horizontal) axis.

Figure 4.2 shows how the focal plane zeros of $\psi_{b}$ vary with the parameter $k b$. (The length determines the extent of the focal region: for large $k b$ it gives the longitudinal extent, $\sqrt{2 b / k}$ gives the beam waist in the focal plane, and $\sqrt{2 / k b}$ is the divergence angle of the beam far from focus as shown in [22], Appendix A.) We see from the figure that the zeros move outward from the beam axis as $k b$ increases.

\subsection{Properties of the Carter beam wavefunction}

The Carter wavefunction shows topologically different behaviour, except at small $k b$. On the beam axis the Carter wavefunction 4.1.3 becomes

$$
\psi_{C}(0, z)=\frac{b}{k}\left(e^{\frac{k b}{2}}-1\right)^{-1} \int_{0}^{k} \mathrm{~d} q q e^{\frac{b q^{2}}{2 k}+i q z}
$$

The integral may be evaluated in terms of error functions. It behaves asymptotically as a constant times $(k z)^{-1} e^{i k z}$. There are no wavefunction zeros on the beam axis. 


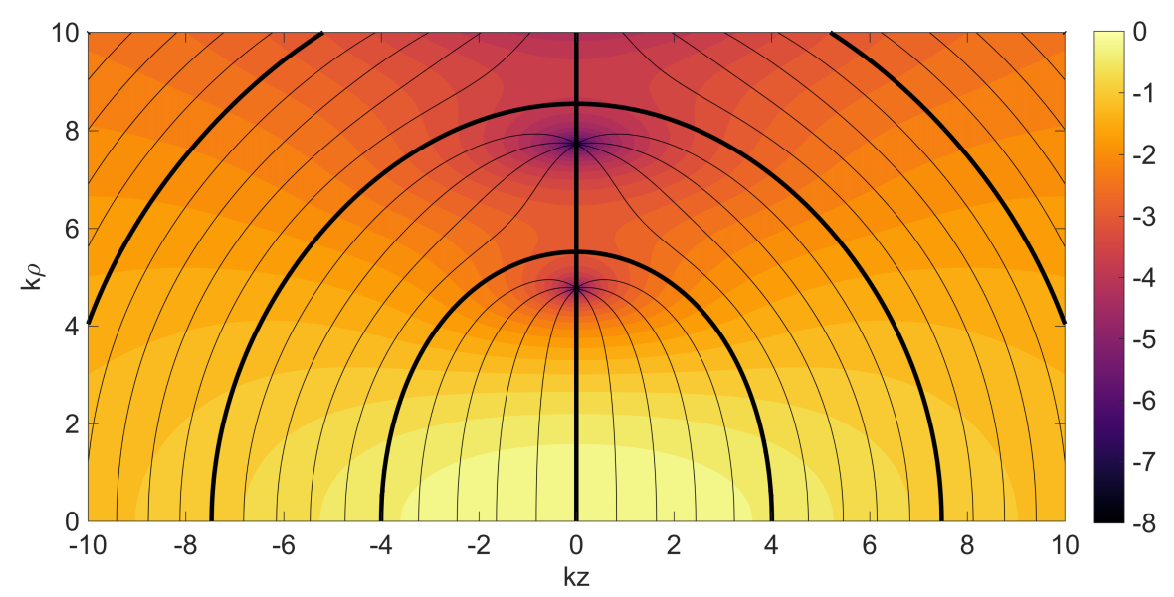

Figure 4.1: $\psi_{b}(\rho, z)$ in the focal region, plotted for $k b=2$, for $k|z| \leq 10, k \rho \leq$ 10. Shading indicates modulus of the wavefunction (logarithmic scale, lighter colour indicates larger modulus). The isophase surfaces are shown at intervals of $\pi / 3$. The phase is chosen to be zero at the origin. The isophase contours, other than those that are multiples of $\pi$, meet on the zeros of $\psi_{b}(\rho, z)$, three of which are shown, at $k \rho \approx 4.77,7.73$ and 10.77 .

Figure 4.3 shows the curves on which the real and imaginary parts of the wavefunction $\psi_{C}(\rho, z)$ are zero, in the $k z, k \rho$ plane. Where they meet gives the location of the complex zeros of $\psi_{C}$, which lie on circles centred on the beam axis.

In the six diagrams shown we transition from all complex zeros on the focal plane (up to $k b=4.91961)$, to on-plane plus off-plane zeros $(k b=5.15279$ to $k b=5.25477$ ), beyond which the innermost focal region shown has off-plane zeros only.

Berry $\left[16 \mid\right.$ has identified three topological events in $\psi_{C}$ as the focal tightness parameter $k b$ increases $\left(k b \rightarrow 0\right.$ gives the tightest focus, the confluence of $\psi_{b}$ and $\psi_{C}$ into the proto-beam $\psi_{0}$ ). These are events that involve the phase dislocations, where $\psi=0$, and phase saddles, where $\nabla P=0$. The phase saddles or stagnation points can be seen clearly on figure 4.1 , they occur between the 
zeros at circles where the integer $\pi$ phases (thicker isophase curves) intersect the focal plane. The topological events in $\psi_{C}$ are:

- Event $a$, at $k b=4.91961$. Lower saddle passes through upper zero.

- Event $b$, at $k b=5.15279$. Lower saddle collides with upper saddle.

- Event $c$, at $k b=5.25477$. Lowest two zeros coalesce.

The zeros in the focal plane are zeros of the function

$$
k^{-2} \int_{0}^{k} \mathrm{~d} \kappa \kappa e^{-\frac{b \kappa^{2}}{2 k}} J_{0}(\kappa \rho)=\int_{0}^{1} d \xi \xi e^{-(k b / 2) \xi^{2}} J_{0}(k \rho \xi)=F(k \rho, k b) .
$$

The saddle points in the focal plane are at zeros of the function

$$
\begin{aligned}
k^{-3} \int_{0}^{k} \mathrm{~d} \kappa \kappa \sqrt{k^{2}-\kappa^{2}} e^{-\frac{b \kappa^{2}}{2 k}} J_{0}(\kappa \rho)= & \int_{0}^{1} d \xi \xi \sqrt{1-\xi^{2}} \\
& \times e^{-(k b / 2) \xi^{2}} J_{0}(k \rho, \xi)=G(k \rho, k b) .
\end{aligned}
$$

Event $a$ corresponds to $F(k \rho, k b)=0=G(k \rho, k b)$. The first three numerical values of $(k \rho, k b)=\left(\alpha_{n}, \beta_{n}\right)$ at which this happens are (to five digit accuracy)

$$
\begin{aligned}
& \alpha_{1}=7.4395, \quad \alpha_{2}=13.843, \quad \alpha_{3}=20.183 \text {, } \\
& \beta_{1}=4.9196, \quad \beta_{2}=10.634, \quad \beta_{3}=16.549 \text {. }
\end{aligned}
$$

Event $b$ corresponds to $G(k \rho, k b)=0=\partial_{\rho} G(k \rho, k b)$. The first three numerical values of $(k \rho, k b)=\left(\alpha_{n}, \beta_{n}\right)$ at which this happens are

$$
\begin{array}{lll}
\alpha_{1}=8.0277, & \alpha_{2}=14.401, & \alpha_{3}=20.721, \\
\beta_{1}=5.1528, & \beta_{2}=10.856, & \beta_{3}=16.760 .
\end{array}
$$

Event $c$ corresponds to $F(k \rho, k b)=0=\partial_{\rho} F(k \rho, k b)$. The first three numerical values of $(k \rho, k b)=\left(\alpha_{n}, \beta_{n}\right)$ at which this happens are

$$
\begin{array}{lll}
\alpha_{1}=7.0156, & \alpha_{2}=13.324, & \alpha_{3}=19.616, \\
\beta_{1}=5.2548, & \beta_{2}=11.206, & \beta_{3}=17.287 .
\end{array}
$$

The numerical values for agree in each case with those listed in the penultimate row of table 1 of Berry [16], with the parameter $L$ corresponding to our $k b$. 
Figure 4.4 shows the zeros of the Carter wavefunction in the focal plane. As $k b$ increases from zero the null curves initially move further away from the beam axis $\rho=0$, but not as rapidly as for $\psi_{b}$ in figure 4.2. However, they eventually curve towards each other, and meet in pairs. The topological events identified by Berry are shown as points on this graph. It is interesting that for $k b<4.91961$ all the zeros lie on the focal plane; it is only as we loosen the focus that the off-plane zeros appear.

\subsection{Polarization properties of TM, TE and 'LP' beams}

We shall work with complex vector fields $\boldsymbol{A}(\boldsymbol{r}), \boldsymbol{B}(\boldsymbol{r}), \boldsymbol{E}(\boldsymbol{r})$; the real physical fields are obtained by taking, for example, the real part of $\boldsymbol{E}(\boldsymbol{r}) e^{-i \omega t}$, which is $\boldsymbol{E}_{r}(\boldsymbol{r}) \cos \omega t+\boldsymbol{E}_{i}(\boldsymbol{r}) \sin \omega t$. A measure of the degree of linear polarization of the electric field is given by 20

$$
\Lambda(\boldsymbol{r})=\frac{\left(\left(E_{r}^{2}-E_{i}^{2}\right)^{2}+4\left(\boldsymbol{E}_{r} \cdot \boldsymbol{E}_{i}\right)^{2}\right)^{\frac{1}{2}}}{E_{r}^{2}+E_{i}^{2}}=\frac{\left|\boldsymbol{E}^{2}(\boldsymbol{r})\right|}{|\boldsymbol{E}(\boldsymbol{r})|^{2}} .
$$

$\Lambda(\boldsymbol{r})$ is unity when the real and imaginary parts of $\boldsymbol{E}(\boldsymbol{r})=\boldsymbol{E}_{r}(\boldsymbol{r})+i \boldsymbol{E}_{i}(\boldsymbol{r})$ are collinear (the linear polarisation condition), and zero when the circular polarisation conditions $\boldsymbol{E}_{r} \cdot \boldsymbol{E}_{i}=0, E_{r}^{2}=E_{i}^{2}$ are met. The complex electric field $\boldsymbol{E}(\boldsymbol{r})$ is orthogonal to itself on these $C$ lines $[46 \mid: \boldsymbol{E}(\boldsymbol{r}) \cdot \boldsymbol{E}(\boldsymbol{r})=0$. Explicit expressions for $\Lambda(\boldsymbol{r})$ in terms of the derivatives of $\psi$ with respect to $\rho$ and $z$ when $\psi$ is independent of the azimuthal angle $\phi$ are given in $|20|$. The function $\Lambda(\boldsymbol{r})$ is related to the Stokes parameters ( $|19|$, Sections 1.4 .2 and 10.8.3) by $\Lambda=\sqrt{1-S_{3}^{2} / S_{0}^{2}}$, and to the Hurwitz [47] polarization parameter $S$ by $S^{2}+\Lambda^{2}=1$.

The simplest example of pure linear polarization is the TM (transverse magnetic) beam, for which the vector potential is given by $\boldsymbol{A}=A_{0}[0,0, \psi]$. (See for example [20]; square brackets denote Cartesian coordinates.) For this beam the magnetic field $\boldsymbol{B}$ is everywhere transverse to the propagation direction, here along the $z$ axis:

$$
\boldsymbol{B}=\nabla \times \boldsymbol{A}=A_{0}\left[\partial_{y},-\partial_{x}, 0\right] \psi
$$


When $\psi$ is independent of the azimuthal angle $\phi$, the complex magnetic field is

$$
\boldsymbol{B}(\boldsymbol{r})=A_{0}\left[\sin \phi \partial_{\rho},-\cos \phi \partial_{\rho}, 0\right] \psi
$$

If we take $A_{0}$ real, and write the complex wavefunction $\psi(\rho, z)$ as $\psi_{r}+i \psi_{i}$, the real and imaginary parts of $\boldsymbol{B}(\boldsymbol{r})$ are both proportional to $\sin \phi,-\cos \phi, 0$, and are thus collinear. The magnetic field is therefore everywhere linearly polarized. The electric field is elliptically polarized, in general.

The dual of the TM beam under the transformation $\boldsymbol{E} \rightarrow \boldsymbol{B}, \boldsymbol{B} \rightarrow-\boldsymbol{E}$ (one of a set of duality transformations that leave the free space Maxwell equations unchanged) is the TE beam, transverse and linearly polarized in its electric field. The electric field lines are circles concentric with the beam axis (see figure 1 of [20], for example). However, both the TM and the TE beams disappear in the plane-wave limit: as $\psi \rightarrow e^{i k z}$ the electric and magnetic fields in both the TM and the TE beams tend to zero. Also, the fields are always zero on the beam axis; on both counts the exactly linearly polarized TE beam is not as interesting experimentally as the 'linearly polarized' beam about to be considered. A beam which does have a plane-wave limit is the linearly polarized 'LP' beam, with vector potential $\boldsymbol{A}=A_{0}[\psi, 0,0]$. The reason for the quotes around 'LP' is that the electric polarization is everywhere linear only in the plane wave limit. As we shall see, the deviation from linear polarization in finite beams is substantial, especially in the focal region. The magnetic and electric fields are

$$
\begin{aligned}
\boldsymbol{B} & =\nabla \times \boldsymbol{A}=A_{0}\left[0, \partial_{z},-\partial_{y}\right] \psi, \\
\boldsymbol{E} & =\frac{i}{k} \nabla(\nabla \cdot \boldsymbol{A})+i k \boldsymbol{A}=\frac{i A_{0}}{k}\left[\partial_{x}^{2}+k^{2}, \partial_{x} \partial_{y}, \partial_{x} \partial_{z}\right] .
\end{aligned}
$$

In the plane wave limit $\psi \rightarrow e^{i k z}, \boldsymbol{B} \rightarrow i k A_{0}[0,1,0] e^{i k z}, \boldsymbol{E} \rightarrow i k A_{0}[1,0,0] e^{i k z}$, which is the textbook linearly polarized plane wave with $\boldsymbol{E}$ and $\boldsymbol{B}$ transverse and mutually perpendicular. We again consider beams defined by wavefunctions $\psi$ independent of the azimuthal angle $\phi$, with the intention to use the 
wavefunctions $\psi_{0}, \psi_{b}$ and $\psi_{C}$ as examples. Then

$$
\begin{gathered}
\boldsymbol{B}=i k A_{0}\left[0, \partial_{z},-\sin \phi \partial_{\rho}\right] \psi \\
\boldsymbol{E}=\frac{i A_{0}}{k}\left[\cos ^{2} \phi \partial_{\rho}^{2}+\sin ^{2} \phi \rho^{-1} \partial_{\rho}+k^{2}, \sin \phi \cos \phi\left(\partial_{\rho}^{2}-\rho^{-1} \partial_{\rho}\right), \cos \phi \partial_{\rho} \partial_{z}\right] \psi
\end{gathered}
$$

Neither $\boldsymbol{E}$ nor $\boldsymbol{B}$ have real and imaginary parts collinear in general, but in the focal plane if $\partial_{\rho} \partial_{z} \psi=0$ the longitudinal component of will be zero and the transverse components will both be real, so only $\boldsymbol{E}_{r}$ is non-zero. Hence there can be circles of exact linear polarization in the focal plane. The electric field is also linearly polarized (along the $x$ direction) in the $x=0$ plane $(\cos \phi=0)$, as can be seen from (4.4.6). By symmetry the beam wavefunction is plane wave like (and thus has linear polarization) at the centre of the focal plane.

\subsection{Polarization properties of 'LP' beams based on $\psi_{0}, \psi_{b}$ and $\psi_{C}$}

We shall look at the consequences of the phase singularities for the polarization properties of an electromagnetic 'LP' beam built up from the scalar beam wavefunctions (4.1.3), (4.1.4) and (4.1.5). From 4.4.6) the electric polarization depends on the wavefunction and on its derivatives $\partial_{\rho} \psi, \partial_{\rho}^{2} \psi, \partial_{\rho} \partial_{z} \psi$. For the proto-beam $\psi_{0}$ all these derivatives are known analytically in the focal plane (equations 5.16 to 5.20 of [14]). Figure 4.5 shows the degree of linear polarization for an 'LP' beam derived from $\psi_{0}$. We see that there is perfect linear polarization at the centre of the focal plane, on the $x=0$ plane $\left(\cos \phi=0\right.$, so only $E_{x}$ is nonzero, from (4.4.6)), and also on circles where the spherical Bessel function $j_{2}(k \rho)$ is zero, since $\partial_{\rho} \partial_{z} \psi_{0}$ at $z=0$ is proportional to $i$ times $j_{2}(k \rho)$. The other derivatives entering into the expression (4.4.5) for the electric field are real in the focal plane, so when $j_{2}(k \rho)=0$ the electric vector is real, and $\Lambda=1$.

More surprising is the fact that perfect circular polarization exists, not far from the centre of the focal region. On the $x$ axis, when $\sin \phi=0, \cos \phi= \pm 1$, 
the electric field of (4.4.5) is proportional to $\left[\partial_{\rho}^{2}+k^{2}, 0, \pm \partial_{\rho} \partial_{z}\right] \psi$. For the protobeam we have from [14], equations (51.5), (5.17) and (5.19) that

$$
\begin{gathered}
\left(\partial_{\rho}^{2}+k^{2}\right) \psi_{0}=6(k \rho)^{-3}\left(2 J_{1}(k \rho)-k \rho J_{0}(k \rho)\right), \\
\partial_{\rho} \partial_{z} \psi_{0}=-2 i(k \rho)^{-1} j_{2}(k \rho) .
\end{gathered}
$$

Hence when the expressions 4.5.1) and 4.5.2) are equal in magnitude the electric field will be proportional to $[1,0, \pm i]$, corresponding to perfect circular polarization in the $x z$ plane. The $C$ lines intersect the focal plane at $k|x| \approx 3.98$, $5.44,6.94,8.79 \ldots$ The points nearest to the focal centre, at $k x \approx \pm 3.98, y=$ $0, z=0$, have electric field magnitude equal to $18.6 \%$ of the field at the centre, a significant fraction.

Figure 4.6 shows how the polarization of the 'LP' Carter beam varies with position, when the tightness of focus parameter $k b=5$. Note that there is a very strong deviation from linear polarization between the inner two zeros of $\psi_{C}$, which are about to coalesce (event $c$, at $k b \approx 5.255$, as discussed in Section 4.3. The beam is linearly polarized at the centre of the focal region, by design, and there are also circles of linear polarization in the focal plane at distances from the beam axis where $\partial_{\rho} \partial_{z} \psi=0$. An animation of the changes that occur as increases through the transition values discussed in Section 4.3 is available online. Figure 4.7 shows the polarization of a 'linearly polarized' beam based on the wavefunction $\psi_{b}$ defined in 4.1.4.

\subsection{Discussion}

We have compared two families of beams $\psi_{C}$ and $\psi_{b}$, and their common tight focusing limit $\psi_{0}$. The beam wavefunction given in 4.1.3 was proposed in 1973 by Carter [15] and the topology of its phase singularities was analysed by Berry [16]. This cylindrically symmetric wavefunction is parametrized by $k b$, where $k=\omega / c$ is the wavenumber, and $b$ is a length. The Carter wavefunction isophase surfaces meet on the wavefunction zeros which, for values of the dimensionless parameter $k b$ smaller than the critical value 4.91961, all lie on 
circles in the focal plane. However, at larger $k b$ values the interplay of the evolution of the surfaces on which $\operatorname{Re}\left(\psi_{C}\right)=0$ and $\operatorname{Im}\left(\psi_{C}\right)=0$ and of their intersections (on the complex zeros of $\psi_{C}$ ) produces zeros which lie off the focal plane. This behaviour was shown numerically by Carter, and classified by Berry. The wavefunction $\psi_{b}$ defined in (4.1.4) forms a family also characterized by the parameter which determines the tightness of focus.

The wavefunctions $\psi_{C}$ and $\psi_{b}$ coalesce into the proto-beam $\psi_{0}$ as $k b \rightarrow 0$, but become topologically different at intermediate and large values of the parameter $k b$. It is interesting that the loosening of the focus leads to the differentiation. To see the reason for the differences we shall compare the weight functions of $\psi_{C}$ and $\psi_{b}$. In terms of the dimensionless variables we can write the beam wavefunctions (normalized to unity at the origin) as

$$
\begin{gathered}
\psi_{C}=\frac{\beta e^{\beta / 2}}{e^{\beta}-1} \int_{0}^{1} d \eta \eta e^{\frac{b}{2} \eta^{2}+i \xi \eta} J_{0}\left(\alpha \sqrt{1-\eta^{2}}\right) \\
=\int_{0}^{1} d \eta W_{C}(\beta, \eta) e^{i \xi \eta} J_{0}\left(\alpha \sqrt{1-\eta^{2}}\right), \\
\psi_{b}=\frac{\beta^{2}}{e^{\beta}(\beta-1)+1} \int_{0}^{1} d \eta \eta e^{\eta(\beta+i \xi)} J_{0}\left(\alpha \sqrt{1-\eta^{2}}\right) \\
=\int_{0}^{1} d \eta W_{b}(\beta, \eta) e^{i \xi \eta} J_{0}\left(\alpha \sqrt{1-\eta^{2}}\right) .
\end{gathered}
$$

The weight functions of these generalized Bessel beams are

$$
\begin{aligned}
& W_{C}(\beta, \eta)=\frac{\beta^{2} e^{\beta / 2}}{e^{\beta}-1} \eta e^{\beta \eta^{2} / 2}, \\
& W_{b}(\beta, \eta)=\frac{\beta^{2}}{e^{\beta}(b-1)+1} \eta e^{\beta \eta} .
\end{aligned}
$$

The weight function $W_{C}$ is always larger at small $\eta$ (small longitudinal wave vector component $q$ ), exponentially so at large $\beta=k b . W_{b}$ is always larger as $\eta \rightarrow 1(q \rightarrow k)$. The weight functions are equal at

$$
\eta_{c}=\left(\frac{q}{k}\right)_{c}=1-\sqrt{1+\frac{2}{\beta} \ln \left(\frac{\beta\left(e^{\beta / 2}-1\right)}{e^{\beta}(\beta-1)}\right)} .
$$


This cross over value of $q / k$ is always greater than $1-6^{-1 / 2} \approx 0.59$ (its limiting value at $\beta \rightarrow 0$ ), and tends to unity for large $\beta$ as $1-\sqrt{2} \beta^{-1}$. Thus the Carter beam emphasises the transverse component $\kappa$ of the wavevector, while $\psi_{b}$ emphasises the longitudinal wavevector component $q$. The function $\psi_{b}$ is the more 'paraxial' beam wavefunction at large $k b$, as is seen in the comparison of exact and paraxial beam wavefunctions in [22].

Section 4.5 explored the polarization properties of 'LP' beams based on these three wavefunctions. Of importance in experiments where the polarization of an electromagnetic beam is involved, is the very strong effect that the wavefunction zeros (on and off the focal plane) have on the polarization of the light beam. We showed examples of beams linearly polarized in the plane wave limit, yet with regions of circular polarization surrounding the central focal region, located near the wavefunction zeros. In the most tightly focused case, the perfect circular polarization points nearest to the focal centre have electric field magnitude equal to a significant fraction of the field at the (linearly polarized) centre of the beam.

Berry [48] considered the connection between optical vortices and angular momentum, and between circular polarization singularities and angular momentum, concluding that there is usually no connection. Here we saw that the zeros of the beam wavefunction are strongly correlated with polarization anomalies. 


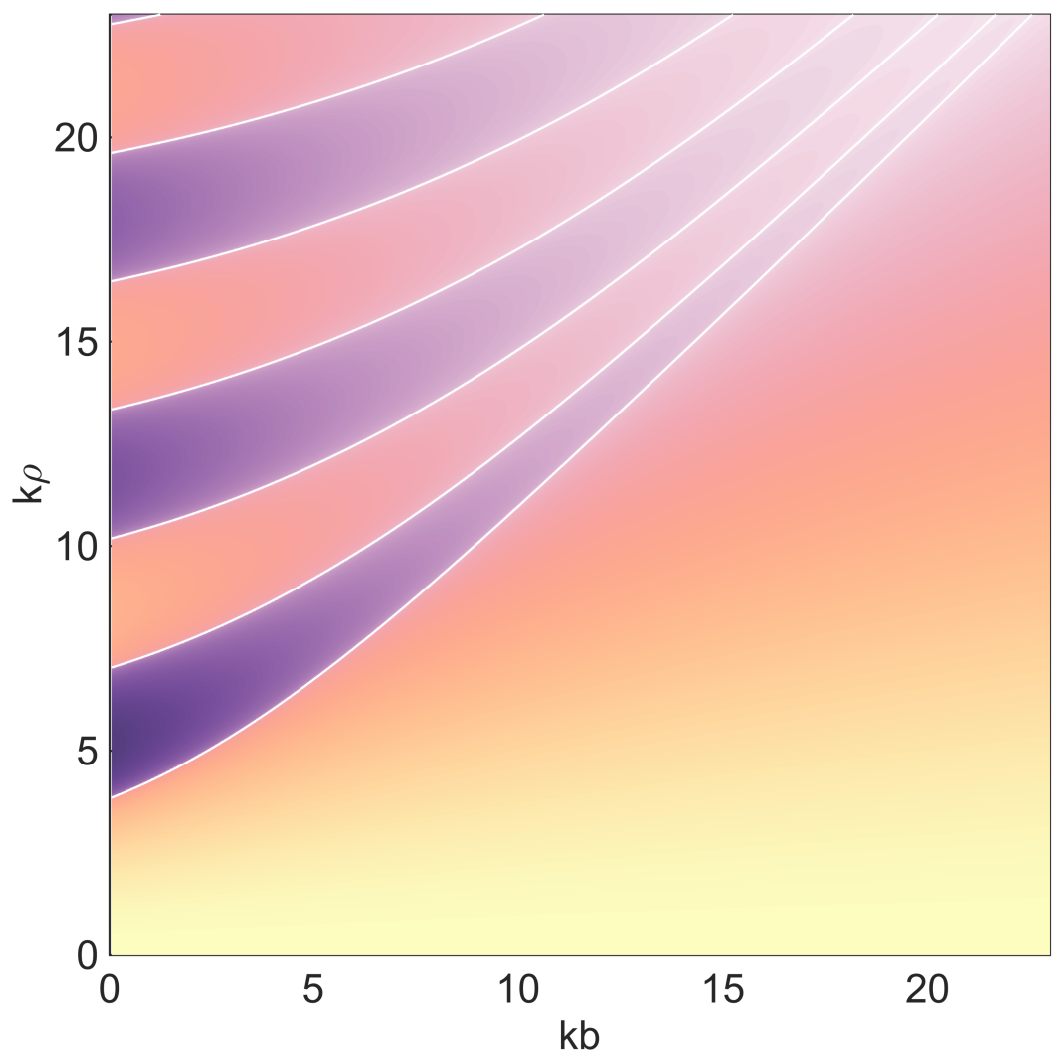

Figure 4.2: Zeros of $\psi_{b}(\rho, 0)$ as a function of $k b$ (white curves). Also shown are the wavefunction focal plane values (negative: dark shading; zero: white; positive: light shading). As $k b \rightarrow 0$ the wavefunction zeros tend to those of the proto-beam. These are the zeros of $J_{1}(k \rho) / k \rho$, at $k \rho \approx 3.83,7.02,10.17,13.32, \ldots$. 

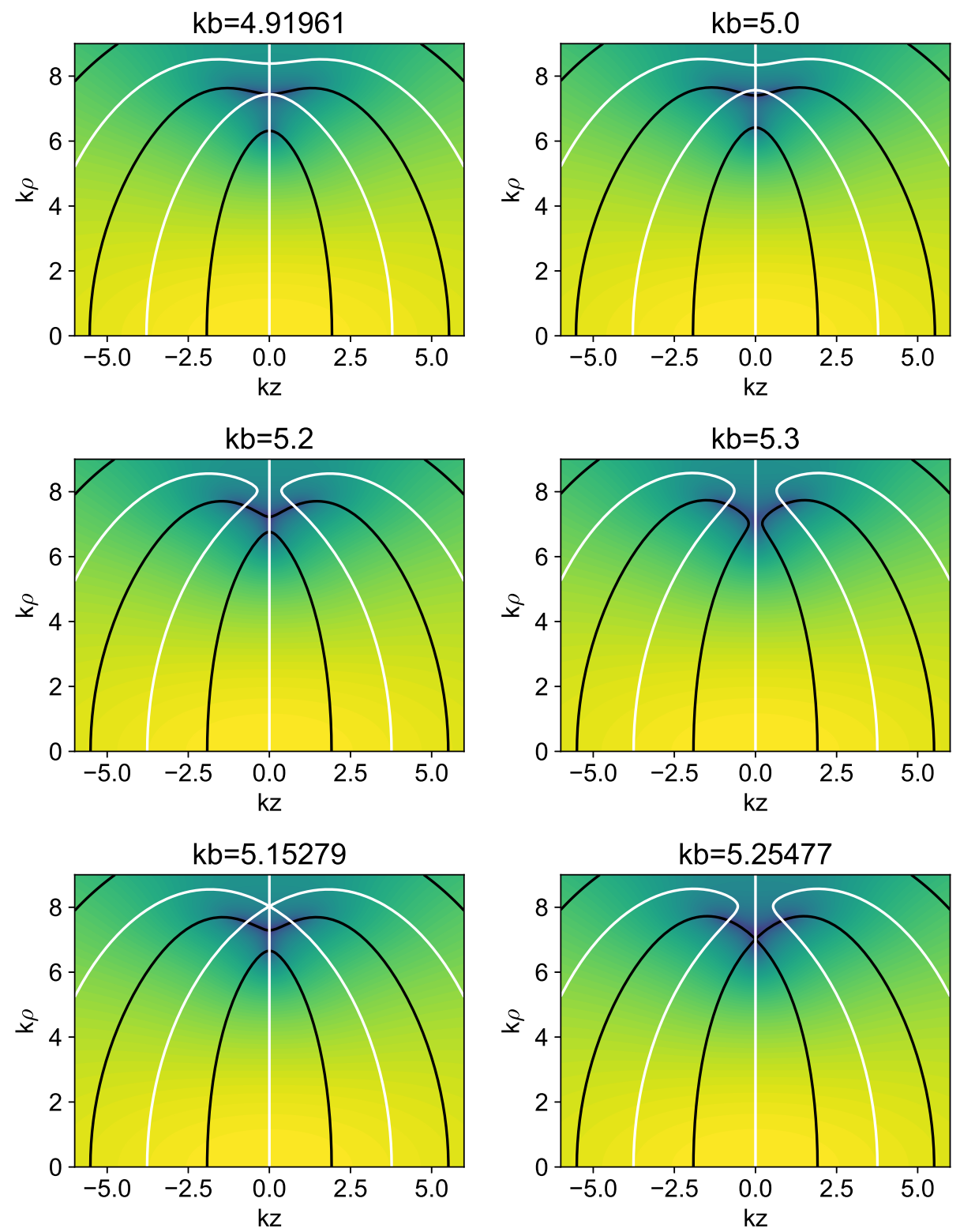

Figure 4.3: Surfaces of $\operatorname{Re}\left(\psi_{C}\right)$ (black) and $\operatorname{Im}\left(\psi_{C}\right)$ (white) in the focal region, for six values of $k b$. Included are three transition values $k b=$ 4.91961, 5.15279, 5.25477, discussed in the text. The three-dimensional picture is obtained by rotating the figures about the beam (horizontal) axis. The shading is in proportion to the logarithm of $\psi_{C}$. 


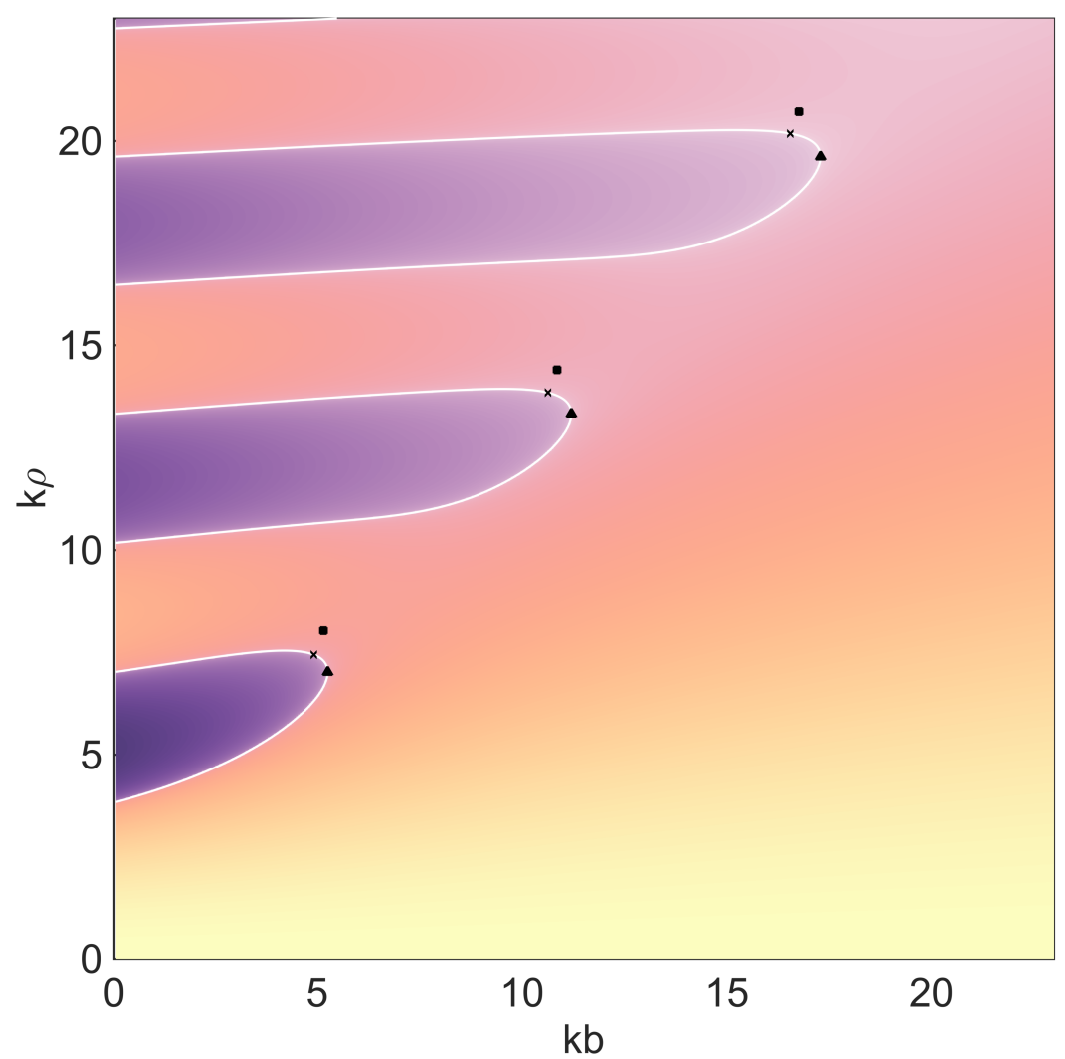

Figure 4.4: Variation with $k b$ of the focal plane zeros of the Carter wavefunction (4.1.3) (white curves). Also shown are the wavefunction focal plane values (shading, as in figure 4.2. At $k b=0$ the wavefunction zeros are again those of $J_{1}(k \rho) / k \rho$, as in figure 4.2 . The transition points $a, b, c$ discussed in the text are indicated by crosses, squares and triangles, respectively. 


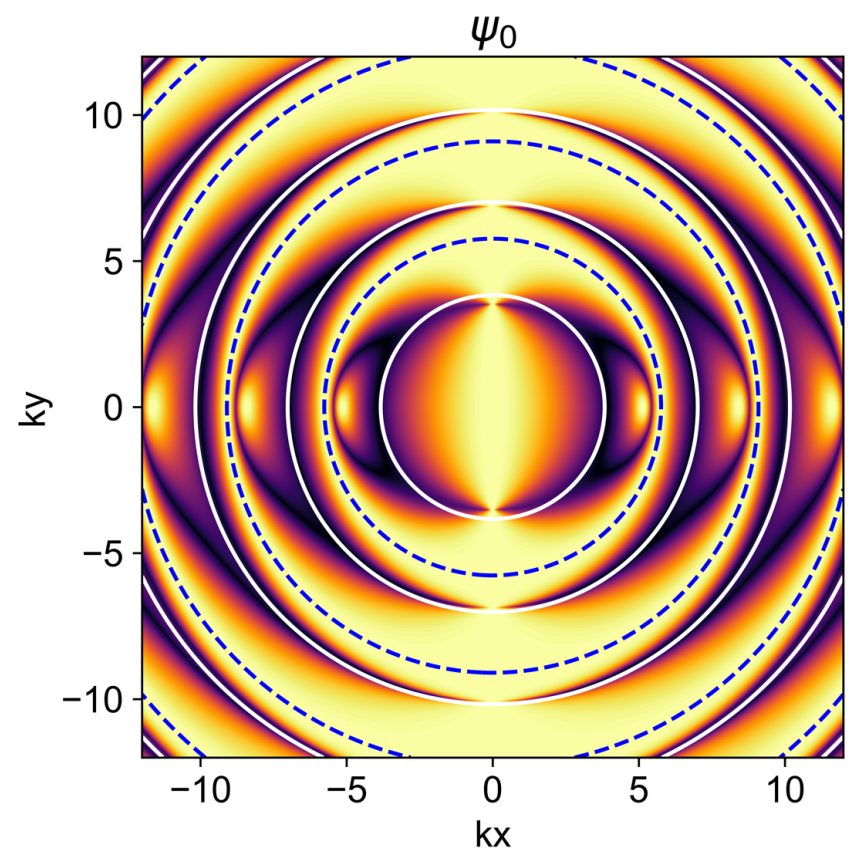

Figure 4.5: Degree of electric field linear polarization $\Lambda$ for a 'linearly polarized' $\psi_{0}$ beam, in the focal plane. Light shading denotes linear and dark shading circular polarizations. The white circles are the zeros of $\psi_{0}$, namely those of $J_{1}(k \rho) / k \rho$. The dashed circles are loci of perfect linear polarization, $\Lambda=1$, as discussed in the text. On the horizontal axis $(y=0)$ there are points of perfect circular polarization, also discussed in the text. 


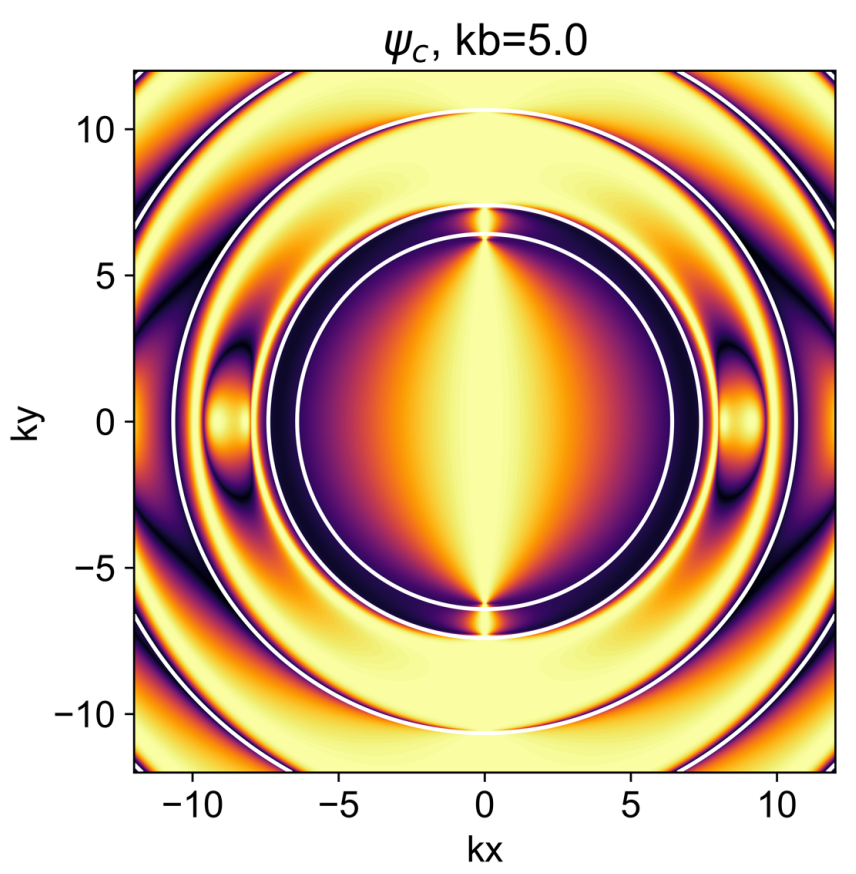

Figure 4.6: The measure $\Lambda$ of linear polarization of the electric field of an 'LP' beam based on $\psi_{C}$, shown in the focal plane for $k b=5$. Light colour denotes linear and dark colour circular polarizations. There are circles of perfect linear polarisation when $\partial_{\rho} \partial_{z} \psi=0$, at $k \rho \approx 7.9,9.9 \ldots$ Note the correlation of the deviation from linear polarization with the circles of zero $\psi$ (white), especially between the innermost pair of zeros which occur at $k \rho \approx 6.4$ and 7.4 when $k b=5$. An animation for variable $k b$ is available in the supplementary material online (http://stacks.iop.org/JOPT/19/105609/mmedia). 


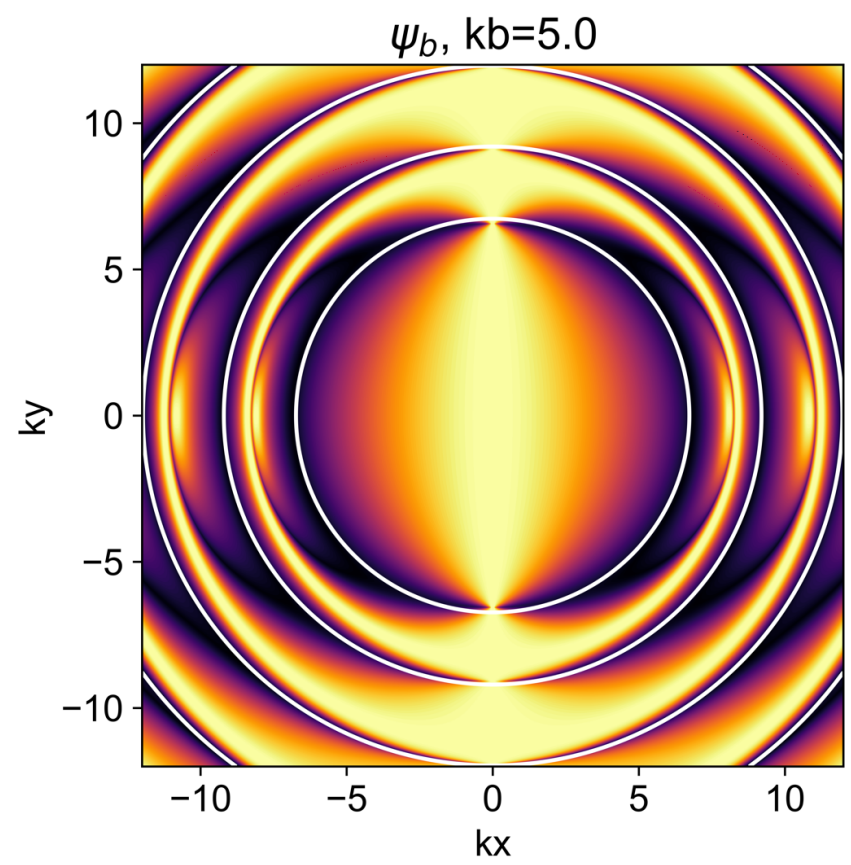

Figure 4.7: The measure $\Lambda$ of linear polarization of the electric field of an 'LP' beam based on $\psi_{b}$, shown in the focal plane for $k b=5$. Light colour denotes linear and dark colour circular polarizations. There are circles of perfect linear polarization when $\partial_{\rho} \partial_{z} \psi=0$, at $k \rho \approx 8.4,11.2 \ldots$. Note the correlation of the deviation from linear polarization with the circles of zero $\psi$ (white), the first three of which occur at $k \rho=6.79 .2$ and 12.0 when $k b=5$. 


\section{Chapter 5}

\section{Reflection of focused beams off a delta potential}

\subsection{Introduction}

One of the exactly solvable potentials for Schrödingers equation is the delta potential well in 1D. This can also be used in 3D to represent systems such as planar boundaries between media, oxide layers, etc. In cylindrical coordinates, such a potential gives (for monochromatic waves)

$$
-\frac{\hbar^{2}}{2 m} \nabla^{2} \psi+\frac{\hbar^{2} Q}{m} \delta(z) \psi=\hbar \omega \psi
$$

where $2 Q$ is the barrier strength. Solving this for a plane wave in $z$ is a textbook problem (See Griffiths [1. Chapter 1, Section 4] or Theory of Reflection [8. Chapter 2]), and the solution will apply to any eigenstate of $\hat{k}_{z}$. We shall restrict ourselves to azimuthally symmetric systems, so the scattering states of interest are Bessel beams, propagating normal to the plane. For an incident Bessel beam,

$$
\psi_{0}=e^{i q z} J_{0}(\kappa \rho), \quad q^{2}+\kappa^{2}=k^{2}=\frac{2 m \omega}{\hbar},
$$


the scattering state is

$$
\psi(\rho, \phi, z)=\left\{\begin{array}{l}
\left(e^{i q z}-\frac{i Q}{q+i Q} e^{-i q z}\right) J_{0}(\kappa \rho), \quad z<0, \\
\frac{q}{q+i z} e^{i q z} J_{0}(\kappa \rho), \quad z>0 .
\end{array}\right.
$$

In general, a beam will not have a normalisable probability density over all space, so the normalisation condition we shall use is that the incident probability flux is unity,

$$
\int \mathrm{d}^{2} r \boldsymbol{j}_{0}\left(\rho, \phi, z_{0}\right) \cdot \hat{z}=1,
$$

where $j_{0}$ is the incident probability current.

A Bessel beam is not normalisable in this way, as the radial integral is infinite, however for suitable superpositions of Bessel beams the integral is finite, and thus the state is normalisable. Thus, given an incident beam

$$
\psi_{0}(\rho, z)=\int_{0}^{k} \mathrm{~d} q f(k, q) e^{i q z} J_{0}(\kappa \rho)
$$

the solution will be

$$
\psi(\rho, z)=\left\{\begin{array}{l}
\int_{0}^{k} \mathrm{~d} q f(k, q) J_{0}(\kappa \rho)\left(e^{i q z}-\frac{i Q}{q+i Q} e^{-i q z}\right), \quad z<0 \\
\int_{0}^{k} \mathrm{~d} q f(k, q) J_{0}(\kappa \rho) \frac{q}{q+i Q} e^{i q z}, \quad z>0 .
\end{array}\right.
$$

The normalisation condition is

$$
2 \pi \int_{0}^{k} \mathrm{~d} q|f(k, q)|^{2}=1
$$

As the incident current is normalised to unity, the outgoing current is given by

$$
2 \pi \int_{0}^{k} \mathrm{~d} q|f(k, q)|^{2} \frac{q^{2}}{q^{2}+Q^{2}}
$$

Translating the focal plane by $a$ will multiply the weight function by an $e^{i q a}$ factor, and thus will not modify the transmitted current. 


\subsection{Linear weight function}

A particularly simple beam is referred to as the 'proto-beam' by Lekner, who has extensively studied its properties [14]. A plot of the free space beam is given in Figure 5.1. Its normalised weight function is given by $f_{P}(q / k)=\sqrt{\frac{3}{2 \pi k^{3}}} q$, and represents a beam very tightly focused at the origin.

The focal plane wave-function is given by a Jinc function,

$$
\psi_{P}(\rho, z=0)=\sqrt{\frac{3}{2 \pi k^{3}}} \frac{J_{1}(k \rho)}{k \rho} .
$$

and has an infinite number of wave-function zeroes in the focal plane. Around these zeroes, the probability current circulates. We expect that when the zeros are situated on or near the barrier, they will strongly affect the probability current near the barrier.

The transmission probability (transmittance) is defined as the ratio of transmitted current over incident,

$$
T=\frac{\int \mathrm{d}^{2} r \boldsymbol{j}_{T} \cdot \hat{z}}{\int \mathrm{d}^{2} r \boldsymbol{j}_{0} \cdot \hat{z}}
$$

which reduces to $\int \mathrm{d}^{2} r \boldsymbol{j}_{T} \cdot \hat{z}$ if the incident current is normalised to unity.

For the proto-beam, the transmittance is given by

$$
T_{P}(Q)=2 \pi \int_{0}^{k} \mathrm{~d} q|f(k, q)|^{2} \frac{q^{2}}{q^{2}+Q^{2}}=1-3 Q^{2} / k^{2}+3 Q^{3} / k^{3} \arctan (k / Q) .
$$

Contrast this with the (normally incident) Bessel beam transmittance of

$$
T_{q}(Q)=\frac{q^{2}}{q^{2}+Q^{2}} .
$$

The proto-beam's transmittance is very close to that of a Bessel beam with $z$ momentum $q=\sqrt{3 / 5} k$ (Figure 5.2 , the value of which can be obtained by comparing asymptotic series.

$$
\begin{gathered}
T_{P}(Q / k) \approx \frac{3}{5} k^{2} Q^{-2}+\mathscr{O}\left(k^{4} Q^{-4}\right), \\
T_{q}(Q / k) \approx q^{2} Q^{-2}+\mathscr{O}\left(k^{4} Q^{-4}\right) .
\end{gathered}
$$




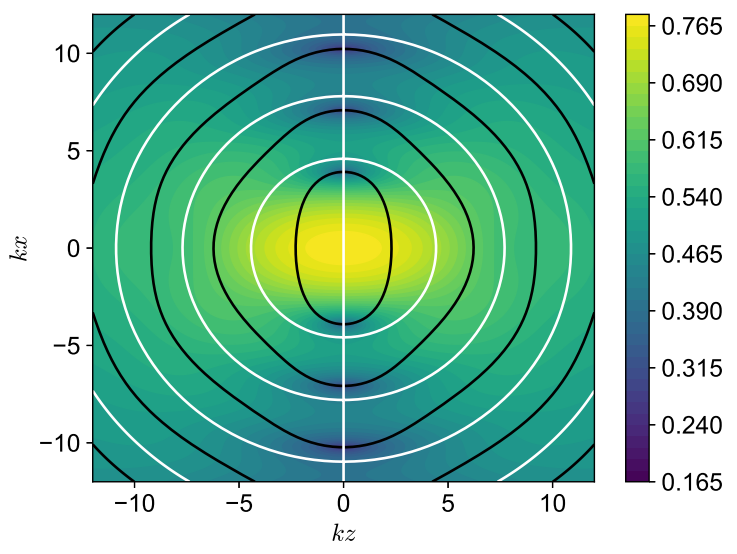

Figure 5.1: Proto beam free space modulus (shading), and real (black) and imaginary (white) zero contours. Real and imaginary zero contours intersect at points of zero modulus, with probability current circulating around these points. Modulus has been scaled to the power of 0.2. All zeros lie in the focal plane $k z=0$. Note the imaginary zero in the entire focal plane. This will always be true for real weight functions, as they are purely real in the focal plane. This does not imply all their zeros must be in the focal plane; see for example the Carter beam in Chapter 4 .

Figure 5.3 shows the modulus of the proto-beam with the focal plane incident on the barrier, for $Q=0.01 k$ and $Q=k$. The zeros are shifted off plane, and the real and imaginary zero contours on the incident side are shifted closer together. In the infinite barrier limit, the transmittance is zero, and the reflected and transmitted wavefunctions are perfectly in phase. The wavefunction approaches

$$
\psi_{L} \rightarrow 2 i \operatorname{Im}\left(\psi_{0}\right)
$$

The trough region seen in Figure 5.3(b) approach the zeros of the imaginary part of the incident wave function, and for an infinite barrier are coincident.

Shifting the focal plane can have a dramatic effect on the zeros. Figures 5.4 and 5.5 show the effect of shifting the focal plane by $k a$ between \pm 4 relative to 


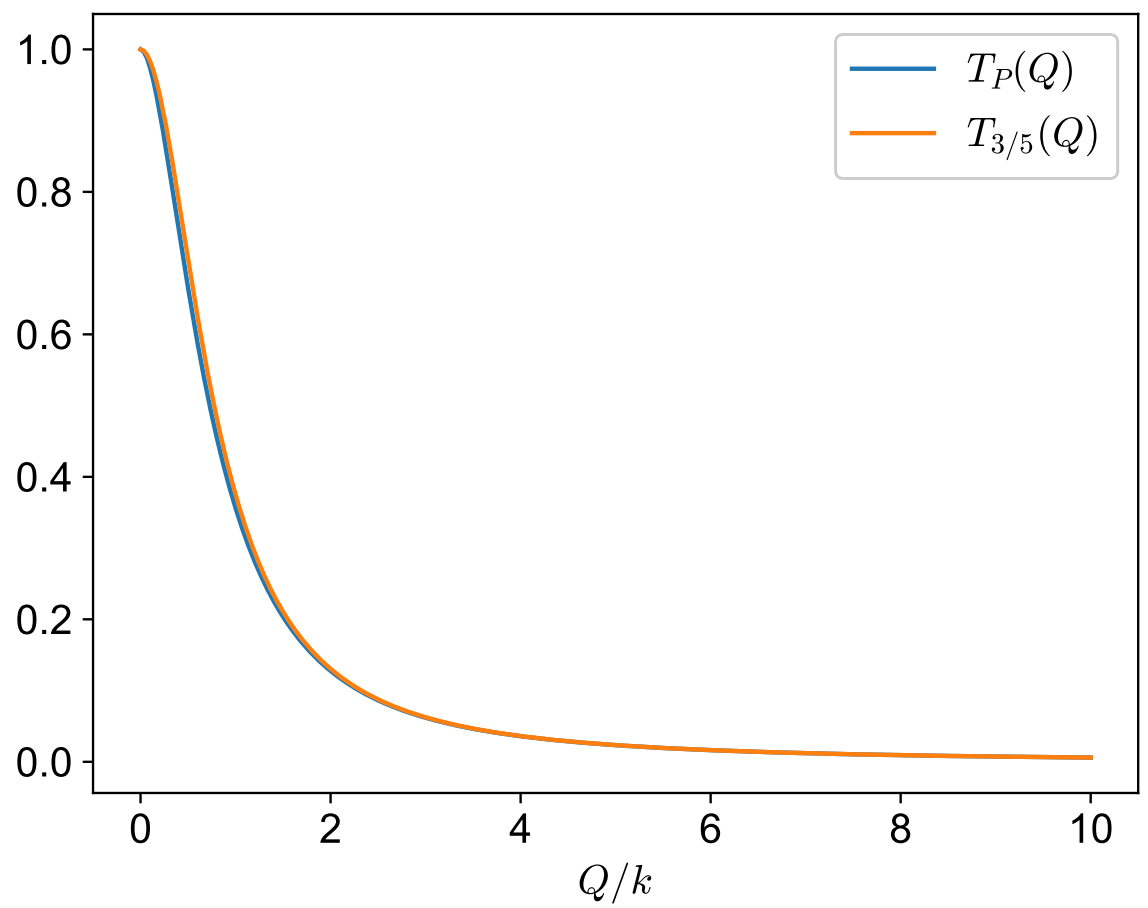

Figure 5.2: Comparison of proto-beam transmittance to that of a Bessel beam (or plane wave) with momentum $k_{z}=\sqrt{3 / 5} k$.

the barrier. For negative shifts (placing the focus in the transmitted side), the incident functions zeros remain on the transmitted side. However, there can also be topological changes in the phase surfaces, that create new zeros on the incident side. Compare this to the Carter wavefunction of Chapter 4 , which has topological changes for critical values of the focal parameter $k b$.

\subsection{Bessel beam incident at an angle}

So far we have considered normal incidence, so that the overall system has a cylindrical symmetry. We shall now consider the reflection of a Bessel beam incident at an angle $\theta$ to the normal of the potential barrier. First, we shall write 


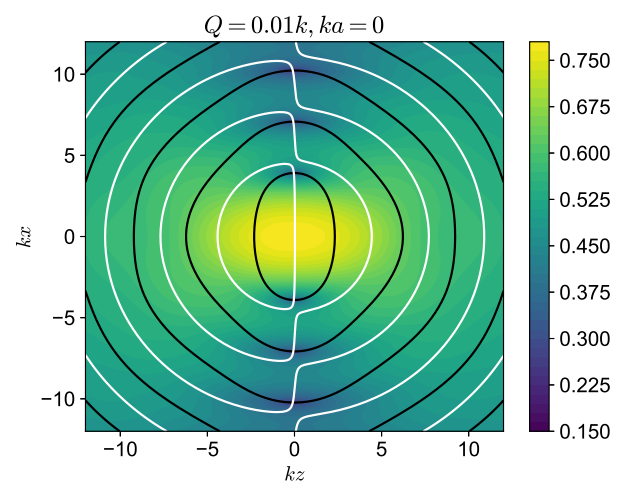

(a)

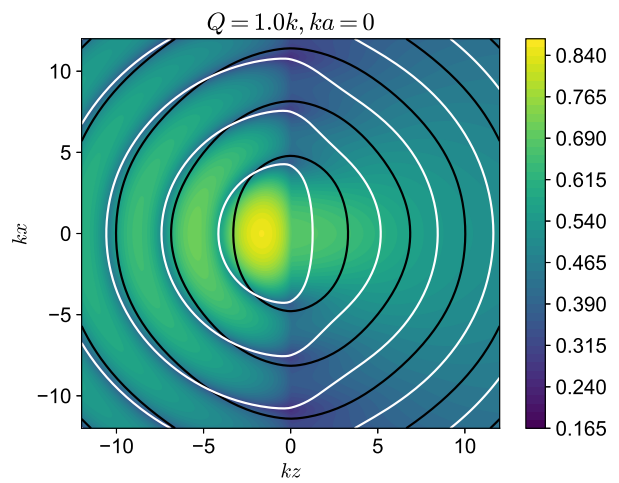

(b)

Figure 5.3: Modulus and zero contours for a normally incident proto beam focused on a potential barrier with strength $Q=0.01 k$, Figure 5.3(a) and $Q=k$, Figure 5.3(b), For weak barriers, the zeros are shifted only slightly, however the topology of the imaginary zero contours is drastically changed compared to free beam, Figure 5.1. As barrier strength is increased, the zeros rapidly shift off the focal plane, and the real contours approach the imaginary contours on the reflected side. In the infinite barrier limit, the real and imaginary zero contours merge, coincident with the imaginary zeros of the free beam.

the Bessel beam as the conical superposition of plane waves with a conical angle $\alpha$ with the $z^{\prime}$ axis, the beams direction of propagation.

$$
\psi\left(x^{\prime}, y, z^{\prime}\right)=\frac{1}{2 \pi} \int_{0}^{2 \pi} \mathrm{d} \phi \exp \left(i k\left[z^{\prime} \cos \alpha+x^{\prime} \sin \alpha \cos \phi+y \sin \alpha \sin \phi\right]\right) .
$$

We shall consider for now $\alpha<\pi / 2-\theta$, so that all plane-wave components of the beam are incident to the barrier. The generalisation for arbitrary beams will be given in the following section.

Labelling the plane of incidence as the $x, z$ plane, with $z$ the barrier normal coordinate, the beam normal coordinates $x^{\prime}, y, z^{\prime}$ (note that $y$ is unmodified) are obtained from the barrier normal coordinates $x, y, z$ via the rotation

$$
\left(\begin{array}{l}
z^{\prime} \\
x^{\prime}
\end{array}\right)=\left(\begin{array}{cc}
\cos \theta & -\sin \theta \\
\sin \theta & \cos \theta
\end{array}\right)\left(\begin{array}{l}
z \\
x
\end{array}\right) .
$$




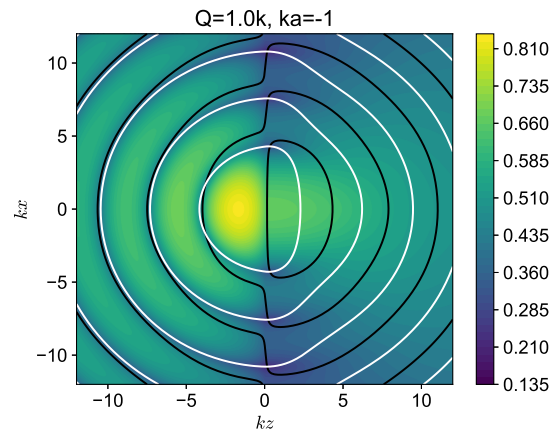

(a)

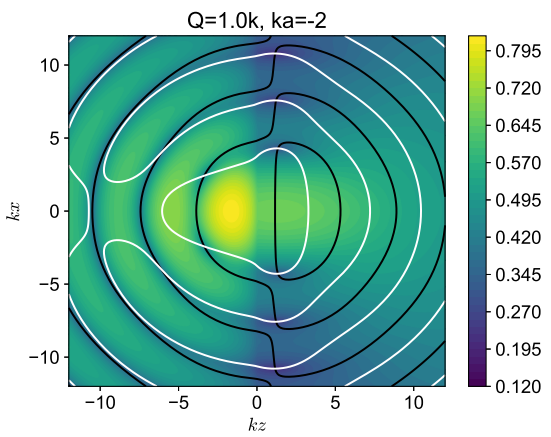

(b)

Figure 5.4: Comparison of proto-beam translated by $k a=-1,-2$, for barrier strength $Q=k$. Note that the zeros of the proto-beam are shifted into the transmitted region, as $k a<0$ shifts the focal plane into the transmitted side. Also note the topological change of the phase surfaces in the incident region, that results in the creation of new zeros. This is reminiscent of the Carter wavefunction for Chapter 4

It will be convenient to define

$$
\begin{aligned}
q(\phi) & =k(\cos \alpha \cos \theta+\sin \alpha \sin \theta \cos \phi), \\
\kappa_{x}(\phi) & =k(\sin \alpha \cos \theta \cos \phi-\cos \alpha \sin \theta), \\
\kappa_{y}(\phi) & =\sin \alpha \sin \phi \\
\boldsymbol{\kappa} & =\kappa_{x} \hat{x}+\kappa_{y} \hat{y} \\
\boldsymbol{\rho} & =x \hat{x}+y \hat{y}
\end{aligned}
$$

We can see that in the barrier normal coordinates the wavefunction is given by

$$
\psi(x, y, z)=\frac{1}{2 \pi} \int_{0}^{2 \pi} \mathrm{d} \phi \exp (i \boldsymbol{\kappa}(\phi) \cdot \boldsymbol{r}) .
$$

The wavefunction on either side of the barrier will be

$$
\begin{gathered}
\psi_{L}(x, y, z)=\frac{1}{2 \pi} \int_{0}^{2 \pi} \mathrm{d} \phi e^{i \boldsymbol{\kappa}(\phi) \cdot \boldsymbol{\rho}}\left(e^{i q(\phi) z}-A_{R}(\phi) e^{-i q(\phi) z}\right), \\
\psi_{R}(x, y, z)=\frac{1}{2 \pi} \int_{0}^{2 \pi} \mathrm{d} \phi e^{i \boldsymbol{\kappa}(\phi) \cdot \boldsymbol{\rho}} A_{T}(\phi) e^{i q(\phi) z} .
\end{gathered}
$$


with the transmission and reflection amplitudes given by

$$
\begin{aligned}
& A_{T}(\phi)=\frac{q(\phi)}{q(\phi)+i Q}, \\
& A_{R}(\phi)=\frac{i Q}{q(\phi)+i Q} .
\end{aligned}
$$

The integrals are not tractable symbolically, however they are straightforward to compute numerically. The transmitted beam will still have the same direction of propagation, and is still an eigenfunction of $p_{z}^{\prime}$, with the same eigenvalue. This can be seen by transforming back to the primed coordinates

$$
\psi_{R}\left(x^{\prime}, y, z^{\prime}\right)=\frac{e^{i k \cos \alpha z^{\prime}}}{2 \pi} \int_{0}^{2 \pi} \mathrm{d} \phi e^{i k \sin \alpha\left[x^{\prime} \cos \phi+y \sin \phi\right]} A_{T}(\phi) .
$$

Similarly, the reflected beam will have the angle of reflection equal to the angle of incidence. However, the phase behaviour of the resulting wavefunction is considerably more complex than before, and there are strong diffraction effects on the reflected side, Figure 5.6 .

The explicit expression for the transmitted wavefunction radial to the propagation direction is

$$
\frac{1}{2 \pi} \int_{0}^{2 \pi} \mathrm{d} \phi e^{i k \sin \alpha\left[x^{\prime} \cos \phi+y \sin \phi\right]} \frac{\cos \alpha \cos \theta+\sin \alpha \sin \theta \cos \phi}{\cos \alpha \cos \theta+\sin \alpha \sin \theta \cos \phi+i Q / k} .
$$

A Bessel beam has an even weighting to all momenta with projection $k \sin \alpha$. In addition, the incident beam has an entirely real radial profile. In contrast, the transmitted beam has a complex radial profile, with an asymmetric angular weight function. The components with negative $k_{x}$, in the direction of the interface, are reduced. Figure 5.7 gives the polar plots of the real and imaginary parts of the transmission amplitude $A_{T}(\phi)$.

The transmitted beam can be expanded as a series in higher order Bessel beams. First, we note that in cylindrical coordinates we have (see Section)

$$
e^{i \boldsymbol{\kappa} \cdot \boldsymbol{\rho}}=e^{i \kappa \rho \cos \left(\phi-\phi^{\prime}\right)},
$$

with $\phi$ the angle of $\rho$, and $\phi^{\prime}$ the angle of $\kappa$. Next, we use the identity

$$
e^{i \kappa \rho \cos \left(\phi-\phi^{\prime}\right)}=\sum_{m=-\infty}^{\infty} i^{m} J_{m}(\kappa \rho) e^{i m\left(\phi-\phi^{\prime}\right)} .
$$


Thus, the series expansion is given by

$$
\begin{gathered}
\psi_{R, \perp}(\rho, \phi)=\sum_{m=-\infty}^{\infty} C_{m} J_{m}(k \sin \alpha \rho) e^{i m \phi}, \\
C_{m}=\frac{i^{m}}{2 \pi} \int_{0}^{2 \pi} \mathrm{d} \phi^{\prime} e^{-i m \phi^{\prime}} T\left(\phi^{\prime}\right) .
\end{gathered}
$$

The expansion coefficients are simply given by the Fourier series of the transmission function, up to a phase. These can be explicitly calculated using contour integration. Writing $C_{m}=i^{m} B_{m}$, we have two cases to consider, the first when $m>0$, the second when $m<0$. For either case, make the substitution $\zeta=\exp (\operatorname{sign}(m) i \phi)$. When $m<0$ this gives a clockwise contour about the unit circle, for $m>0$ this gives anticlockwise. The integrand will be

$$
\begin{aligned}
B_{m} & =\frac{\operatorname{sign}(m)}{2 \pi i} \oint d \zeta \frac{\zeta^{|m|-1} F(\zeta)}{G(\zeta)} \\
F(\zeta) & =b+2 a \zeta+b \zeta^{2} \\
G(\zeta) & =b+2(a+i Q) \zeta+b \zeta^{2} .
\end{aligned}
$$

We can factorise $G$ by its roots, giving

$$
G(\zeta)=b\left(\zeta-\zeta_{+}\right)\left(\zeta-\zeta_{-}\right), \quad \zeta_{ \pm}=b^{-1}\left(-(a+i Q) \pm \sqrt{(a+i Q)^{2}+b^{2}}\right) .
$$

If the roots lie within the unit circle, the contour integral will be equal to $2 \pi i$ times the sum of the residues at the roots, times -1 for the clockwise winding when $m<0$. This gives the same result for either case,

$$
B_{m}=B_{-m}=b^{-1}\left(\zeta_{+}-\zeta_{-}\right)^{-1}\left(S\left(\zeta_{+}\right) \zeta_{+}^{|m|-1} F\left(\zeta_{+}\right)-S\left(\zeta_{-}\right) \zeta_{-}^{|m|-1} F\left(\zeta_{-}\right)\right),
$$

where $S$ is the step function such that

$$
S(\zeta)= \begin{cases}1, & |S|<1 \\ 0, & \text { otherwise }\end{cases}
$$

The $m=0$ coefficient is given by

$$
B_{0}=\frac{1}{2 \pi i} \oint \mathrm{d} \zeta \frac{F(\zeta)}{\zeta G(\zeta)}=1+b^{-1}\left(\zeta_{+}-\zeta_{-}\right)^{-1}\left(\zeta_{+}^{-1} S\left(\zeta_{+}\right) F\left(\zeta_{+}\right)-\zeta_{-}^{-1} S\left(\zeta_{-}\right) F\left(\zeta_{-}\right)\right)
$$


The validity of these expressions was confirmed by comparing the results with numeric integration for a range of different parameters. Since Bessel beams are also the eigenfunctions of $L_{z}$, the probability of measuring the beam to have angular momentum per unit thickness $\hbar m$ is given by $\left|C_{m}\right|^{2}$. Note that the beam is not radially normalisable, so the expectation value angular momentum cannot be computed analytically via direct integration over the radial plane. However, if the Fourier coefficients are calculated, we can normalise based off the angular momentum probabilities,

$$
\sum_{m=-\infty}^{\infty}\left|C_{m}\right|^{2}=1
$$

the sum being guaranteed to converge due to the square integrability of $A_{T}(\phi)$, and the expectation value can be obtained via

$$
\left\langle L_{z}\right\rangle=\hbar \sum_{m=-\infty}^{\infty} m\left|C_{m}\right|^{2} .
$$

This is equivalent to an integral over the transmission function.

$$
\left\langle L_{z}\right\rangle=\frac{\hbar}{2 \pi i} \int_{0}^{2 \pi} \mathrm{d} \phi A_{T}^{*}(\phi) \frac{\partial A_{T}(\phi)}{\partial \phi} .
$$

There are various ways to show that the expectation value will be zero. Since $A_{T}(\phi)$ can be written as a function of $\cos \phi, T(\phi)=f(\cos \phi)$, the derivative can be written as $-\sin \phi f^{\prime}(\cos \phi)$. Thus, since the transmission function is even, and the derivative will be odd, the integral will be zero. This can also be seen by the fact that $\left|C_{m}\right|=\left|C_{-m}\right|$, or that the system has a symmetry under $y \rightarrow-y$.

We shall now compare the radial intensity profile to that of the incident Bessel beam. The $J_{0}$ beam has rings of real zeros in the radial profile, which when extended in the propagation direction give cylindrical sheet zero surfaces. The transmitted beam no longer has ring zeros, as the radial function is no longer purely real, and the real and imaginary zeros do not coincide, Figure 5.8. The real and imaginary zero contours are ring shaped, and offset slightly, so that each pair crosses at two points. Extending these points in the propagation direction give isolated zero lines, around which there is an associated circulation (Figure 5.9) of probability current. As such, the probability current 
streamlines are no longer straight in the propagation direction, but are instead helical around the zero lines, Figure 5.9. Each pair of crossings has an opposite topological charge, so that the helicity is opposite, and the net angular momentum is zero.

\subsection{General beam-forms}

A general beam is given by the Fourier transform of a distribution, $f$, over the sphere of radius $k$ in momentum space. The incident beam can be defined to be the restriction of $f$ to the hemisphere, $S$, pointing toward the barrier, $k_{z}>0$. To evaluate the incident current, we shall work in spherical coordinates. Once the result is evaluated, the generalisation to arbitrary coordinates will be straightforward. In spherical coordinates, with $\hat{z}$ normal to the barrier, we have the incident beam

$$
\begin{aligned}
\psi_{0} & =\int_{0}^{\pi / 2} \int_{0}^{2 \pi} \mathrm{d} \alpha \mathrm{d} \beta \sin \alpha f(\alpha, \beta) e^{i \boldsymbol{k}(\alpha, \beta) \cdot \boldsymbol{r}}, \\
\boldsymbol{k}(\alpha, \beta) & =k(\cos \alpha \hat{z}+\sin \alpha(\cos \beta \hat{x}+\sin \beta \hat{y})) .
\end{aligned}
$$

The probability current incident on the barrier is given by the imaginary part of

$$
\begin{aligned}
& \int \mathrm{d}^{2} r \psi_{0}^{*} \frac{\partial \psi_{0}}{\partial z} \\
= & i \int \mathrm{d}^{2} r \iint_{0}^{\pi / 2} \mathrm{~d} \alpha \mathrm{d} \alpha^{\prime} \iint_{0}^{2 \pi} \mathrm{d} \beta \mathrm{d} \beta^{\prime} \sin \alpha \sin \alpha^{\prime} f^{*}(\alpha, \beta) f\left(\alpha^{\prime}, \beta^{\prime}\right) k \cos \alpha e^{i\left(\boldsymbol{k}-\boldsymbol{k}^{\prime}\right) \cdot \boldsymbol{r}} .
\end{aligned}
$$

The integral over the $x, y$ plane will give a term

$$
\begin{gathered}
\int \mathrm{d}^{2} r \exp \left(i k x\left[\sin \alpha \cos \beta-\sin \alpha^{\prime} \cos \beta^{\prime}\right]+i k y\left[\sin \alpha \sin \beta-\sin \alpha^{\prime} \sin \beta^{\prime}\right]\right) \\
=\frac{(2 \pi)^{2}}{k^{2}} \delta\left(\sin \alpha \cos \beta-\sin \alpha^{\prime} \cos \beta^{\prime}\right) \delta\left(\sin \alpha \sin \beta-\sin \alpha^{\prime} \sin \beta^{\prime}\right) .
\end{gathered}
$$

This is equivalent to (see 2.7.8 for derivation)

$$
\frac{(2 \pi)^{2}}{k^{2}|J|} \delta\left(\alpha-\alpha^{\prime}\right) \delta\left(\beta-\beta^{\prime}\right)
$$


where $J=\sin \alpha \cos \alpha$ is the determinant of the Jacobian

$$
\left(\begin{array}{cc}
\cos \alpha \cos \beta & \cos \alpha \sin \beta \\
-\sin \alpha \sin \beta & \sin \alpha \cos \beta
\end{array}\right) .
$$

Hence, the current will simply be given by the integral of the modulus of the distribution $f$, over $S$.

$$
\boldsymbol{j}_{0} \cdot \hat{n}=\int_{0}^{\pi / 2} \int_{0}^{2 \pi} \mathrm{d} \alpha \mathrm{d} \beta \sin \alpha|f(\alpha, \beta)|^{2} .
$$

The generalisation to arbitrary coordinates $u, v$ can be obtained simply by noting that $k^{2} \sin \alpha \mathrm{d} \alpha \mathrm{d} \beta$ is the surface area element in spherical coordinates in momentum space, so for arbitrary coordinates $u, v$, we have

$$
\boldsymbol{j}_{0} \cdot \hat{n}=\int_{S}|f(u, v)|^{2} d A .
$$

If $f$ is square integrable, we can normalise for unit incident probability current. The transmittance is then given by

$$
T=\int_{S}|f(u, v)|^{2}|T(u, v)|^{2} d A,
$$

where $A_{T}(u, v)$ is the transmission function in the coordinates $u, v$

$$
A_{T}(u, v)=\frac{k_{z}(u, v)}{k_{z}(u, v)+i Q}
$$

\subsection{Proto-beam oblique transmittance}

The proto-beam in spherical coordinates is

$$
\int_{0}^{\pi / 2} \int_{-\pi}^{\pi} \mathrm{d} \alpha \mathrm{d} \beta \sin \alpha \cos \alpha e^{i \boldsymbol{k}(\alpha, \beta) \cdot \boldsymbol{r}} .
$$

In the barrier normal coordinates, the weight function involves a step function over the region

$$
R=\{(\alpha, \beta): \cos \alpha \cos \theta+\sin \alpha \sin \theta \cos \beta>0\} .
$$


The weight function becomes

$$
f(\alpha, \beta)=\cos \alpha \cos \theta+\sin \alpha \sin \theta \cos \beta
$$

The incident current is given by

$$
j_{0}(\theta)=\int_{R} \mathrm{~d} \alpha \mathrm{d} \beta \sin \alpha(\cos \alpha \cos \theta+\sin \alpha \sin \theta \cos \beta)^{2} .
$$

The transmitted current is therefore given by

$$
j_{T}(Q / k, \theta)=\int_{R} \mathrm{~d} \alpha \mathrm{d} \beta \sin \alpha(\cos \alpha \cos \theta+\sin \alpha \sin \theta \cos \beta)^{2} \frac{\cos ^{2} \alpha}{\cos ^{2} \alpha+Q^{2} / k^{2}}
$$

The transmittance is then given by

$$
T(Q / k, \theta)=\frac{j_{T}(Q / k, \theta)}{j_{0}(\theta)} .
$$

Figure 5.12 shows numerically computed transmittances as a function of $Q$ for incident angles of $\theta=n \pi / 8, n=1,2,3$. Figure 5.11 shows the transmittances variation with $\theta$ for $Q=k$. The variation is similar for other values of $Q$, but scaled inversely proportional to barrier strength.

\subsection{Discussion}

We have seen that if a beam is completely forward propagating relative to a plane, then the integral of probability density over that plane is an invariant. If the beam is not completely forward propagating, the probability density per unit length oscillates in the direction normal to the plane, with the average density being given by the probability densities of the forward and backward propagating components individually.

The reflection and transmission coefficients themselves are derived from a conservation law, that of probability current. This is the cause for the relation

$$
\left|A_{R}(\phi)\right|^{2}+\left|A_{T}(\phi)\right|^{2}=1 .
$$


Due to this conservation law, in addition to the current being conserved, the radial integral of average probability density in a plane parallel to the barrier is conserved. Specifically, adding the total probability density per unit length for both sides of the barrier, and averaging, is equal to twice the incident probability per unit length,

$$
\left\langle\int \mathrm{d}^{2} r\left|\psi_{0}\right|^{2}+\left|\psi_{R}\right|^{2}+\left|\psi_{T}\right|^{2}\right\rangle=2\left\langle\int \mathrm{d}^{2} r\left|\psi_{0}\right|^{2}\right\rangle
$$

We have restricted our discussion to the Dirac delta potential well. A simple generalisation would be to the step potential, which has the main difference of having a different wave-number on either side of the barrier. This is a more realistic model for most systems, as it is rare to have a thin barrier without a change in propagation characteristics on either side.

For both the Delta potential and step potential, the barrier is taken to be infinitely large, and as such the reflection of the beam depends only on the global properties, not the local properties. As such, while the zeros are deflected by the barrier, the transmittance is unaffected by the position of the focus relative to the barrier. At this stage we are unsure whether this is entirely due to modelling the barrier as infinite, or due the characteristics of scalar beams. We suspect that for an apertured barrier the local properties would be more important, however such systems are highly unlikely to be symbolically tractable, and would most likely need to be investigated numerically.

Our analysis is also restricted to scalar beams. For electromagnetic beams the polarisation of the beam plays an important role. In Chapter 4 we saw that the zeros of a wavefunction are associated with polarisation singularities in electromagnetic beams. It would be interesting to see if this would affect the reflection characteristics.

Finally, we note that shifting the location of the focal plane relative to the barrier results in dramatic changes in the topology of the phase surfaces, much like shifting the focal parameter of the Carter wavefunction did in Chapter 4 . For particle beams, these are associated with the creation of ring vortices of particle current, and for electromagnetic beams, polarisation singularities. This 
dramatic topological change is a good candidate for a property of the system which could be investigated experimentally. 


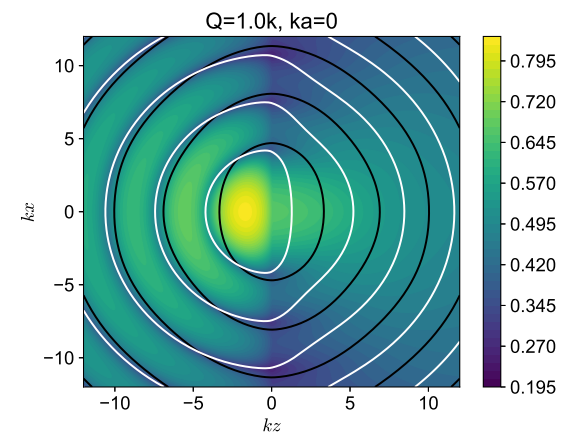

(a)

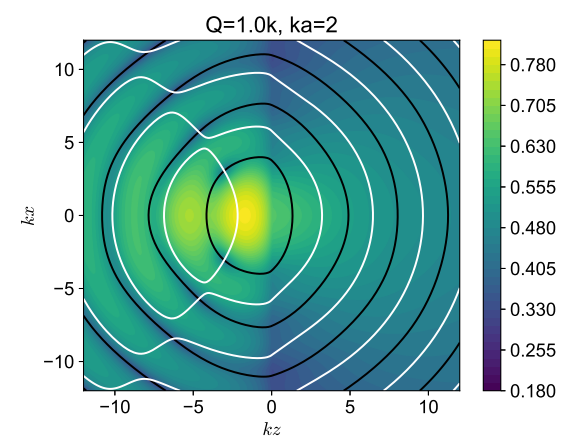

(c)

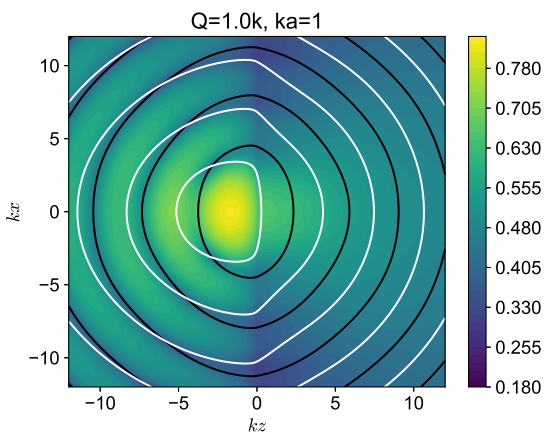

(b)

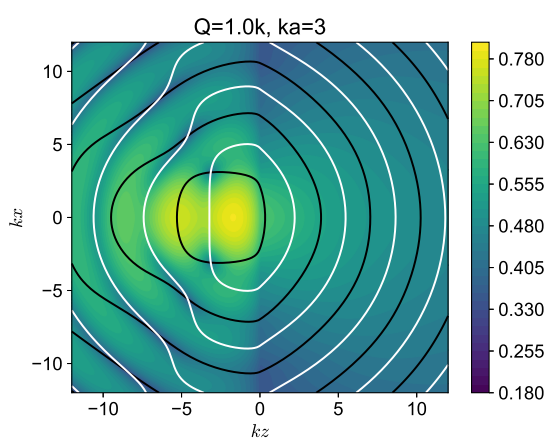

(d)

Figure 5.5: Comparison of proto-beam translated by $k a=0,1,1,2$, for barrier strength $Q=k . k a>0$ shifts the focal plane into the incident side. Between $k a=2,3$ there is a topological change of the phase surfaces in the incident region, that results in the creation of new zeros. This is reminiscent of the Carter wavefunction for Chapter 4 


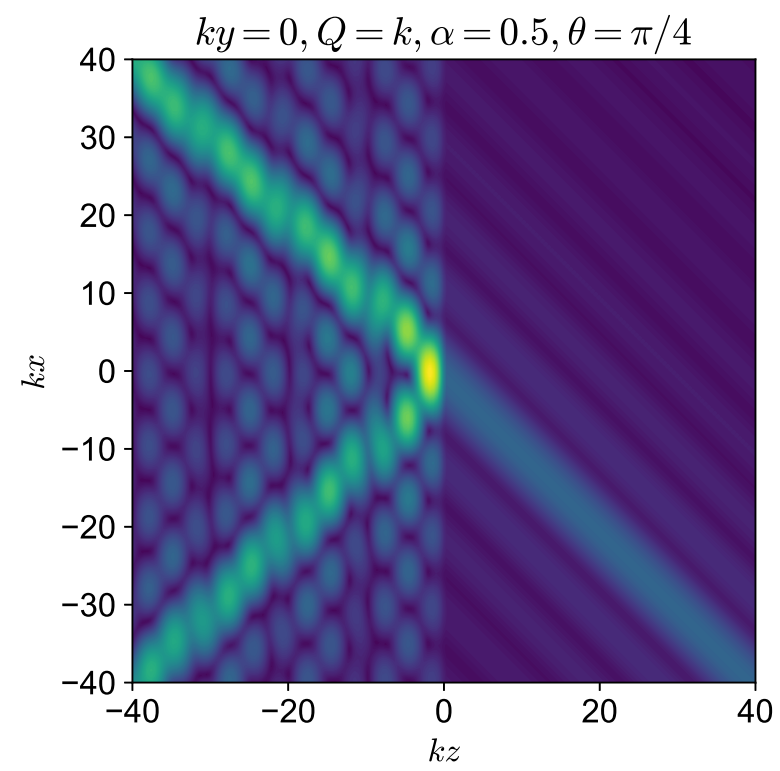

Figure 5.6: Bessel beam with conical angle of 0.5 radians, incident at $45^{\circ}$ angle to potential barrier. Shading proportional to probability density, with barrier visible at the abrupt transition at $k z=0$.

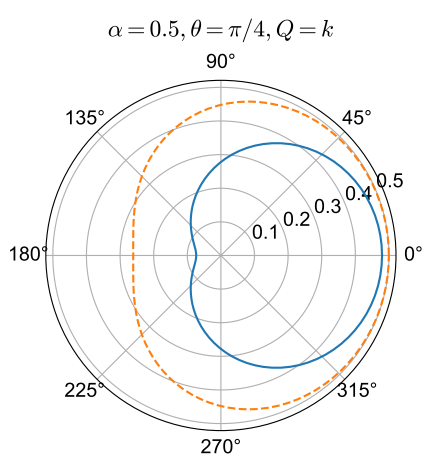

(a)

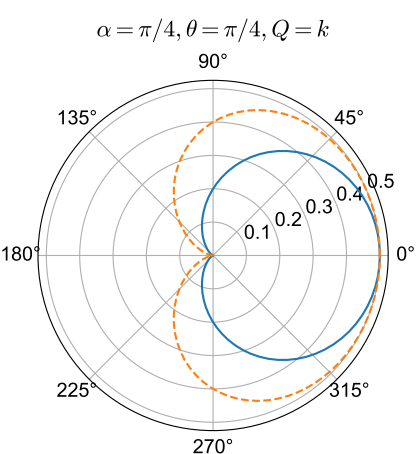

(b)

Figure 5.7: Polar plots of real (solid, blue), and imaginary (dashed, orange) parts of $A_{T}(\phi)$ for an incident Bessel beam. Note the reduction in amplitude of components propagating toward the barrier $\phi=\pi$. Also note the effect of varying the conical angle $\alpha$. 

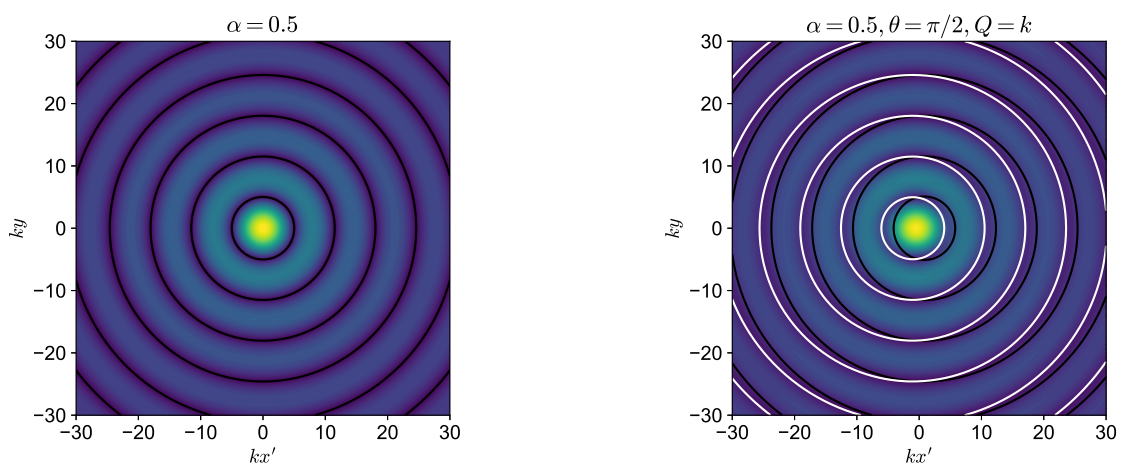

Figure 5.8: Radial wave-function modulus for incident (left) and transmitted (right) Bessel beams with a conical angle of 0.5 radians. Marked are the real (black) and imaginary (white) zero contours. For the incident beam the imaginary part is uniformly zero (hence why white contours are not shown), whereas for the transmitted beam the imaginary part has ring zeros, that are offset from the real zeros. At the intersections there is an associated phase singularity, with the probability current circulating. 


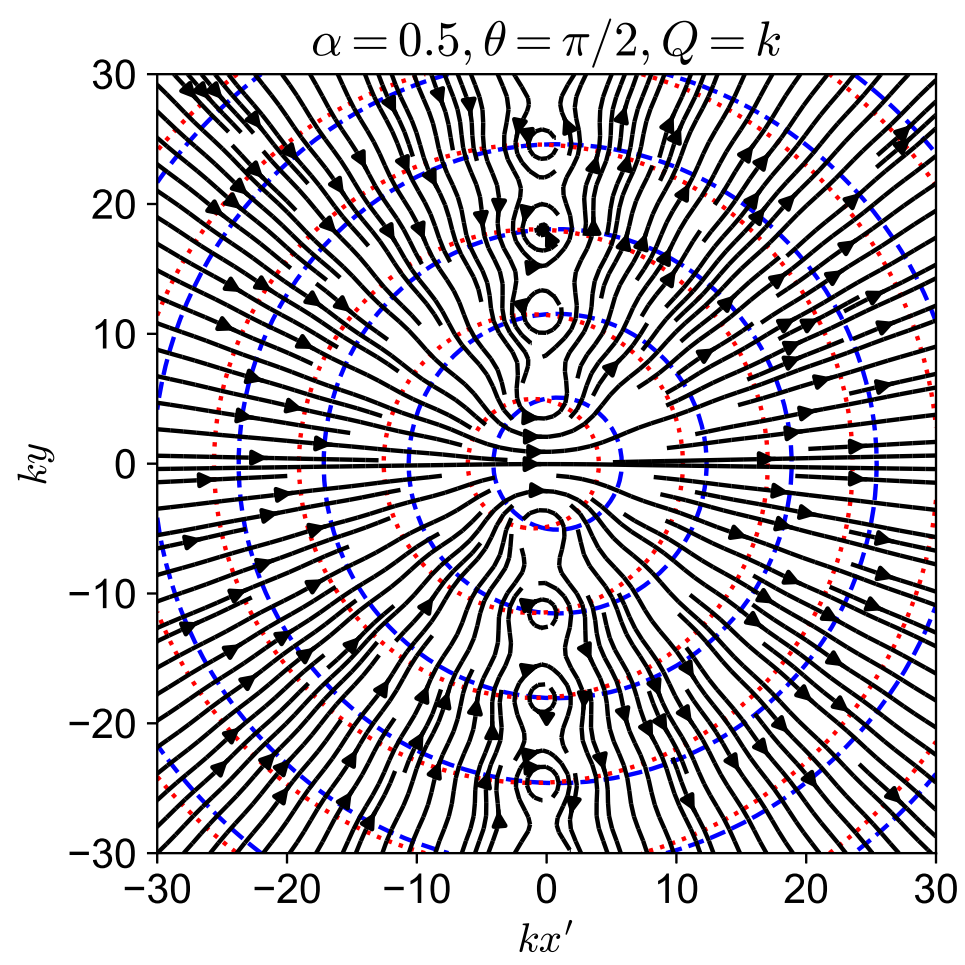

Figure 5.9: Probability current streamlines in radial plane for transmitted Bessel beam, with real (blue, dashed) and imaginary (red, dotted) zero contours in background. Note the associated circulation around the wave-function zeros, located at crossing of zero contours. Also note the net probability current flow in the direction of increasing $x^{\prime}$, away from the barrier. 


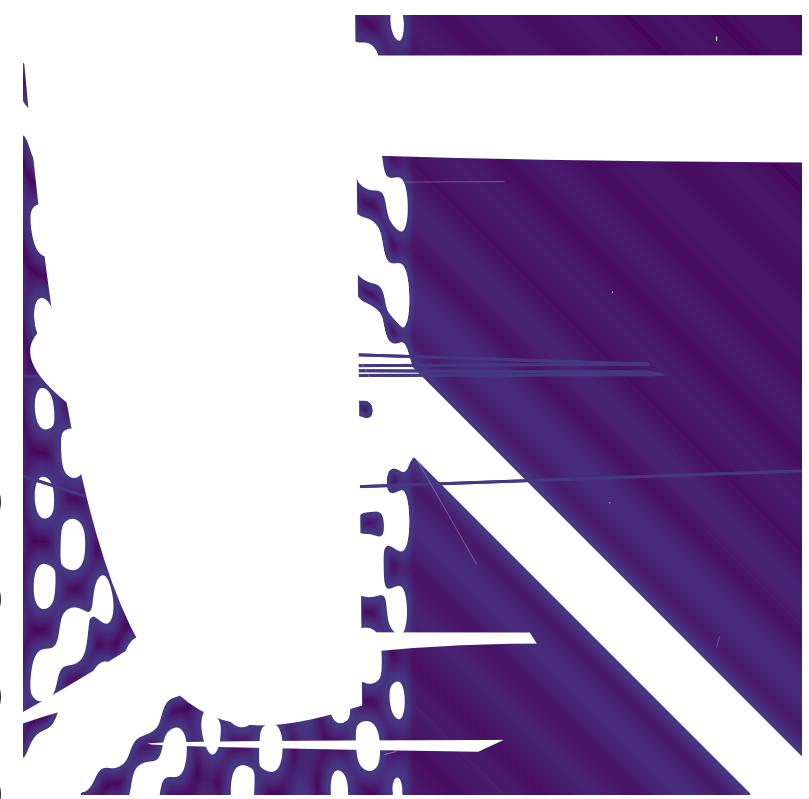

Figure 5.10: Probability current streamlines for Bessel beam in plane of incidence. 


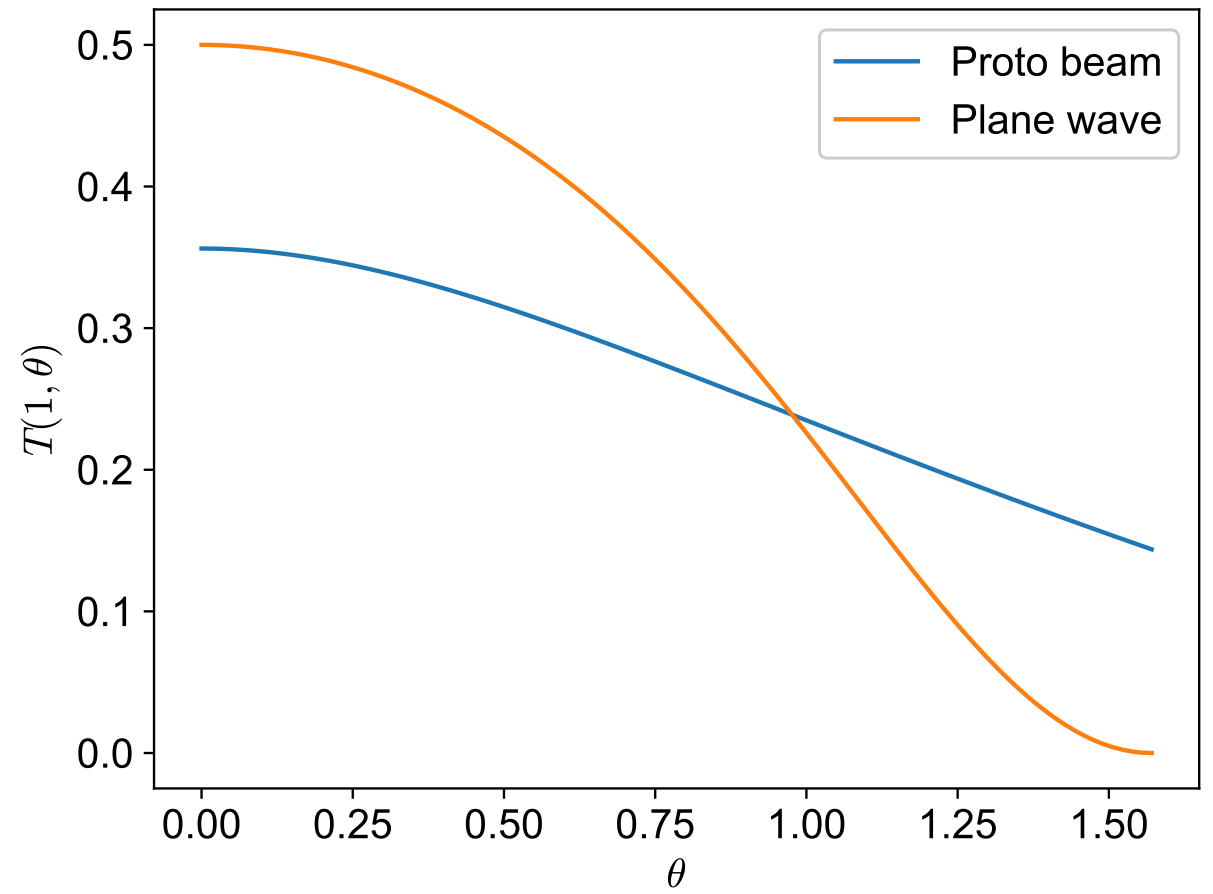

Figure 5.11: Comparison of transmittance as a function of incident angle (in radians) for both proto-beam and a plane wave, on the $Q=k$ barrier. The plane wave begins with a higher transmittance, but past a certain angle the protobeam overtakes it. While a plane wave is totally reflected at grazing incidence, the proto-beam is not. This is because the proto-beam contains a superposition of plane waves over an entire hemisphere in momentum space, and when the beam is at grazing angles some of these plane wave components will still be incident on the barrier. 


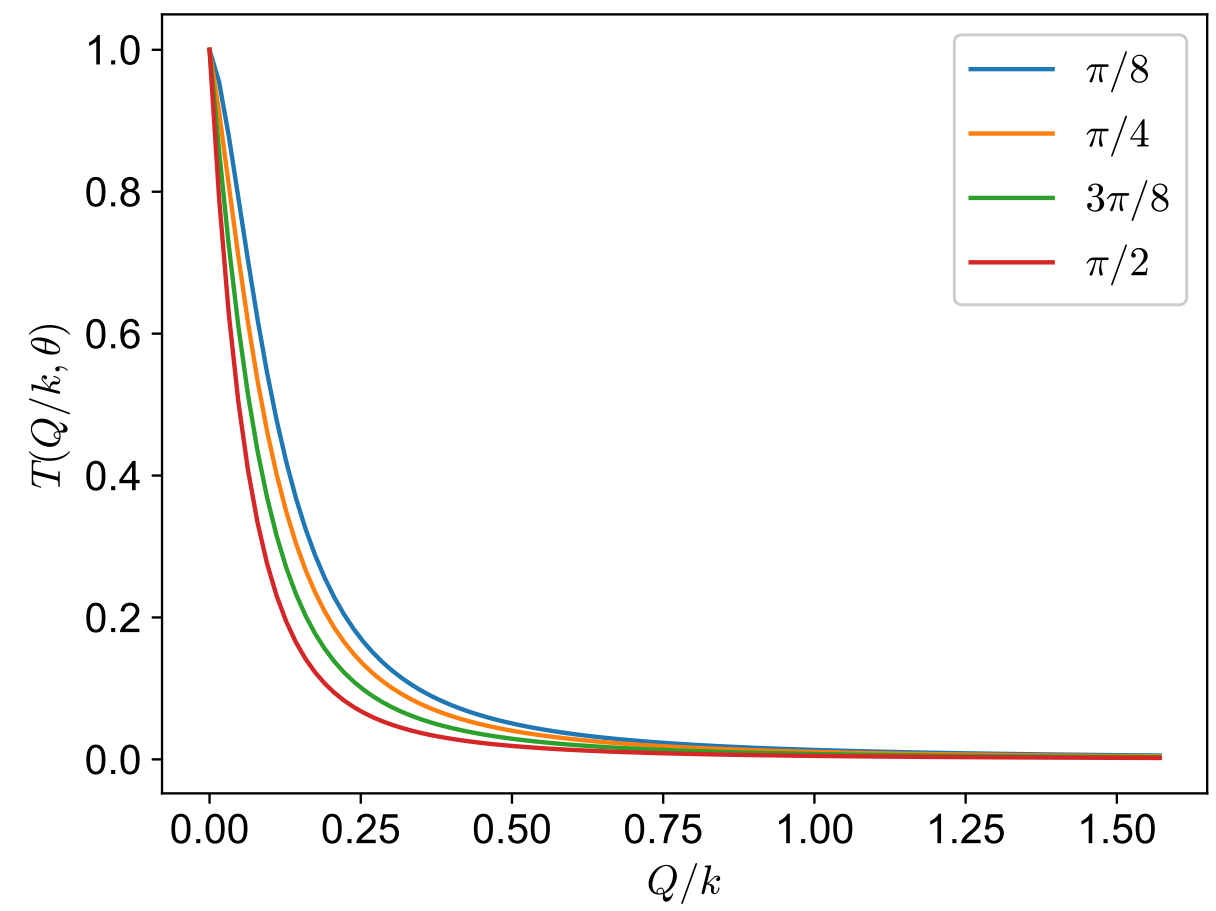

Figure 5.12: Transmittance as a function of barrier strength for various angles of incidence of the proto-beam. The transmittance profiles are similar, with an overall decrease toward grazing angles. 


\section{Chapter 6}

\section{Lorentz transformation of monochromatic beams}

\subsection{Introduction}

The Doppler effect is the shift in frequency of a wave when an observer changes velocity relative to the source. The derivation for the Doppler effect can be found in many textbooks [see Jackson, 18, section 11.3], however we shall provide one here for illustrative purposes. Consider a monochromatic plane wave, travelling along $z$. The wave has the form

$$
\psi(z, t)=\exp (i[k z-\omega t]), \quad \omega=\omega(k) .
$$

Under a Lorentz boost, the old coordinates in terms of the new coordinates are given by

$$
\begin{aligned}
& z=\gamma\left(z^{\prime}-\beta c t^{\prime}\right), \\
& t=\gamma\left(t^{\prime}-\beta \frac{z^{\prime}}{c}\right) .
\end{aligned}
$$

Note that positive $\beta$ corresponds to a boost away from the propagation direction, toward the source. Thus, the plane wave in the new coordinates is given by

$$
\psi^{\prime}\left(z^{\prime}, t^{\prime}\right)=\psi\left(z\left(z^{\prime}, t^{\prime}\right), t\left(z^{\prime}, t^{\prime}\right)\right)=\exp \left(i\left[k \gamma\left(z^{\prime}-\beta c t^{\prime}\right)-\omega \gamma\left(t^{\prime}-\beta \frac{z^{\prime}}{c}\right)\right]\right) .
$$


We can rearrange this into the suggestive form

$$
\psi^{\prime}\left(z^{\prime}, t^{\prime}\right)=\exp \left(i \gamma\left[z^{\prime}\left(k+\beta \frac{\omega}{c}\right)-t^{\prime}(\omega+\beta k c)\right]\right) .
$$

The result is a plane wave with new wave-vector and frequency

$$
\begin{aligned}
k^{\prime} & =\gamma\left(k+\beta \frac{\omega(k)}{c}\right), \\
\omega^{\prime} & =\gamma(\omega(k)+\beta k c) .
\end{aligned}
$$

This gives a ratio of

$$
\frac{\omega^{\prime}}{\omega}=\gamma\left(1+\beta \frac{k(\omega) c}{\omega}\right) .
$$

For a massless particle, the dispersion is $\omega=k c$, thus we have the Doppler ratio

$$
\frac{\omega^{\prime}}{\omega}=\sqrt{\frac{1+\beta}{1-\beta}} .
$$

Note that the beam was monochromatic in the original frame, and after the boost is still monochromatic in the boosted frame. The frequency is red-shifted for motion away from the source, and blue-shifted for motion toward.

If the motion is not in the direction of the wavevector, but rather at an angle $\theta$ in the $\hat{x}$ direction, the Doppler shift for a light-like wave is given by [Jackson, 18, Section 11.3]

$$
\frac{\omega^{\prime}}{\omega}=\frac{1+\beta \cos \theta}{\sqrt{1-\beta^{2}}} .
$$

The magnitude of the Doppler shift will be lessened, and the wavevector will be tilted away from the observer. We shall not go into detail here as the scope of this thesis is limited to longitudinal shifts.

Plane waves are only one choice of basis function for solutions of the wave equation. The basis for cylindrically symmetric monochromatic beams are the Bessel-beams,

$$
\psi(\rho, \phi, z)=e^{i(q z-\omega t+m \phi)} J_{m}(\kappa \rho), \quad q^{2}+\kappa^{2}=k^{2}=\frac{\omega^{2}}{c^{2}}, \quad m \in \mathbb{Z} .
$$

For a given beam, $q, \kappa, m$ are fixed. Under a Lorentz boost, the new frequency is given by

$$
\omega^{\prime}=\gamma(\omega+\beta q(\omega))
$$


Now since $q=\sqrt{\omega^{2} / c^{2}-\kappa^{2}}$, the Doppler factor is given by

$$
\frac{\omega^{\prime}}{\omega}=\gamma\left(1+\beta \sqrt{1-\frac{\kappa^{2} c^{2}}{\omega^{2}}}\right) .
$$

Note however, that the resulting beam is still not dispersive: the Doppler shift is smaller than the plane wave case, but the resulting beam is still monochromatic. This resembles the Doppler shift of a massive particle following the Klein-Gordon equation, with mass of $\hbar \kappa c$. This is due to the propagation characteristics of Bessel beams, which have an axial phase velocity slower than $c$. Physical Bessel beams occur only as wave-guide modes; they are not square integrable radially, which is a necessary condition for beam invariants to be finite (see Section 2.2). It is well established that wave guide modes have phase velocities slower than plane waves in free space (see for example [Griffiths, 28. section 9.5]).

It follows that one can find a frame where the phase velocity is zero. Further boosting will reverse the observed phase velocity, allowing one to 'overtake' the beam, in the sense of reversing the phase velocity, even for a massless wave. In a plane wave basis Bessel beams are a superposition of plane waves with wavevectors along a cone, as noted in the introduction (Chapter 1). Boosting 'flattens' the cone closer to a disk, and in the zero phase velocity frame all plane wave components lie in a disk. The propagation of each component cancels out the component with the opposite momentum, and the beam is perceived to be 'stationary' or 'frozen', with no time variation in phase.

\subsection{Generalised Bessel Beam}

While Bessel beams have interesting Lorentz transformation characteristics, much of this is due to the fact they are eigenmodes of $\hat{k}_{z}$, much like plane waves are. Much like plane waves however, they are physical, and less relevant as well, as unlike plane waves, local propagation will not be described by Bessel beams. We instead turn our attention to general, azimuthally symmetric, forward prop- 


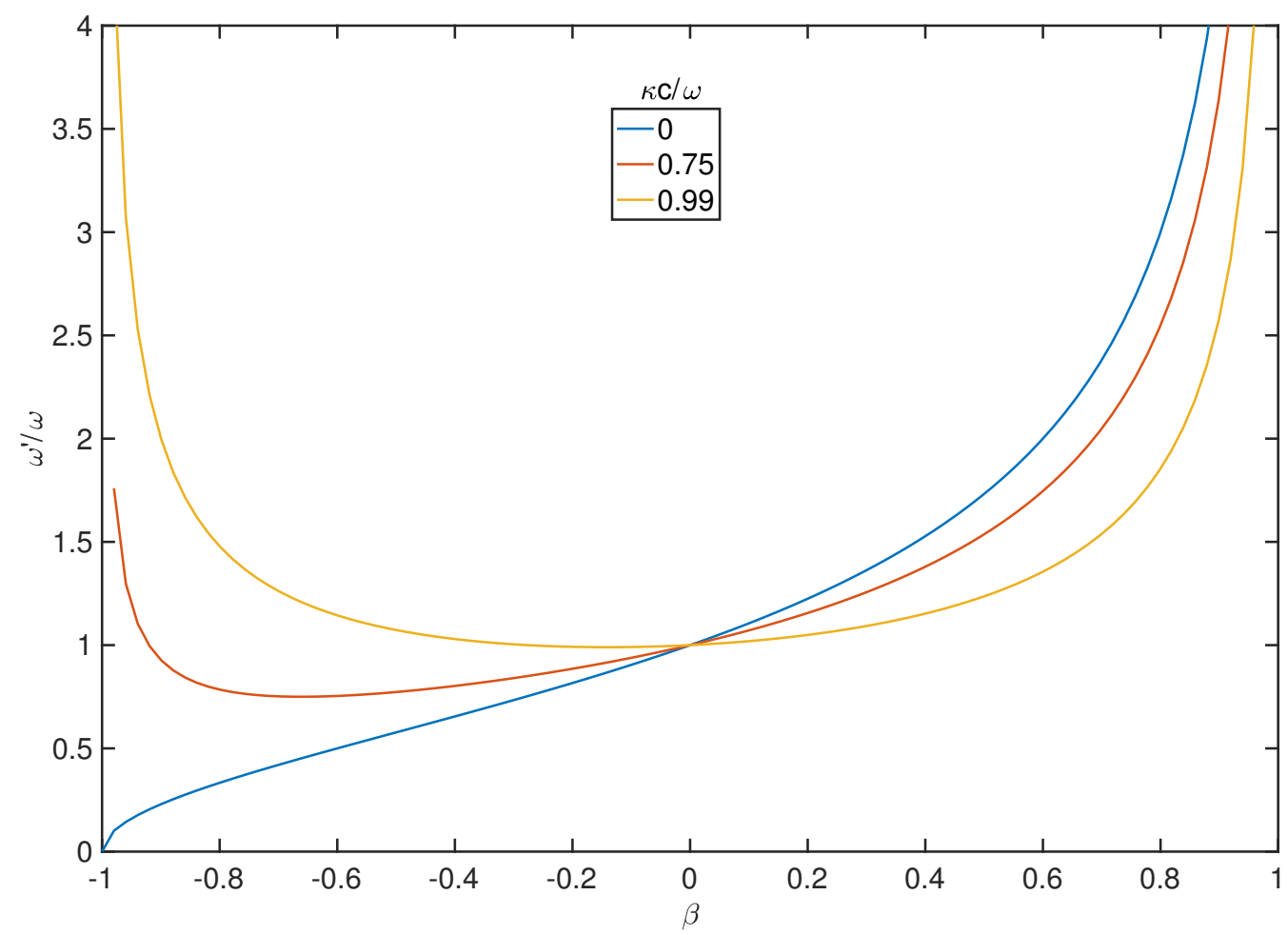

Figure 6.1: Bessel beam Doppler ratios for various values of $\kappa c / \omega$. For large enough $\beta$ there will be a blue-shift even when moving away from the source. This is because Bessel beams, being waveguide modes, have a phase velocity slower than $c$, thus it is possible to "overtake" the beam, so that the phase velocity changes direction.

agating beams, which can be constructed as superpositions of Bessels,

$$
\psi(\rho, z, t)=e^{-i \omega t} \int_{0}^{k} \mathrm{~d} \kappa e^{i q z} J_{0}(\kappa \rho) f(k, \kappa), \quad q^{2}+\kappa^{2}=k^{2}=\frac{\omega^{2}}{c^{2}} .
$$

A Lorentz boost along $z$ gives

$$
\frac{\omega^{\prime}(\kappa)}{\omega}=\gamma\left(1+\beta \frac{q c}{\omega}\right)
$$

as before. However, for a Bessel beam, there is only one value for $q$, and there will be only one frequency present in the transformed beam (the distribution 
will be a Dirac delta). For an arbitrary beam, as there is a dispersion in $q$, there is also a dispersion in $\omega^{\prime}$. Inverting, we have

$$
q\left(\omega^{\prime} / \omega\right)=\frac{\omega}{c} \frac{\omega^{\prime} / \omega-\gamma}{\beta \gamma} .
$$

As the range of $q$ is $\left(0, \frac{\omega}{c}\right)$, care must be chosen to suitably restrict the range of $\omega^{\prime}$ when evaluating the dispersion; the range of $\omega^{\prime}$ will be $\left(\gamma \omega, \omega_{D}\right)$ for positive $\beta$, and $\left(\omega_{D}, \gamma \omega\right)$ for negative $\beta$, with $\omega_{D}(\beta)$ the plane wave Doppler frequency given by 6.1.7). The physical reason for this range in $\omega^{\prime}$ is clear from the plane wave Doppler shift: the maximum is for the components travelling parallel to the boost direction, and are shifted by the full shift. The lower limit is for the components travelling perpendicular to the beam axis, which are are shifted by the transverse Doppler shift of $\gamma$. The dispersion in $\omega^{\prime}$ will then be given by

$$
\bar{f}\left(\omega^{\prime} / \omega\right)=f\left(q\left(\omega^{\prime} / \omega\right)\right) .
$$

Figure 6.2 gives two examples of dispersions for a motion of $\beta=0.2$ toward the source.

\subsection{Lorentz transformation of wave envelopes}

Consider expressing a beam in envelope-phase formulation,

$$
\psi=e^{i(k z-\omega t)} u(\boldsymbol{r}, t)
$$

Note that the possible time dependence in the envelope: while an observer stationary relative to the source will see a stationary envelope, an observer who is moving relative to the source will see a moving envelope. When we apply the wave equation to this beam, we see that the resulting differential equation for the envelope is

$$
\square u+2 i\left(k \frac{\partial u}{\partial z}+\frac{\omega}{c} \frac{\partial u}{\partial t}\right)+\left(\frac{\omega^{2}}{c^{2}}-k^{2}\right) u=0 .
$$

In the case of a luminal beam, we have $k=\frac{\omega}{c}$, so this gives us

$$
\square u+2 i k\left(\frac{\partial u}{\partial z}+\frac{1}{c} \frac{\partial u}{\partial t}\right) u=0 .
$$




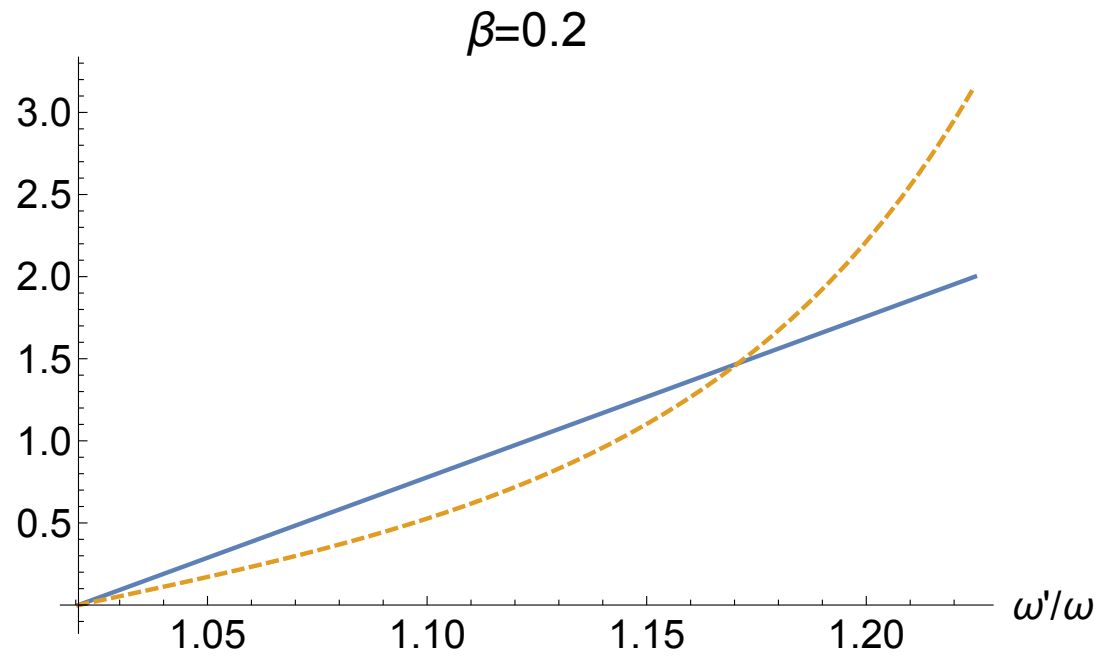

Figure 6.2: Frequency dispersions for a motion with $\beta=0.2$ toward the source. The blue, solid curve is the proto-beam, and the orange, dashed the $k b=2$ Carter wavefunction from Chapter 4 .

We can put this equation into relativistic form by defining the light-like wavevectors

$$
k_{+}^{\mu}=k(1,0,0,1), k_{-}^{\mu}=k(-1,0,0,1) .
$$

The expression for the beam and the resulting differential equation become

$$
\begin{gathered}
\psi=e^{i k_{+\mu} x^{\mu}} u(x), \\
\square u+2 i k_{-}^{\mu} \partial_{\mu} u=0 .
\end{gathered}
$$

Now we examine the form of the equation under Lorentz transformations. First we boost along $\mathrm{z}$. The wavevectors in the new frame are

$$
\begin{aligned}
& \bar{k}_{+}^{\mu}=\gamma k(1-\beta, 0,0,1-\beta)=\sqrt{\frac{1-\beta}{1+\beta}} k_{+}^{\mu}, \\
& \bar{k}_{-}^{\mu}=\gamma k(-1-\beta, 0,0,1+\beta)=\sqrt{\frac{1+\beta}{1-\beta}} k_{-}^{\mu} .
\end{aligned}
$$

Thus, we can see that the familiar result of the phase being Doppler shifted as a plane wave. However, the envelope is inverse Doppler shifted. This is because 
the envelope consists of counter-propagating corrections to the plane wave. A moving observer will see a time dependent envelope, which is not possible with a monochromatic beam. In many cases, in the rest frame the envelope varies over a distance of many wavelengths. As such, in the transformed frame, the envelope will vary over a time of many periods. Recall that the local frequency is given by -1 times the time derivative of the phase. In many experiments, particularly those where we can treat particles as point-like, it is the local properties of the wave that are important. In the new frame, the envelope times phase can be written as

$$
\psi\left(x, y, z^{\prime}, t^{\prime}\right)=u\left(x, y, z^{\prime}, t^{\prime}\right) e^{i\left(k_{D} z^{\prime}-\omega_{D} t^{\prime}\right)}
$$

with $k_{D}$ and $\omega_{D}$ the plane wave Doppler shifted wave vector and frequency. The phase of the wave is given by

$$
S\left(x, y, z^{\prime}, t^{\prime}\right)=S_{u}\left(x, y, z^{\prime}, t^{\prime}\right)+k_{D} z^{\prime}-\omega_{D} t^{\prime},
$$

with $S_{u}$ the phase of the envelope. The local frequency is therefore

$$
\omega_{0}=\omega_{D}-\frac{\partial S_{u}}{\partial t}
$$

As long as $\frac{\partial S_{u}}{\partial t} \ll \omega_{D}$, we are safe to use the the plane wave Doppler shift in our calculations.

\subsection{Paraxial beams}

In its usual form, the paraxial wave equation 6.4.7 is not explicitly Lorentz covariant. Given a solution of (6.4.10), the solution will not satisfy the paraxial wave equation in a Lorentz transformed coordinate system. Bélanger [49, 50] examines Gaussian packet-like exact solutions to the wave equation, which can be unambiguously Lorentz transformed as the wave equation is covariant.

$$
G(\rho, \phi, z, c t)=F(\rho, \phi, \gamma(z+\beta c t)) \exp \left(-\frac{i k(z-\beta c t)}{2}\right)
$$


At this stage, $\beta, \gamma$ are not yet identified with their usual relativistic interpretation, and are merely a pair of free parameters. We note there are numerous errors in Bélanger, 1986 [50]. The first of which is the differential equation for $F$; Bélanger claims that it satisfies

$$
\nabla_{\perp}^{2}-i k \gamma(1+\beta) \frac{\partial F}{\partial z_{c}}+\gamma^{2}\left(1-\beta^{2}\right) \frac{\partial^{2} F}{\partial z_{c}^{2}}, \quad z_{c} \equiv \gamma(z+\beta c t) .
$$

The actual differential equation for the envelope can be easily verified using computer algebra systems and is given by

$$
\frac{k^{2}\left(\beta^{2}-1\right)}{4} F+\nabla_{\perp}^{2} F-i k \gamma\left(1+\beta^{2}\right) \frac{\partial F}{\partial z_{c}}+\gamma^{2}\left(1-\beta^{2}\right) \frac{\partial^{2} F}{\partial z_{c}^{2}}
$$

The assertion that when $\beta \rightarrow 1$ the equation becomes the paraxial wave equation with $z \rightarrow z_{c}$ is correct however. With this choice of parameter, and setting $\gamma \rightarrow 1$, Bélanger's Guassian packet solution is indeed an exact solution of the wave equation,

$$
F(\rho, \phi, z+\beta c t)=\frac{1}{\xi} \exp \left(-\frac{i \rho^{2}}{2 \xi}\right), \quad \xi \equiv z+c t+i \frac{k b^{2}}{2}
$$

It is our view that such a solution is simply not physical. The centre of intensity of the packet travels at $c$. This in itself is not problematic, however, the packet is not square integrable over all space, at any time. For beams this is not an issue as the beam originates at a source, and in the sources rest frame the beam has time independent cycle average quantities, such as energy or momentum density. As such, for a beam it is sufficient if cycle average quantities per unit length are finite. For the Bélanger packet the source would travel at $c$, which is not physical as any physical source is massive. Such solutions can be analytically useful however, for example Ziolkowski [51, 52] uses superpositions of these solutions with different values of $k$ and $b$ to construct physical (square integrable) solutions.

Bélanger then claims that under a Lorentz transformation, the paraxial Gaussian beam will approach the exact packet-like solution (6.4.4). We take issue with this treatment as first, Bélanger has numerous errors in his analysis, and 
furthermore it does not make it clear how the paraxial approximation should be treated in a different frame; Bélanger seems to suggests that the approximate solution should approach an exact solution. We do not think this is the case, and believe the paraxial approximation should in fact be made in a relativistically covariant way.

We shall use the approach derived independently by Wünsche [53], Longhi [54], Besieris and Shaarawi [55]. The usual paraxial approximation first begins with a monochromatic wave

$$
e^{-i \omega t} \psi(x, y, z)
$$

The spatial dependence is the assumed to be envelope-phase form,

$$
\psi(x, y, z)=e^{i k z} u(x, y, z) .
$$

The Helmholtz equation for the spatial dependence is then modified under the assumption $\frac{\partial^{2} u}{\partial z^{2}} \ll k^{2} u$, which is the assumption that the envelope varies over a length scale far greater than the wavelength. The paraxial equation for the envelope is therefore

$$
\nabla_{\perp}^{2} u+2 i k \frac{\partial u}{\partial z}
$$

the familiar form seen in the introduction. The issue is in assuming the envelope has no time dependence: this is only true in the rest frame of the source. A moving observer will see a moving envelope, thus we must include a time dependence in general.

We propose the following generalisation: write the wave in the following form

$$
\psi(x, y, z, t)=e^{i k_{\mu}^{+} x^{\mu}} u(x, y, z, t), \quad k^{+\mu}=k(1,0,0,1) .
$$

Now assume that the envelope varies over both length scales and time scales much larger than the wavelength and period of the phase factor,

$$
\frac{\partial^{2} u}{\partial z^{2}} \ll k^{2} u, \quad \frac{\partial^{2} u}{\partial t^{2}} \ll c^{2} k^{2} u .
$$

We therefore neglect the second derivatives in both $z$ and $t$ in the differential equation for the envelope. The envelope will then satisfy the equation

$$
\nabla_{\perp}^{2} u+2 i k^{-\mu} \partial_{\mu} u=0, \quad k^{-\mu}=k(-1,0,0,1) .
$$


It is easy to see that if the envelope is time independent this reduces to the regular paraxial equation. This equation is also relativistic: we can write the perpendicular Laplacian as

$$
\nabla_{\perp}^{2}=\square-\frac{1}{k^{2}}\left(k^{+\mu} \partial_{\mu}\right)\left(k^{-\mu} \partial_{\mu}\right)
$$

This clearly transforms as a Lorentz scalar operator, and hence the entire operator that acts on $u$ in 6.4 .10 transforms as a Lorentz scalar operator. The symmetry that is broken by the paraxial equation is not Lorentz invariance, as the paraxial assumption can be put into relativistic form. Rather, the wave equation is isotropic, but the paraxial equation breaks this by picking a preferred wavevector. This should not be surprising however, as the preferred wavevector is simply chosen by the beam propagation direction.

This relativistic operator permits Gaussian solutions, however with a possibly time dependent envelope:

$$
u_{G}(\rho, z, t)=\frac{b}{b+i(f z+g c t)} \exp \left(-\frac{k \rho^{2}}{2(b+i(f z+g c t))}\right), \quad f+g=1 .
$$

The constraint $f+g=1$ is necessary to satisfy 6.4 .10 . We can attempt to boost to a frame where this beam is static: under a Lorentz transformation we have

$$
f z+g c t \rightarrow \gamma(z(f+g \beta)+c t(g+f \beta)) .
$$

For this to be independent of $t$, we must have

$$
g=-f \beta
$$

The constraint $f+g=1$ gives

$$
f(1-\beta)=1
$$

This can be solved to give

$$
\beta=\frac{f-1}{f} .
$$

This is a valid boost for $f \in[1 / 2, \infty)$, but invalid otherwise. This implies that if $f<1 / 2$, the 'beam' has no rest frame. Such a solution is physically questionable, as it would represent a pulse-like solution, yet the total intensity over 
all space is unbounded. Furthermore, the 'centre of intensity', defined by $f z+$ $g c t=0$, travels with a velocity of

$$
v_{C E} / c=\frac{g}{f}=\frac{1-f}{f}
$$

It is clear that a boost to a rest frame does not exist iff. the beam's centre of intensity travels superluminally. It is our view that this is not physical. We note that

In the previous section we saw how envelope-phase beams transformed, with the envelope transforming with the inverse Doppler shift, as they are composed of counter-propagating corrections. In the new frame, the paraxial equation is given by

$$
\nabla_{\perp}^{2} u+2 i k \sqrt{\frac{1+\beta}{1-\beta}}\left(\partial_{\bar{z}}+\partial_{\bar{c} t}\right) u=0 .
$$

Note how the equation is no longer time independent, and also the occurrence of the inverse Doppler factor.

\subsection{Scattering considerations}

The local wavevector can be defined as the four-gradient of the phase of a wavefunction. As the phase is a Lorentz scalar, the local wavevector must therefore transform as a covector. As a result, there will be local Doppler shift when a beam is transformed. The local wavevector of a beam will differ in magnitude from the nominal wavevector $k=\frac{\omega}{c}$, with the wavefronts being further apart near the focus, and closer together near a zero, compared to the wavefronts far from the focus.

In the source frame, the beam envelope is stationary, with an $e^{-i \omega t}$ time dependent phase, thus the local wavevector and local frequency are independent of time. The local wavevector will however vary spatially. We can thus write the local wavevector at a point $x$ as

$$
\xi^{\mu}=\left(\omega / c, \xi_{x}(\boldsymbol{r}), \xi_{y}(\boldsymbol{r}), \xi_{z}(\boldsymbol{r})\right)
$$


If we now boost along $z$, the new local wavevector will be given by

$$
\bar{\xi}^{\mu}=\left(\gamma\left(\omega / c-\beta \xi_{z}\right), \xi_{x}, \xi_{y}, \gamma\left(\xi_{z}-\beta \omega / c\right)\right)
$$

In addition to varying spatially, each component now varies with time as well, and the local frequency is no longer constant in time, or spatially. Gaussian beams have a simple form, with a closed form expression available for the phase. We shall now examine the local wavevector of a paraxial Gaussian beam, and how it transforms under Lorentz transformations.

Recall once again the equation for a Gaussian beam,

$$
\psi_{G}(\rho, z, t)=\frac{b}{b+i z} \exp \left(i k(z-c t)-\frac{k \rho^{2}}{2(b+i z)}\right) .
$$

If we split this into modulus phase form, we obtain

$$
\psi_{G}(\rho, z, t)=\frac{b}{\sqrt{b^{2}+z^{2}}} e^{-\frac{k b \rho^{2}}{2\left(b^{2}+z^{2}\right)}} \exp \left(i k(z-c t)+i \frac{k z \rho^{2}}{2\left(b^{2}+z^{2}\right)}-i \arctan \frac{z}{b}\right) .
$$

Thus the phase is given by

$$
S(\rho, z, t)=k z\left(1+\frac{\rho^{2}}{2\left(b^{2}+z^{2}\right)}\right)-k c t-\arctan \frac{z}{b},
$$

and the local wavevector by

$$
\xi^{\mu}=(k, 0,0, k)+\frac{1}{b^{2}+z^{2}}\left(0, k z \rho \cos \phi, k z \rho \sin \phi, \frac{k \rho^{2}\left(b^{2}-z^{2}\right)-2 b\left(b^{2}+z^{2}\right)}{2\left(b^{2}+z^{2}\right)}\right) .
$$

Of particular interest however is the $\xi_{z}$ component: under a Lorentz transformation this term will result in a significant deviation of the local frequency close to the focus, even for a boost along $z$. We note that the local wavevector can be written as the plane wave phase, plus the deviation term coming from the envelope. The plane wave phase will Doppler shift as expected, and far from the focal plane, $k z \gg 1$, the envelopes contribution will be vanishingly small. Thus, far from the focus the local wavevector transforms as a plane wave, and the Doppler shift will be the usual one. The local frequency along the beam axis 


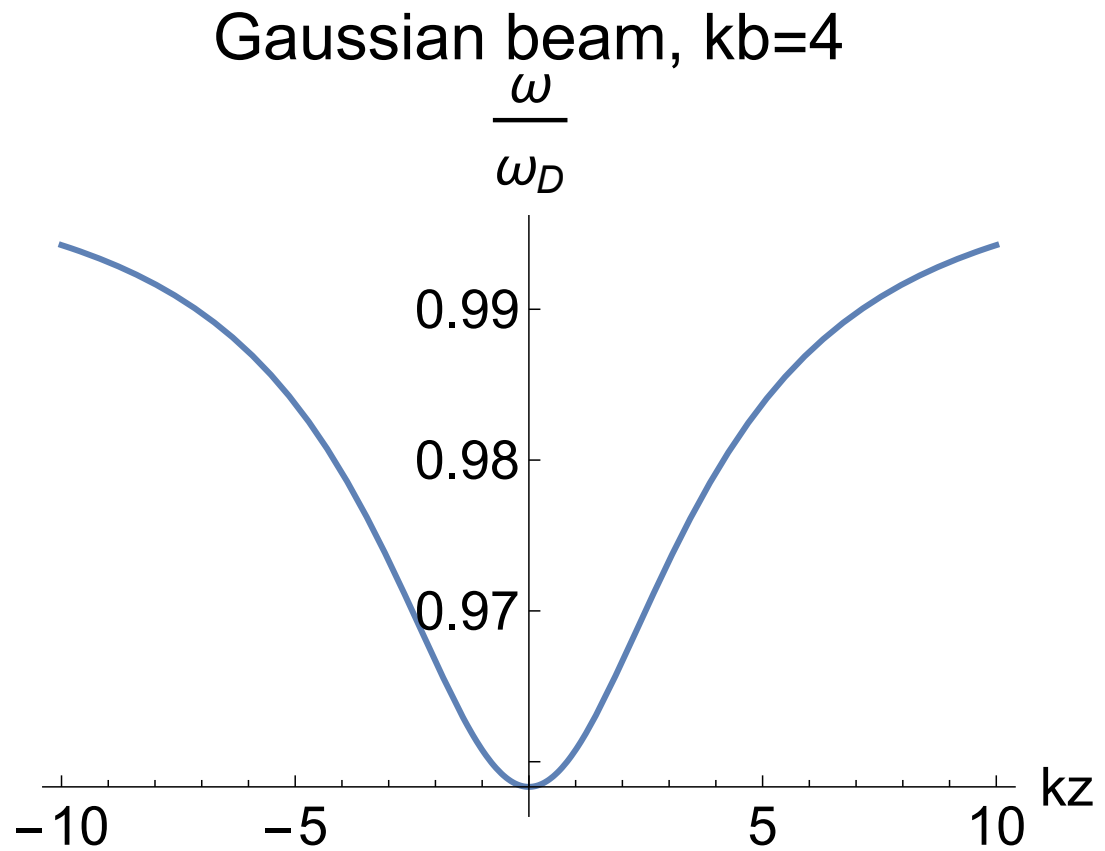

Figure 6.3: Ratio of local frequency to plane wave Doppler shifted frequency for the Gaussian beam with $k b=4$, along the beam axis. The deviation from the plane wave Doppler shift is due to the elongation of the wavelength near the focus.

is given by

$$
\frac{\omega(z)}{c}=\frac{1+\beta\left(1-\frac{b}{k\left(b^{2}+z^{2}\right)}\right)}{\sqrt{1-\beta^{2}}} .
$$

Figure 6.3 gives a plot of the local frequency divided by the plane wave Doppler frequency along the beam axis for $k b=4$.

For general beams, we can find the local frequency along the beam axis easily. Along the beam axis the wavefunction is given by

$$
\psi(0, z)=\int_{0}^{k} f(k, q) e^{i q z}
$$

The phase is given by

$$
S(0, z)=\arctan \left(\frac{\int_{0}^{k} f(k, q) \cos q z}{\int_{0}^{k} f(k, q) \sin q z}\right) .
$$




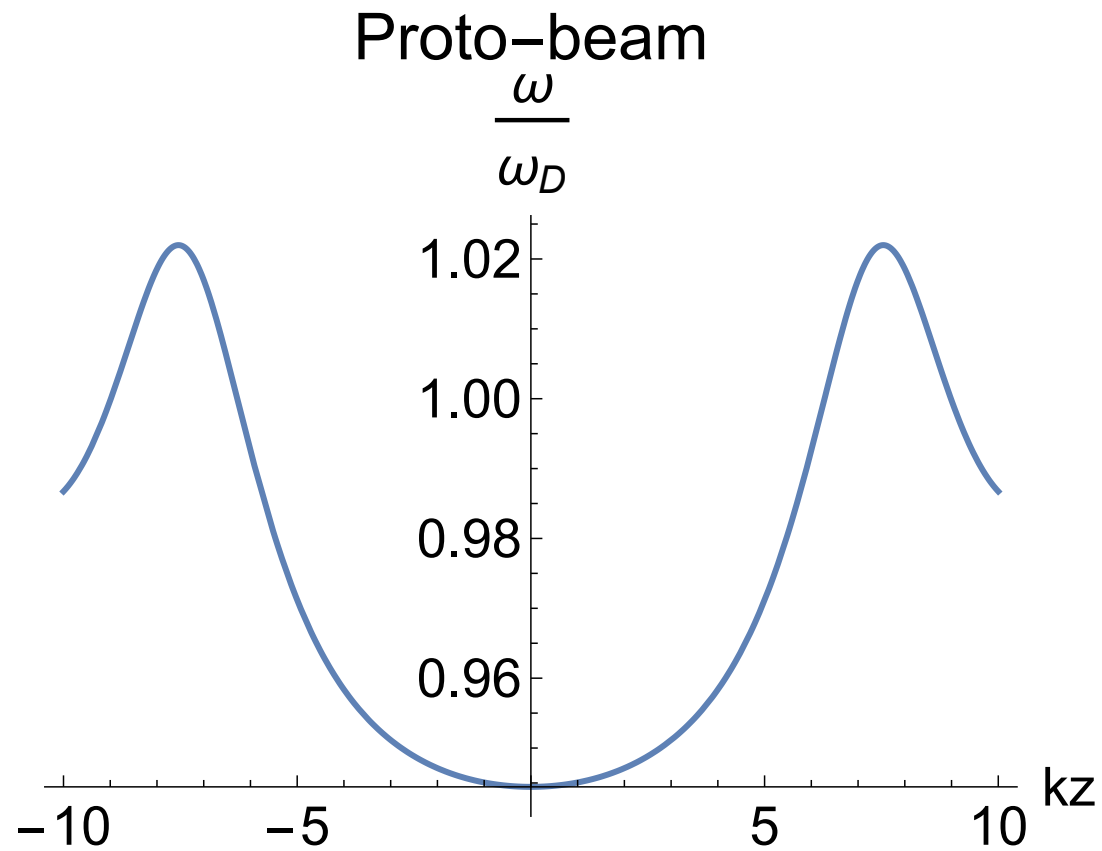

Figure 6.4: Ratio of local frequency to plane wave Doppler shifted frequency for the proto-beam. The deviation is more significant than that of a Gaussian due to the tighter focus; the tighter the beam is focused, the greater the wavelength is elongated near the focal region.

For the proto-beam for example, the $z$ derivative of the phase, and hence local wavevector is

$$
\frac{\partial S}{\partial z}=\frac{z(\sin z-z)}{z^{2}-2 z \sin z-2 \cos z+2}
$$

The local Doppler shifted frequency can be found via $\omega(z)=\gamma\left(1+\beta \frac{\partial S}{\partial z}\right)$ as usual. Figure 6.4 gives a plot of the ratio of local frequency to the Doppler frequency for the proto-beam. On the focus $z=0$, the local frequency reaches its minimum, with the ratio to the Doppler frequency given by

$$
\frac{\omega}{\omega_{D}}=\gamma\left(1+\frac{2 \beta}{3}\right) \sqrt{\frac{1-\beta}{1+\beta}} .
$$

This decreases with increasing $\beta$, reaching a minimum of $5 / 6$ at $\beta=1$. 


\subsection{Discussion}

Usually, the Doppler effect is analysed in the plane wave regime, with monochromatic beams transforming to monochromatic beams, with a shifted frequency. However, monochromacity is not a Lorentz invariant property. Frequency and wavevectors can be considered to act as a four-vector operator $k^{\mu}=-i \partial^{\mu}$, with monochromatic beams an eigenfunction of $k^{0}$. However, as this operator transforms as a four-vector, in the new frame the frequency operator will be written as

$$
k^{0^{\prime}}=\gamma\left(k^{0}-\boldsymbol{\beta} \cdot \boldsymbol{k}\right)
$$

The beam will only be an eigenfunction of this new operator if it was an eigenfunction of $\boldsymbol{k} \cdot \hat{\beta}$, i.e. is also a plane wave in the boosted direction.

A property of monochromatic beams is that their 'shape' is time independent, i.e. the modulus will be time independent. Picture for example a stationary laser experiment; the spot of the laser for all intents and purposes does not move or change shape. However, if the observer is now moving relative to the source, they must see the spot moving relative to them, mathematically, the modulus must now have some time dependence, and the beam cannot be monochromatic.

Our analysis of the Lorentz transformation of beams is not entirely novel.In particular, for the transformation of the paraxial wave equation, we mirror the approaches of Wünsche [53], Longhi [54, 56], Besieris and Shaarawi [55], who all give a similar approach to finding a polychromatic paraxial approximation. In particular, Besieris and Shaarawi converge on (6.4.10) as the relativistic paraxial equation for an envelope. It is our hope, that we have provided a simpler formulation, that is more explicitly Lorentz invariant. In particular, Besieris and Shaarawi justify neglecting the second time derivative of the envelope by assuming the wave-packet is narrowband. We do not think these are entirely equivalent assumptions, and it is our view that the simplest, and most physically relevant assumption is that the envelope varies over time scales much large than the plane wave frequency. 
Belanger [50], Zamboni-Rached and Recami [57] have done similar analysis, and concluded that under a Lorentz transformation, a monochromatic beam will become polychromatic, and the converse, that a 'pulse' can be Lorentz transformed into a monochromatic beam. We find their physical interpretations troubling however, and have also noted that Bélanger has made numerous mathematical errors in his work.. Both Belanger and Zamboni-Rached view the transformed beams as a pulse, and vice-versa. We do not believe it fruitful to view the beam as a pulse in this way: while pulses are by nature polychromatic, we believe the key property of pulses is that they are completely spatially localised. Specifically, it is our view that a pulse solution should be square integrable over all space, so that they carry finite energy, momentum etc. For Lorentz transformed beams, this is not possible: in their source frame they extend to infinity in the propagation direction, and this will still hold in the boosted frame.

It is our view that it is more fruitful to view the polychromaticity as a property of the moving envelope. As long as the phase of the envelope varies over time scales much larger than the period (which is true in the paraxial regime), one can ignore the envelopes contribution to the local frequency, and use the plane wave Doppler shift.

Finally, in this thesis we have only investigated the longitudinal Doppler shift. The beams we have considered have high symmetry about the beam axis, and thus the Lorentz transformation is simple. As an interesting example of transverse Lorentz transformation, Bliokh et al [58] investigated the transformation of a second order Bessel beam. They found that the centroid of the beam was shifted transverse to the axis and boost direction, and that the 'screw dislocation' vortex was transformed into and 'edge' dislocation. We can see there are interesting physical effects under transverse Lorentz transformation, and we would be particularly interested in the effect on the ring zeros. 


\section{Chapter 7}

\section{Covariant Hertz Potentials}

The following chapter is a modified version of a preprint I have authored as part of my Masters research, 'Lorentz transformation of electromagnetic pulses derived from Hertz potentials', accessible at arxiv.org/abs/1706.09513.

\section{Abstract}

Electric and magnetic Hertz potentials are a formalism for obtaining solutions of Maxwell's equations from solutions of the inhomogeneous wave equation, with polarisation and magnetisation as the sources. We provide an overview of their covariant transformation properties, and examine an application of Hertz potentials to the Lorentz transformation of localised pulses by Lekner, who obtained a result seeming to contradict Von Laue's theorem. We show that Lekner's result of total energy-momentum sometimes not transforming as a four-vector was due to an erroneous transformation of Hertz potentials, that did not take into account their bivector nature. 


\subsection{Introduction}

In the covariant formulation of electromagnetism, the energy and momentum density do not constitute a four vector, and instead are components of the electromagnetic stress-energy tensor, $T^{\alpha \beta}$ (Jackson [18], Chap. 12). Similarly, the angular momentum is not an anti-symmetric rank 2 tensor, but is instead given by the rank 3 tensor

$$
M^{\alpha \beta \sigma}=x^{\alpha} T^{\beta \sigma}-x^{\beta} T^{\alpha \sigma} .
$$

However, in the absence of sources (i.e. current and charge densities), the stressenergy and angular momentum tensors are divergence-less,

$$
\partial_{\alpha} T^{\alpha \beta}=0, \quad \partial_{\sigma} M^{\alpha \beta \sigma}=0 .
$$

We can define the quantities

$$
\begin{aligned}
P^{\alpha} & =\int_{\mathscr{S}} T^{\alpha \beta} \hat{e}_{\beta}^{0} \mathrm{~d}^{3} r, \\
L^{\alpha \beta} & =\int_{\mathscr{S}} M^{\alpha \beta \sigma} \hat{e}_{\sigma}^{0} \mathrm{~d}^{3} r,
\end{aligned}
$$

where $\mathscr{S}$ is a space-like hyper-surface, and $\hat{e}^{0}$ is the unit normal to $\mathscr{S}$. For the case where $\mathscr{S}$ is a hyper-surface of constant time, this will correspond to the total energy-momentum, and total angular momentum of the fields. Because of the zero divergence, it follows from Gauss' law that these integrals are independent of the choice of hyper-surface [Møller, 59, pp 166-168]. This is known as Von Laue's theorem [60], and implies that the totals are time independent.

Next we shall provide a brief overview of Hertz potentials, as given by Essex [61]. Hertz potentials allow one to easily obtain solutions of Maxwell's equations from the scalar wave equation. The formulation is as follows: let $\boldsymbol{\Pi}_{e}, \boldsymbol{\Pi}_{m}$ be two vector fields. The scalar and vector electromagnetic potentials can then be obtained as follows:

$$
\begin{gathered}
\phi=-\nabla \cdot \boldsymbol{\Pi}_{e}, \\
\boldsymbol{A}=\frac{1}{c} \frac{\partial \boldsymbol{\Pi}_{e}}{\partial t}+\nabla \times \boldsymbol{\Pi}_{m} .
\end{gathered}
$$


In order to satisfy Maxwell's equations, Hertz vectors satisfy the inhomogeneous wave equations

$$
\begin{aligned}
& \square \boldsymbol{\Pi}_{e}=4 \pi \boldsymbol{P}, \\
& \square \boldsymbol{\Pi}_{m}=4 \pi \boldsymbol{M} .
\end{aligned}
$$

where $\boldsymbol{P}, \boldsymbol{M}$ are the polarisation and magnetisation vectors respectively. In free space, it is thus sufficient that every component of the Hertz vectors satisfies the homogeneous wave equation.

It is known however, that the covariant polarisation and magnetisation are to be expressed as an antisymmetric tensor (Vanderlinde [62], p. 318),

$$
\mathscr{M}^{\alpha \beta}=\left(\begin{array}{cccc}
0 & P_{x} & P_{y} & P_{z} \\
-P_{x} & 0 & M_{z} & -M_{y} \\
-P_{y} & -M_{z} & 0 & M_{x} \\
-P_{z} & M_{y} & -M_{x} & 0
\end{array}\right) .
$$

This is due to the interchange of electric and magnetic phenomena under Lorentz transformations: as the electric and magnetic fields mix under Lorentz transformations, so must the polarisation and magnetisation. This implies that the covariant formulation of Hertz potentials is given by

$$
\Pi^{\alpha \beta}=\left(\begin{array}{cccc}
0 & \Pi_{e x} & \Pi_{e y} & \Pi_{e z} \\
-\Pi_{e x} & 0 & \Pi_{m z} & -\Pi_{m y} \\
-\Pi_{e y} & -\Pi_{m z} & 0 & \Pi_{m x} \\
-\Pi_{e z} & \Pi_{m y} & -\Pi_{m x} & 0
\end{array}\right) .
$$

This approach is given in Post [63], p. 68. It is also noted by Cohen and Kegeles [64], who additionally give the curved space-time generalisation. The Hertz tensor satisfies the inhomogeneous wave equation

$$
\square \Pi^{\alpha \beta}=4 \pi \mathscr{M}^{\alpha \beta} .
$$

The four-potential and fields are then obtained via

$$
\begin{gathered}
A^{\alpha}=\partial_{\beta} \Pi^{\alpha \beta}, \\
F^{\alpha \beta}=\partial^{\alpha} \partial_{\sigma} \Pi^{\beta \sigma}-\partial^{\beta} \partial_{\sigma} \Pi^{\alpha \sigma} .
\end{gathered}
$$


We note that when expanded into three-vector notation, that this is equivalent to 7.1 .5 . Since $\Pi$ is antisymmetric, and mixed partial derivatives commute, we have

$$
\partial_{\alpha} \partial_{\beta} \Pi^{\alpha \beta}=0=\partial_{\alpha} A^{\alpha},
$$

hence the Lorenz gauge condition is automatically satisfied. This simplifies the expression for the field tensor to

$$
F^{\alpha \beta}=\partial^{\alpha} \partial_{\sigma} \Pi^{\chi \beta}
$$

Lekner [65] employs the magnetic Hertz vector to construct electromagnetic pulses from a single pulse-like wavefunction. We will set $c=1$, and define:

$$
\begin{array}{ll}
\rho=\sqrt{x^{2}+y^{2}}, & z_{-}=z-t, \\
r=\sqrt{\rho^{2}+z^{2}}, & z_{+}=z+t .
\end{array}
$$

We will examine two of Lekner's pulses, one of which Lekner finds to be in contradiction of Von Laue's theorem. Both have a wavefunction given by a $\mathrm{Zi}$ olkowski [51] type solution,

$$
\psi=\frac{a b}{\rho^{2}+\left(a-i z_{+}\right)\left(b+i z_{-}\right)} .
$$

The two pulses are the 'CP' and TE pulses, with respective magnetic Hertz potentials given by

$$
\begin{aligned}
& \boldsymbol{\Pi}_{C P}=(i, 1,0) \psi, \\
& \boldsymbol{\Pi}_{T E}=(0,0,1) \psi .
\end{aligned}
$$

Lekner shows that these pulses can have total momenta less than $c^{-1}$ times their total energies. This implies that a frame can be found where the total momentum is zero. The energy-momentum densities for a general free space solution of Maxwell's equations satisfy (Theory of Reflection [8], p. 493)

$$
u^{2}-p^{2} c^{2} \geq 0
$$


The energy density is positive definite, and thus the total energy must be strictly greater than zero. However, if the momentum density is completely symmetric, then when integrated over all space it will cancel, leaving a zero net momentum. This can be true even if (7.1.17) is an equality everywhere. Hence, a sufficient condition for a frame with zero total momentum is a frame where the momentum is symmetrically distributed.

Maxwell's equations are still satisfied under the duality transformation [Jackson, 18, p.274]

$$
\begin{aligned}
& \boldsymbol{B} \rightarrow \boldsymbol{E}^{\prime}, \\
& \boldsymbol{E} \rightarrow-\boldsymbol{B}^{\prime},
\end{aligned}
$$

so from given fields $\boldsymbol{E}_{0}, \boldsymbol{B}_{0}$, we are free to construct a new pulse with fields given by

$$
\begin{aligned}
& \boldsymbol{B}=\boldsymbol{B}_{0}-i \boldsymbol{E}_{0}, \\
& \boldsymbol{E}=\boldsymbol{E}_{0}+i \boldsymbol{B}_{0},
\end{aligned}
$$

and this will still be a valid solution of Maxwell's equations. Such a pulse is self-dual. Since this results in $\boldsymbol{E}=i \boldsymbol{B}$, if we take the real parts of the fields and calculate the energy and momentum densities, they have the simple expression

$$
\begin{gathered}
u=\frac{1}{8 \pi}\left(\boldsymbol{E}_{r}^{2}+\boldsymbol{B}_{r}^{2}\right)=\frac{1}{8 \pi} \boldsymbol{B} \cdot \boldsymbol{B}^{*}, \\
\boldsymbol{s}=\frac{1}{4 \pi} \boldsymbol{E}_{r} \times \boldsymbol{B}_{r}=\frac{1}{4 \pi} \boldsymbol{B}_{r} \times \boldsymbol{B}_{i}=\frac{i}{8 \pi} \boldsymbol{B} \times \boldsymbol{B}^{*} d,
\end{gathered}
$$

where the star denotes complex conjugation. 


\section{2 'CP' pulse}

For the self-dual 'CP' pulse, the complex fields are given by

$$
\begin{gathered}
B_{x}=\frac{4 i a b\left(\left(b+i z_{-}\right)^{2}+\rho^{2} e^{-2 i \phi}\right)}{\alpha^{3}}, \\
B_{y}=\frac{4 a b\left(\left(b+i z_{-}\right)^{2}-\rho^{2} e^{2 i \phi}\right)}{\alpha^{3}}, \\
B_{z}=\frac{8 a b\left(b+i z_{-}\right) \rho e^{-i \phi}}{\alpha^{3}}, \\
\boldsymbol{E}=i \boldsymbol{B}, \\
\alpha \equiv a b+i a z_{-}-i b z_{+}+r^{2}-t^{2} .
\end{gathered}
$$

For the energy and momentum densities, see Appendix 7.5.

To find the totals, we integrate the densities over the hyper-surface $t=0$. This can be achieved using spherical coordinates. These integrals have closedform solutions, and give total energy and momenta of:

$$
\begin{gathered}
U=\frac{(a+3 b) \pi}{8 a^{2}}, \\
S_{x}=S_{y}=0, \\
S_{z}=\frac{(a-3 b) \pi}{8 a^{2}}, \\
U^{2}-S^{2}=\frac{3 b \pi^{2}}{16 a^{3}} .
\end{gathered}
$$

in agreement with Lekner, 2013 [66]. Note our convention is to use lower case for the densities, and upper case for the totals.

\subsubsection{Zero Momentum Frame}

As we already have explicit expressions for the fields, we shall Lorentz transform via the usual formula (Jackson [18], p. 558)

$$
\begin{aligned}
& \boldsymbol{E}^{\prime}=\gamma(\boldsymbol{E}+\boldsymbol{\beta} \times \boldsymbol{B})-\frac{\gamma^{2}}{\gamma+1} \boldsymbol{\beta}(\boldsymbol{\beta} \cdot \boldsymbol{E}), \\
& \boldsymbol{B}^{\prime}=\gamma(\boldsymbol{B}-\boldsymbol{\beta} \times \boldsymbol{E})-\frac{\gamma^{2}}{\gamma+1} \boldsymbol{\beta}(\boldsymbol{\beta} \cdot \boldsymbol{B}) .
\end{aligned}
$$


As we are dealing with fields in space-time we must also substitute the old coordinates as functions of the new coordinates, as usual. We will be boosting along $z$, and as our pulse satisfies $\boldsymbol{E}=i \boldsymbol{B}$ in all frames, it will suffice to find $\boldsymbol{B}$ in the new frame. This is given by

$$
\begin{gathered}
\bar{B}_{x}=\gamma\left(B_{x}+\beta E_{y}\right)=\gamma\left(B_{x}+i \beta B_{y}\right), \\
\bar{B}_{y}=\gamma\left(B_{y}-\beta E_{x}\right)=\gamma\left(B_{y}-i \beta B_{x}\right), \\
\bar{B}_{z}=B_{z} .
\end{gathered}
$$

As the total $x$ and $y$ momenta are zero, we can attempt to find a boost along $z$ that will bring the total momentum is zero. Letting an over-bar denote quantities in the zero momentum frame. The boost velocity is found by solving

$$
\bar{S}_{z}=\gamma\left(S_{z}-\beta U\right)=0,
$$

which gives us

$$
\beta=\frac{a-3 b}{a+3 b}
$$

Note that as we are constrained via $|\beta|<1$, we must have $|a-3 b|<|a+3 b|$. Also note that if $a=3 b$ the pulse already has zero momentum. The Lorentz transformed fields come out to be:

$$
\begin{gathered}
\bar{B}_{x}=\frac{12 i a^{2} b^{3}\left(a b-3\left(\bar{z}_{-}^{2}-\rho^{2} e^{-2 i \phi}\right)+2 i \sigma \bar{z}_{-}\right)}{\bar{\alpha}^{3}}, \\
\bar{B}_{y}=\frac{12 a^{2} b^{3}\left(a b-3\left(\bar{z}_{-}^{2}+\rho^{2} e^{-2 i \phi}\right)+2 i \sigma \bar{z}_{-}\right)}{\bar{\alpha}^{3}}, \\
\bar{B}_{z}=\frac{24 a^{2} b^{3} \rho e^{-i \phi}\left(\sigma+3 i \bar{z}_{-}\right)}{\bar{\alpha}^{3}}, \\
\overline{\boldsymbol{E}}=i \overline{\boldsymbol{B}} \\
\bar{\alpha} \equiv \sigma\left(a b\left(1+\frac{i}{\sigma}\left(3 \bar{z}_{-}-\bar{z}_{+}\right)\right)+\bar{r}^{2}-\bar{t}^{2}\right), \\
\sigma \equiv \sqrt{3 a b} .
\end{gathered}
$$

We can once again find the densities using equations (7.1.21) and (7.1.22), listed 
in Appendix 7.5. When integrated, we get totals of

$$
\begin{gathered}
\bar{U}=\frac{\pi}{4} \sqrt{\frac{3 b}{a^{3}}}=\gamma\left(U-\beta S_{z}\right), \\
\bar{S}=0, \\
\bar{U}^{2}-\overline{\boldsymbol{S}}^{2}=\frac{3 b \pi^{2}}{16 a^{3}}=U^{2}-S^{2} .
\end{gathered}
$$

Thus, we can see that the total energy momentum has transformed as a fourvector, and Von Laue's Theorem holds. Note that this is not in agreement with Lekner, 2013 [66], which seems to show the pulse violating Von Laue's theorem. We shall discuss this further in Section 7.4 .

\subsubsection{Angular momentum}

The total angular momentum is found via equations (7.1.1) and (7.1.4). The resulting tensor is antisymmetric, and will have 6 independent components. The space-space components are the total angular momenta,

$$
\boldsymbol{L}=\int_{S} \boldsymbol{x} \times \boldsymbol{p} \mathrm{d}^{3} r=\frac{1}{4 \pi} \int_{S} \boldsymbol{x} \times(\boldsymbol{E} \times \boldsymbol{B}) \mathrm{d}^{3} r .
$$

The time-space components are sometimes referred to as the 'boost angular momentum' [67], and are related to the motion of the centre of energy [68].

$$
\boldsymbol{N}=\int_{S} u \boldsymbol{x}-c t \boldsymbol{p} \mathrm{d}^{3} r=\frac{1}{4 \pi} \int_{S} \frac{E^{2}+B^{2}}{2} \boldsymbol{x}-t \boldsymbol{E} \times \boldsymbol{B} \mathrm{d}^{3} r .
$$

We can see that in the case of a $t=0$ hyper-surface, this reduces to

$$
\boldsymbol{N}=\int_{t=0} u \boldsymbol{x} \mathrm{d}^{3} r=\frac{1}{8 \pi} \int_{t=0}\left(E^{2}+B^{2}\right) \boldsymbol{x} \mathrm{d}^{3} r .
$$

In our case, in the original frame the boost angular momentum is zero, and the total angular momentum is given by

$$
\boldsymbol{L}=\frac{\pi b}{4 a} \hat{z} .
$$


As both the total angular momentum and electromagnetic fields are given by antisymmetric rank two tensors, the Lorentz transformation of the mass moment is analogous to that of the electric field, and the angular momentum analogous to that of the magnetic field. As such, we can simply use 7.2.5), under the substitution

$$
\begin{gathered}
E \rightarrow N, \\
B \rightarrow L .
\end{gathered}
$$

This means that since $\boldsymbol{L}$ is along $z$, and $\boldsymbol{N}$ is zero, the total angular momentum tensor of the pulse is unchanged by boosts along $z$.

\subsection{TE+iTM pulse}

Next, we consider the pulse given by (7.1.16). From this TE pulse, we shall construct the self-dual TE+iTM pulse via (7.1.20). The (complex) fields are then given by

$$
\begin{gathered}
B_{x}=\frac{4 a b \rho\left(e^{-i \phi}\left(z_{+}+i a\right)-e^{i \phi}\left(z_{+}+i b\right)\right)}{\alpha^{3}} \\
B_{y}=\frac{4 a b \rho\left(e^{-i \phi}\left(a-i z_{+}\right)+e^{i \phi}\left(b+i z_{-}\right)\right)}{\alpha^{3}} \\
B_{z}=\frac{4 a b\left(\left(a-i z_{+}\right)\left(b+i z_{-}\right)-\rho^{2}\right)}{\alpha^{3}} \\
\boldsymbol{E}=i \boldsymbol{B} \\
\alpha \equiv a b+i a z_{-}-i b z_{+}+r^{2}-t^{2} .
\end{gathered}
$$

The expressions for the momentum densities are unwieldy, and shall be omitted, but can easily be obtained via equations (7.1.21) and (7.1.22). When inte- 
grated the totals are given by

$$
\begin{gathered}
U=\frac{\pi(a+b)}{8 a b}, \\
S_{x}=S_{y}=0, \\
S_{z}=\frac{\pi(a-b)}{8 a b}, \\
U^{2}-S^{2}=\frac{\pi^{2}}{16 a b},
\end{gathered}
$$

reproducing Lekner, 2003 [65]. The total angular momentum however, is zero, in both the rotational and boost components.

\subsubsection{Zero momentum frame}

In this case, we can see that if $a=b$ we are already in the zero momentum frame. Otherwise, a boost of

$$
\boldsymbol{\beta}=\frac{a-b}{a+b} \hat{z}
$$

will put the pulse in its zero momentum frame. Note that the constraint for $|\beta|<1$ gives us the constraint $|a-b|<|a+b|$ for the boost to be valid. Once again, we use (7.2.5) to transform the fields. The resulting expressions are

$$
\begin{gathered}
\bar{B}_{x}=\frac{8\left(y+\frac{1}{\xi}(\bar{z} x-i \bar{t} y)\right)}{\bar{\alpha}^{3}}, \\
\bar{B}_{y}=\frac{8\left(x-\frac{1}{\xi}(\bar{z} y+i \bar{t} x)\right)}{\bar{\alpha}^{3}}, \\
\bar{B}_{z}=\frac{4\left(\xi-2 i \bar{t}-\frac{1}{\xi}\left(\rho^{2}-\bar{z}_{+} \bar{z}_{-}\right)\right)}{\bar{\alpha}^{3}}, \\
\overline{\boldsymbol{E}}=i \overline{\boldsymbol{B}} \\
\bar{\alpha} \equiv \xi-2 i \bar{t}+\frac{\bar{r}^{2}-t^{2}}{\xi}, \\
\xi \equiv \sqrt{a b} .
\end{gathered}
$$


The expressions for the densities are once again unwieldy, however the values at $t=0$ are given by

$$
\begin{gathered}
u=\frac{2 a^{2} b^{2}}{\pi(a b+\bar{r})^{4}}, \\
\boldsymbol{s}=0 .
\end{gathered}
$$

When integrated this gives

$$
\begin{gathered}
\bar{U}=\frac{\pi}{4 \sqrt{a b}}, \\
\overline{\boldsymbol{S}}=0 \\
\bar{U}^{2}-\overline{\boldsymbol{S}}^{2}=U^{2}-\boldsymbol{S}^{2}=\frac{\pi^{2}}{16 a b} .
\end{gathered}
$$

This is is agreement with Lekner 2013 [66]. As the momentum density is zero, the rotational angular momentum will also be zero, and as the energy density is spherically symmetric, when we integrate the boost angular momentum, it will be zero,

$$
\overline{\boldsymbol{N}}=\int_{\bar{t}=0} u \overline{\boldsymbol{x}} \mathrm{d}^{3} \bar{r}=0 .
$$

Due to Von Laue's theorem, the total momenta and angular momenta are conserved quantities, thus the total angular momenta are zero for all time.

\subsection{Discussion}

We have seen that Lekner's result for the Lorentz transformation of the ' $\mathrm{CP}$ ' pulse was in error, this was due to neglecting the bivector nature of the Hertz potentials. To properly Lorentz transform Hertz potentials, one should use (7.2.5), under the substitution

$$
\boldsymbol{E} \rightarrow \boldsymbol{\Pi}_{e}, \quad \boldsymbol{B} \rightarrow \boldsymbol{\Pi}_{m}
$$

For both pulses, Lekner transformed the Hertz potentials via

$$
\begin{aligned}
\boldsymbol{\Pi}_{m}(x) & \rightarrow \overline{\boldsymbol{\Pi}}_{m}(\bar{x})=\boldsymbol{\Pi}_{m}(x(\bar{x})), \\
\boldsymbol{\Pi}_{e}(x) & \rightarrow \overline{\boldsymbol{\Pi}}_{e}(\bar{x})=\boldsymbol{\Pi}_{e}(x(\bar{x})) .
\end{aligned}
$$


This is correct for the TE+iTM pulse, as in that case $\boldsymbol{\Pi}_{m}$ was parallel to the boost direction, with $\Pi_{e}=0$. This is analogous to how a boost parallel to a magnetic field, with zero electric field, will not change the fields. However, for the 'CP' pulse, this is incorrect. $\boldsymbol{\Pi}_{m}$ is perpendicular to the boost; thus not only will $\boldsymbol{\Pi}_{m}$ be strengthened by a factor of $\gamma$, but $\Pi_{e}$ will no longer be zero.

\subsection{Appendix: 'CP' momentum densities}

For the 'CP' pulse in the original frame, the four momentum densities are given by

$$
\begin{gathered}
u=\frac{4 a^{2} b^{2}\left(b^{2}+\rho^{2}+z_{-}^{2}\right)^{2}}{\pi|\alpha|^{6}}, \\
s_{x}=-\frac{8 a^{2} b^{2}\left(b^{2}+\rho^{2}+z_{-}^{2}\right)\left(b y+z_{-} x\right)}{\pi|\alpha|^{6}}, \\
s_{y}=\frac{8 a^{2} b^{2}\left(b^{2}+\rho^{2}+z_{-}^{2}\right)\left(b x-z_{-} y\right)}{\pi|\alpha|^{6}}, \\
s_{z}=-\frac{4 a^{2} b^{2}\left(b^{2}-\rho^{2}+z_{-}^{2}\right)\left(b^{2}+\rho^{2}+z_{-}^{2}\right)}{\pi|\alpha|^{6}} . \\
\alpha \equiv a b+i a z_{-}-i b z_{+}+r^{2}-t^{2} .
\end{gathered}
$$

In the zero momentum frame, the densities are given by:

$$
\begin{gathered}
\bar{u}=\frac{36 a^{4} b^{6}\left(a b+3\left(\bar{z}_{-}^{2}+\rho^{2}\right)\right)^{2}}{\pi|\bar{\alpha}|^{6}}, \\
\bar{s}_{x}=-\frac{72 a^{4} b^{6}\left(a b+3\left(\bar{z}_{-}^{2}+\rho^{2}\right)\right)\left(3 \bar{z}_{-} x+\sigma y\right)}{\pi|\bar{\alpha}|^{6}}, \\
\bar{s}_{y}=\frac{72 a^{4} b^{6}\left(a b+3\left(\bar{z}_{-}^{2}+\rho^{2}\right)\right)\left(\sigma x-3 \bar{z}_{-} y\right)}{\pi|\bar{\alpha}|^{6}}, \\
\bar{s}_{z}=-\frac{36 a^{4} b^{6}\left(a b+3\left(\bar{z}_{-}^{2}+\rho^{2}\right)\right)\left(a b+3\left(\bar{z}_{-}^{2}-\rho^{2}\right)\right)}{\pi|\bar{\alpha}|^{6}} . \\
\bar{\alpha}=\sigma\left(a b\left(1+\frac{i}{\sigma}\left(3 \bar{z}_{-}-\bar{z}_{+}\right)\right)+\bar{r}^{2}-\bar{t}^{2}\right) . \\
\sigma=\sqrt{3 a b} .
\end{gathered}
$$

The rotational and boost angular momentum densities can easily be found from the above expressions and equations (7.2.14) and (7.2.15). 


\section{Chapter 8}

\section{Single photon states}

\subsection{Introduction}

Lekner has shown that for a spatially localised classical electromagnetic pulse, the four-momentum is always time-like, $U^{2}-P^{2} c^{2}>0[8]$. This implies that a reference frame exists where a classical pulse has zero momentum. In Chapter 7 we extensively discussed the transformation properties of such pulses, and derived the energy and momentum densities for various pulse constructions. The fact that the classical pulse has a 'rest frame' must be reconciled with the fact that quantum mechanically, photons are massless. Single photon states can be found corresponding to any classical state [69]. However, the electromagnetic field of a single photon is ill-defined: the expectation value of the fields are zero. The quantum states best approximating a classical state do not have definite photon number; they are coherent states, defined as eigenstates of the photon annihilation operator. We cannot view the single photon state as having zero momentum; single photon measurements will be probabilistic, following the Born rule. As such, there will be no possible measurement that returns zero momentum. Rather, it is the expectation value that is zero, which reflects the fact that the distribution of momentum is symmetrical. 


\subsection{Construction of quantum state}

We shall employ the method of Adlard et al $[69 \mid$ of constructing single photon states that correspond to a given classical state. Working in Coulomb gauge, the four-potential of a classical solution may be written as Fourier space integral over plane wave states with a suitable polarisation basis. For plane waves along $z$, a linear polarisation basis can be taken to be $\hat{x}, \hat{y}$. We can obtain plane waves in other directions by rotating the wave-vector, and therefore can obtain the linear polarisation basis in this direction by applying the inverse of this rotation. Explicitly, this gives us

$$
\begin{gathered}
\hat{e}_{1}=(0, \cos \theta \cos \phi, \cos \theta, \sin \phi,-\sin \theta), \\
\hat{e}_{2}=(0,-\sin \phi, \cos \phi, 0), \\
\hat{k}=(0, \sin \theta \cos \phi, \sin \theta \sin \phi, \cos \theta) .
\end{gathered}
$$

One can easily verify that when $\theta, \phi=0$ these reduces to $\hat{x}, \hat{y}, \hat{z}$ respectively. Thus, the general expression for a Coulomb gauge vector potential is

$$
\hat{A}^{\mu}=\sum_{n=1,2} \int \widetilde{\mathrm{d} k} \hat{e}_{n}^{\mu}(\theta, \phi) e^{i \boldsymbol{k}(\theta, \phi) \cdot \boldsymbol{r}}\left(f_{n}(\theta, \phi) e^{-i \omega t}+g_{n}(\theta, \phi) e^{i \omega t}\right) .
$$

where the integral is over all Fourier space, $\boldsymbol{k} \in \mathbb{R}^{3}$, and $\widetilde{\mathrm{d} k}$ is the Lorentz invariant measure [Srednicki, 4, equation (3.18)]

$$
\widetilde{\mathrm{d} k}=\frac{\mathrm{d}^{3} k}{(2 \pi)^{3} 2|k|} .
$$

Causal solutions are obtained by setting $g_{n}=0$. A single photon quantum state can be obtained from this, giving the expression

$$
|\psi\rangle=\sum_{n=1,2} \int \widetilde{\mathrm{d} k} f_{n}(k) a_{n}^{\dagger}(k)|0\rangle
$$

where $a_{n}^{\dagger}(k)$ is a creation operator that creates a plane wave state with polarisation $n$ and momentum $\boldsymbol{k}(\theta, \phi)$. Note our choice of measure gives the commutator

$$
\left[a_{m}(k), a_{n}^{\dagger}(k)^{\prime}\right]=(2 \pi)^{3} 2 k \delta^{3}\left(\boldsymbol{k}-\boldsymbol{k}^{\prime}\right) \delta_{m n} .
$$


The state can be normalised if each $f_{n}$ is square integrable over Fourier space. Since the normalisation is that $\langle\psi \mid \psi\rangle=1$, this is equivalent to

$$
\int \widetilde{\mathrm{d} k}\left|f_{n}(k)\right|^{2}=1 \text {. }
$$

Such a state is a normalised single photon state, as it is an eigenvalue of the number operator

$$
\hat{N}=\sum_{n=1,2} \int \widetilde{\mathrm{d} k} a_{n}^{\dagger}(k) a_{n}(k),
$$

with eigenvalue 1 . We note that a suitable choice of $f_{n}$, such as Gaussian distributions, can yield a basis for single photon states, with the advantage of being normalisable, as opposed to the plane wave basis which is not.

Single photon states are incoherent, and have zero expectation value for the electric and magnetic fields at a given space-time point. However they do have a non-zero expectation value for their energy-momentum. For a general photon state the energy is given by the Hamiltonian

$$
\hat{H}=\sum_{n=1,2} \int \widetilde{\mathrm{d} k} \hbar \omega a_{n}^{\dagger}(k) a_{n}(k) .
$$

An eigenvalue of $\hat{H}$ is necessarily a single photon state, but the converse is not true. A single photon state is an eigenvalue of $\hat{H}$ if and only if it is monochromatic. Pulses are polychromatic, thus our single photon pulse state does not have a definite value of energy. Rather, via the Born rule, the probability of measuring the energy to be in the interval $\frac{1}{\hbar c}[\varepsilon, \epsilon+\Delta \varepsilon]$ is given by

$$
\sum_{n=1,2} \int_{|k|=\varepsilon}^{\varepsilon+\Delta \varepsilon}\left|f_{n}(k)\right|^{2} .
$$

The expectation value of energy is given by

$$
\langle E\rangle=\sum_{n=1,2} \int \widetilde{\mathrm{d} k} \hbar \omega\left|f_{n}(k)\right|^{2} .
$$

Similarly, the expectation value of momentum is given by

$$
\langle\boldsymbol{p}\rangle=\sum_{n=1,2} \int \widetilde{\mathrm{d} k} \hbar \boldsymbol{k} .
$$


Any individual measurement of momentum can only return an on-shell value. However, if the distribution of momentum is sufficiently symmetrical, the expectation value can be zero.

We now construct a single photon state from a zero-momentum classical state. We shall begin with the wavefunction of an imaginary translated spherical pulse, normalised to unity at the origin,

$$
\psi(\boldsymbol{r}, t)=\frac{\sigma^{2}-\gamma^{2}}{\rho^{2}+(z+i \gamma)^{2}-(c t+i \sigma)^{2}} .
$$

We wish to find the Fourier transform of this. We can do this for the untranslated pulse, and then use the fact that translation multiplies the Fourier transform by an exponential factor. Thus, consider a spherical pulse

$$
\Psi(\boldsymbol{r}, t)=\frac{\Psi_{0}}{r^{2}-c^{2} t^{2}} .
$$

The frequency transform gives

$$
\int_{-\infty}^{\infty} e^{i k c t} \Psi(\boldsymbol{r}, t) c \mathrm{~d} t=\bar{\Psi}(\boldsymbol{r}, k)=\Psi_{0} k \frac{\sin k r}{2 k r} \Theta(k)=\Psi_{0} \frac{k}{2} j_{0}(k r) \Theta(k) .
$$

A plane wave expansion identity is $[6$, equation (10.6.7)]

$$
e^{i \zeta \cos \alpha}=\sum_{n-0}^{\infty}(2 n+1) i^{n} j_{n}(\zeta) P_{n}(\cos \alpha),
$$

where $P_{n}$ is a Legendre polynomial. Since we have

$$
\frac{1}{2} \int_{0}^{\pi} \sin \alpha P_{n}(\cos \alpha) \mathrm{d} \alpha= \begin{cases}1, & n=0 m] \\ 0, & \text { otherwise }\end{cases}
$$

we get a useful representation of $j_{0}$ :

$$
j_{0}(k r)=\frac{1}{4 \pi} \int_{0}^{\pi} \sin \theta \mathrm{d} \theta \int_{-\pi}^{\pi} \mathrm{d} \phi e^{i \boldsymbol{k}(\theta, \phi) \cdot \boldsymbol{r}} .
$$

Thus, the Fourier transform will give us

$$
\int \mathrm{d}^{3} r j_{0}(k r) e^{-i \boldsymbol{k}^{\prime} \cdot \boldsymbol{r}}=\frac{1}{4 \pi} \int_{0}^{\pi} \int_{-\pi}^{\pi} \sin \theta \mathrm{d} \theta \mathrm{d} \phi \frac{(2 \pi)^{3}}{k^{2} \sin \theta^{\prime}} \delta\left(k-k^{\prime}\right) \delta\left(\theta-\theta^{\prime}\right) \delta\left(\phi-\phi^{\prime}\right) .
$$


Thus, in spherical coordinates, the Fourier transform of the spherical Bessel $j_{0}(k r)$ is given by

$$
\frac{2 \pi^{2}}{k^{2}} \delta\left(k-k^{\prime}\right)
$$

The final Fourier transform comes out to

$$
\tilde{\Psi}(\boldsymbol{k}, \omega)=\Psi_{0} \frac{\pi^{2}}{k} \delta\left(k-\frac{\omega}{c}\right) \Theta(\omega) .
$$

The imaginary translation multiplies by an exponential factor. Thus, we have the Fourier transform of $\psi$ given by

$$
\tilde{\psi}(\boldsymbol{k}, \omega)=\left(\sigma^{2}-\gamma^{2}\right) e^{-k(\sigma+\gamma \cos \theta)} \frac{\pi^{2}}{k} \delta\left(k-\frac{\omega}{c}\right) \Theta(\omega),
$$

where $\cos \theta=\hat{k} \cdot \hat{z}$. As has been discussed throughout the text, classical states can be constructed from a scalar wavefunction using Hertz potentials. We will be using the TE Hertz potential, that gives a vector field of

$$
\boldsymbol{A}=A_{0} \nabla \times \psi \hat{z} .
$$

In Fourier space we can represent $\nabla$ as $i \boldsymbol{k}$. We note that

$$
\boldsymbol{k} \times \hat{z}=k(\sin \theta \sin \phi,-\sin \theta \cos \phi, 0)=-k \sin \theta \hat{e}_{2}(\theta, \phi) .
$$

The TE Hertz potential therefore gives a completely linearly polarised state. For the imaginary translated spherical pulse, the vector potential is given by

$$
\begin{gathered}
\boldsymbol{A}=i A_{0} \pi^{2}\left(\gamma^{2}-\sigma^{2}\right) \int \frac{\mathrm{d}^{3} k \mathrm{~d} \omega}{(2 \pi)^{4}} \delta(k-\omega / c) \Theta(\omega) e^{-k(\sigma+\gamma \cos \theta)} \sin \theta \hat{e}_{2}(\theta, \phi) e^{i(\boldsymbol{k} \cdot \boldsymbol{r}-\omega t)} \\
=i A_{0} \pi\left(\gamma^{2}-\sigma^{2}\right) \int \widetilde{\mathrm{d} k} k \sin \theta e^{-k(\sigma+\gamma \cos \theta)} e^{i(\boldsymbol{k} \cdot \boldsymbol{r}-\omega t)} \hat{e}_{2}(\theta, \phi) .
\end{gathered}
$$

The quantum state is therefore given by

$$
|\psi\rangle=\psi_{0} \int \widetilde{\mathrm{d} k} k \sin \theta e^{-k(\sigma+\gamma \cos \theta)} a_{2}^{\dagger}(k)|0\rangle,
$$

where $\psi_{0}$ is a normalisation prefactor. The normalisation condition gives

$$
\langle\psi \mid \psi\rangle=1=\psi_{0}^{2} \int \widetilde{\mathrm{d} k} k^{2} \sin ^{2} \theta e^{-2 k(\sigma+\gamma \cos \theta)}=\frac{\psi_{0}^{2}}{16 \pi^{2}(\gamma-\sigma)^{2}(\gamma+\sigma)^{2}},
$$


the integral having required $\gamma<\sigma$ to converge. The normalisation is, up to a phase

$$
\psi_{0}=4 \pi\left(\sigma^{2}-\gamma^{2}\right)
$$

The expectation value of energy is given by

$$
\langle E\rangle=16 \pi^{2} \hbar c\left(\sigma^{2}-\gamma^{2}\right)^{2} \int \widetilde{\mathrm{d} k} k^{3} \sin ^{2} \theta e^{-k(\sigma+\gamma \cos \theta)}=\frac{2 \hbar c \sigma}{\sigma^{2}-\gamma^{2}} .
$$

The expectation value of momentum is given by

$$
\langle\boldsymbol{P}\rangle=16 \pi^{2} \hbar \hat{z}\left(\sigma^{2}-\gamma^{2}\right)^{2} \int \widetilde{\mathrm{d} k} k^{3} \sin ^{2} \theta \cos \theta e^{-k(\sigma+\gamma \cos \theta)}=\frac{2 \hbar \gamma}{\gamma^{2}-\sigma^{2}} \hat{z},
$$

where we have noted that the $x, y$ components are zero due to symmetry. We can see that when $\gamma=0$, the expectation value of momentum is zero.

Compare this with the quantities in Chapter 7, for the TE+iTM pulse. To compare the two expressions we must make the substitution $a=\sigma+\gamma$ and $b=$ $\sigma-\gamma$, in order to match the wavefunctions. Under this substitution, we have

$$
\langle E\rangle=\hbar c \frac{a+b}{a b}, \quad\langle\boldsymbol{P}\rangle=\hbar \frac{b-a}{a b} \hat{z}
$$

We can see that the functional dependence on $a, b$ matches that of the TE+iTM pulse, however the momentum is in the opposite direction. The proportionality is expected, as the method of Adlard et al is designed to reproduce the classical energy and momentum density, and thus totals. The change in sign is surprising, however. 


\section{Chapter 9}

\section{Conclusions}

In this thesis we have examined the properties of localised waves. We shall now conclude by summarising our findings, and remarking on the possible experimental implications, as well as topics for further research.

\subsection{Focusing of finite beams}

In Chapter 2 we examined how tightly finite beams can be focused. Previous theoretical bounds on beam focusing have been defined using the decay of the beam, defining spot sizes based off the radius at which the beam reaches a certain fraction of its peak. The issue with such measures however is that they are not tractable symbolically for general beams, being more useful as experimental measures. A further issue is that beams can be highly oscillatory, meaning the intensity contained within the initial spot is a small percentage of the total.

As a measure more useful for analytic results, for a wavefunction $\psi(\rho, z)$, we defined parameters $\chi$ and $\eta$,

$$
\begin{aligned}
& \chi=\frac{1}{|\psi(0,0)|^{2}} \int_{-\infty}^{\infty} \mathrm{d} z|\psi(0, z)|^{2}=\frac{2 \pi \int_{0}^{k} \mathrm{~d} q|f(k, q)|^{2}}{\left|\int_{0}^{k} \mathrm{~d} q f(k, q)\right|^{2}} \geq \frac{2 \pi}{k}, \\
& \eta=\frac{1}{|\psi(0,0)|^{2}} \int_{0}^{\infty} \mathrm{d} \rho \rho|\psi(\rho, 0)|^{2}=\frac{2 \pi \int_{0}^{k} q^{-1}|f(k, q)|^{2}}{\left|\int_{0}^{k} f(q, k)\right|^{2}} \geq \frac{4 \pi}{k^{2}},
\end{aligned}
$$


that measure the focal extent axial and radially respectively. Here we have assumed $\psi(0,0)$ is the peak of the beam. An advantage of these parameters is that they can be evaluated symbolically if the Bessel beam representation of the beam is known,

$$
\psi(\rho, z)=\int_{0}^{k} \mathrm{~d} q f(k, q) e^{i q z} J_{0}(\kappa(q) \rho),
$$

as the integrals will be equivalent into integrals of $|f(k, q)|^{2}$. The other advantage is that if the beam is centrally peaked, with this peak being the global maximum of the beam, then this measure of focal extent is entirely unambiguous.

Using the $\eta$ measure, we were able to show that the proto-beam, with a weight function $f(k, q) \propto q$, reaches the limit of tightest focus. This does not show however, that it is the unique tightly focused beam: while we are not aware of another candidate, we do not have sufficient grounds to dismiss the possibility of another weight function achieving the focusing limit.

The issue with our $\chi, \eta$ parameters is that they assume a global maximum in the modulus of the beam. For beams carrying a net angular momentum per unit length, such as 'doughnut modes', the beam can be 'hollow', with the peak of the radial profile located at some $\rho>0$. While we can still compare the radial integral to the radial peak, to define an $\eta$ value there are additional parameters we must consider. For example, should a beam with a wider peak, that is located closer to the beam axis, be considered more tightly focused than a beam with a narrower peak, further from the beam axis?

A less ambiguous measure, that is applicable to all beam geometries, is a moment based measure, such as the ratio of the first moment to the radial integral,

$$
\frac{\int_{\mathbb{R}^{2}} \mathrm{~d}^{2} r r|\psi(\rho, \phi, 0)|^{2}}{\int_{\mathbb{R}^{2}} \mathrm{~d}^{2} r|\psi(\rho, \phi, 0)|^{2}} .
$$

The issue with such measures however, is that they will not be convergent for all beams. For example, the proto-beam satisfies all criteria to be a physical beam, yet the first moment integral is divergent. In the discussion in Chapter2, we discussed the relation of moment integrals to the Laplace transform, and 
the possibility of regulating the integrals via an $e^{-s r}$ term. Evaluating at a fixed $s>0$ would give another measure of focal extent, that would be convergent for all finite beams. However, when applied to a Gaussian beam, this measure of 'spot size' approaches a fixed value of $\frac{4}{s}$ when the beam waist is taken to infinity. This limits its applicability as a focal measure. Investigation is needed into the analytic properties of such a regulator: for a parametrised family of tightly focused beams, will this regulated focal measure be asymptotic to a constant, as the focal parameter is relaxed to the limit of loosest focus?

We have defined a further focal parameter as the ratio

$$
\sigma(s)=\frac{\int_{\mathbb{R}^{2}} \mathrm{~d}^{2} r|\psi|^{2}}{\int_{\mathbb{R}^{2}} \mathrm{~d}^{2} r|\psi|^{2} e^{-s \rho}} .
$$

This parameter is guaranteed to be convergent for finite beams, and will be larger for looser focused beams. We are unable at this stage to use this to derive further rigorous results for arbitrary beams, due to the difficulty presented by Laplace transforms of products of Bessel functions. We believe it would be a useful to investigate this further, and derive rigorous focusing bounds for all beams.

\subsection{Zeros of wavefunctions}

In Chapters 3 and 4 , we presented two papers we co-authored with John Lekner, published in Optics Communications and Journal of Optics respectively. The first investigated attempts to find exact solutions of the Helmholtz equation with a Gaussian profile in their focal plane. Previous attempts used operator methods to attempt to find 'corrections' to a paraxial beam that would converge to an exact solution. We showed that such methods would in general result in evanescent beams, that have exponential growth in $z$. Such beams cannot be physical, and as such, we have shown that physical solutions cannot have a Gaussian profile in their focal plane.

More surprisingly, we were able to show that no exact solution could reproduce the Gaussian beam axially; this is surprising as the paraxial approxima- 
tion is considered to be accurate near the beam axis.

The second paper investigated the exact solution of the Helmholtz equation that is closest to the Gaussian radially,

$$
\psi_{C}(\rho, z)=\frac{b}{k} \int_{0}^{k} \mathrm{~d} \kappa \kappa e^{-\frac{b \kappa^{2}}{2 k}+i q z} J_{0}(\kappa \rho) .
$$

This beam is particularly interesting due to the behaviour of its zeros. These have creation-annihilation events at critical values of $k b$, resulting in the conversion of a pair of focal plane zeros into a pair off-plane. We investigated corresponding electromagnetic-beams and found that they have a corresponding polarisation singularities at the zeros, and these singularities have a corresponding transition.

The polarisation behaviour of general electromagnetic beams is particularly interesting in the tightly focused regime. The electric field is always locally transverse to the Poynting vector $\boldsymbol{S}=\frac{1}{4 \pi} \boldsymbol{E} \times \boldsymbol{B}$. However, the Poynting vector locally will not point along the beam axis everywhere. Three parameters are needed to describe polarisation generally; one for each axis of the polarisation ellipse, and one for the normal, which describes the direction of the plane of polarisation. When the Poynting vector deviates from the beam axis, the polarisation normal will do so also.

This raises some interesting questions; commonly linear polarisers are modelled as a planar sheet that absorbs the electric field in a particular planar direction. What happens when a significantly focused beam has its focal plane aligned with the polariser? We would expect a lower transmittance compared to the plane wave value for a focused beam simply due to reflections, however would there also be a significant deviation due to the polarisation properties?

In addition, for Gaussian beams, where the electric field is taken to be transverse to the beam axis everywhere, with uniform polarisation, one can place the polariser anywhere along the beam axis and still expect the same transmittance. On the other hand, for an exact solution of Maxwell's equations, these properties are not possible. We would expect a significant positional dependence of the transmittance of the beam based on the polariser location relative 
to the focus. This would be a particularly interesting topic to explore.

\subsection{Reflection of beams}

In Chapter 5 we examined the reflection of general particle beams off of a delta potential. We found that the position of the potential did not affect the transmittance, but that locally the zeros of the wavefunction were significantly affected. For normal incidence, we found that the zeros are deflected by the potential back toward the source, and are no longer co-linear.

We also investigated a obliquely incident Bessel beam, and found that the transmitted beam is still 'diffraction-less', remaining an eigenfunction of $\hat{k}_{z}$ with the same eigenvalue. However, the azimuthally symmetry of the beam is broken, and there is a corresponding phase singularity effect. The incident beam has ring zeros, due to the imaginary part being zero throughout the radial plane, and the real part having ring zeros. The transmitted beam has ring zeros for the real and imaginary parts, that are not coincident, rather being shifted along $\hat{x}$. At their crossing is a pair of axial vortex zeros with opposite charge.

We were able to obtain transmittances for obliquely incident general beams, in integral form. While not tractable symbolically, the expression are simple to evaluate numerically. We found that compared to the plane wave transmittance, a general beam will have a lower transmittance at normal incidence, and higher at oblique incidence. This is due to the fact that general beams are superpositions of plane waves in all possible directions along the incident hemisphere. As such, even at the perpendicular incident limit there are still some components of the beam that are forward propagating relative to the barrier.

Our investigation was limited to the Dirac delta potential, as it is the simplest exactly solvable potential. This is only an appropriate model for very thin barriers, such as oxide layers, and also assumes that the permittivity of the material is the same on either side. A more realistic potential is a step potential, which is also exactly solvable, however time did not permit this investigation. We would expect very little to change qualitatively with respect to the transmit- 
tances, as both solutions admit plane waves as their outgoing solutions.

An issue with both the delta potential and step potential is that they assume an infinite plane potential. This results in a translational independence with respect to the beam axis, as the transmittance only depends on the global properties of the incident wave. A more realistic potential would be apertured, and in this case we would expect the position of the beam focus to be far more significant. However, such a potential would be challenging to investigate analytically, and would most likely require numeric integration to calculate transmittances. This would have to be done case by case, which limits what can be said in general.

Our investigation was also limited to particle beams. For electromagnetic beams, the situation is complicated by the polarisation of the beam. As well as the direction of the wavevector, the direction of the electric field would also come into play. As a result, we would expect a significant dependence on the focal plane position; far from the focal plane the beam can be approximated as a spherical wave, and the beam can be analysed as an incident mixture of $s$ and $p$ polarised plane waves. Close to the focus however, the existence of polarisation singularities could complicate the reflection considerably.

\subsection{Lorentz transformation of localised waves}

In Chapters 6 and 7 we investigated the Lorentz transformation of beams and pulses. We found that monochromatic beams will be polychromatic in a boosted frame; in the source's rest frame, the beam is a superposition of monochromatic plane waves that travel in different directions. As a result, each mode is Doppler shifted by a different factor, and the result is a dispersion in frequency, that is related to the dispersion of the wavevector in the boost direction.

The physical significance depends on the scattering problem under consideration; for scatterers (or absorbers) significantly larger than the wavelength of the beam, the global properties, such as the frequency dispersion will be relevant. Doppler broadening in spectroscopy occurs even for plane waves due 
to the dispersion in velocities of a thermal mixture of particles. However, the Doppler broadening in our analysis has been intrinsic to the beam; as well as the whole mixture having a broadening of spectral lines due to the different Lorentz transformation of each particle, each individual particle has an associated broadening due to the beam's structure. An interesting topic for further study would be to take a model spectrum, for example a Gaussian or Lorentzian, as well as a thermodynamic particle velocity distribution, and model the total absorbance of the mixture for a particular beam. We would expect this to differ from the plane wave absorbance.

The other extreme is for particles far smaller than the wavelength of the beam. In this case it is the local frequency that will be important. Any beam can be written as an envelope times a plane wave phase. The local four-wavevector will of course transform as four-vector, and if the envelope is weakly focused, the local four-wavevector will be the very close to the four-wavevector of the plane wave phase. As a result, the deviation from the plane wave Doppler shift for the local frequency is negligible in this case. On the other hand, for a tightly focused beam, the spatial wavevector near the focal region can be considerably contracted, and the local frequency will be Doppler shifted less than the plane wave would.

\subsection{Energy and momentum of single photon pulses}

Our final chapter dealt with the energy and momentum of single photon pulses. Lekner has shown that for finite pulses (such that the total energy and momentum is finite), the total four-momentum is time-like, $U^{2}-P^{2} c^{2}>0$. This implies that for a classical pulse there is a reference frame where the total momentum is zero.

We used the Fourier decomposition of the electromagnetic field to obtain a corresponding single photon quantum electrodynamic state. Such a method results in an expectation value for four-momentum that is proportional to the classical total. In the zero-momentum frame of the pulse, the expectation value 
of momentum vanishes. However, for a quantum pulse the measurement of four-momentum is probabilistic; a pulse is not an eigenstate of the four-momentum operator. As such, any individual measurement will find a value of four-momentum that is light-like. It is in fact the symmetry of the pulse that results in zero expectation value.

The notion of a free photon can be misleading; in quantum electrodynamics photons should be taken to exist as carriers in an interaction. As such, we would expect the photon state to be a relevant description for only a finite time interval, originating at a source, and terminating at an interaction. The existence of the zero momentum frame is due to the fact that structured light can have energy transport velocities slower than $c$ (consider waveguides for example). In this zero momentum frame the observer still sees a finite lifetime for the photon, as they will still observe the creation and destruction of the photon. It is not reasonable to think of a photon as 'sitting stationary'. In addition, the energy and momentum density is not static, even in the zero momentum frame the energy density contracts to a tightest focus, then expands again 65 , just as in the source frame, the only difference being the centre of energy being constant. 


\section{Bibliography}

[1] D. J. Griffiths. Introduction to Quantum Mechanics. 2nd edition. Upper Saddle River, NJ: Pearson Prentice Hall, Apr. 10, 2004. 480 pp. ISBN: 978-0-13-111892-8.

[2] W. Greiner and J. Reinhardt. Field Quantization. Berlin, Heidelberg: Springer Berlin Heidelberg, 1996. ISBN: 978-3-540-78048-9

978-3-642-61485-9. DOI:10.1007/978-3-642-61485-9. URL: http://link.springer.com/10.1007/978-3-642-614859 (visited on $05 / 24 / 2017$ ).

[3] W. Greiner and J. Reinhardt. Quantum Electrodynamics. 4th ed. OCLC: ocn260209269. Berlin: Springer, 2009. 447 pp. ISBN: 978-3-540-87560-4 978-3-540-87561-1.

[4] M. Srednicki. Quantum Field Theory. 1 edition. Cambridge ; New York: Cambridge University Press, Feb. 5, 2007. 660 pp. ISBN: 978-0-521-86449-7.

[5] N. Young. An Introduction to Hilbert Space. Cambridge mathematical textbooks. Cambridge [England] ; New York: Cambridge University Press, 1988. 239 pp. ISBN: 978-0-521-33071-8 978-0-521-33717-5.

[6] F. W. J. Olver and National Institute of Standards and Technology (U.S.), eds. NIST Handbook of Mathematical Functions. OCLC: ocn502037224. Cambridge ; New York: Cambridge University Press : NIST, 2010. 951 pp. ISBN: 978-0-521-19225-5 978-0-521-14063-8. URL:

http://dlmf.nist.gov/. 
[7] M. I. Rabinovich and D. I. Trubetskov. Oscillations and Waves. Dordrecht: Springer Netherlands, 1989. ISBN: 978-94-010-6956-4 978-94-009-1033-1. DOI:10.1007/978-94-009-1033-1. URL: http://link.springer.com/10.1007/978-94-009-10331 (visited on $02 / 05 / 2018$ ).

[8] J. Lekner. Theory of Reflection. Vol. 87. Springer Series on Atomic, Optical, and Plasma Physics. Cham: Springer International Publishing, 2016. ISBN: 978-3-319-23626-1 978-3-319-23627-8. DOI: 10.1007/978-3-319-23627-8, uRL: http: //link.springer.com/10.1007/978-3-319-23627-8 (visited on 05/24/2017).

[9] A. E. Siegman. Lasers. University Science Books, 1986. 1322 pp. ISBN: 978-0-935702-11-8.

[10] T. Takenaka, M. Yokota, and O. Fukumitsu. "Propagation of Light Beams beyond the Paraxial Approximation". In: J. Opt. Soc. Am. A, JOSAA 2.6 (June 1, 1985), pp. 826-829. ISSN: 1520-8532. DOI:

$10.1364 /$ JOSAA.2.000826. URL: https: //www.osapublishing.org/abstract.cfm?uri=josaa-26-826(visited on 06/07/2017).

[11] S. R. Seshadri. "Fundamental Electromagnetic Gaussian Beam beyond the Paraxial Approximation”. In: J. Opt. Soc. Am. A, JOSAA 25.9 (Sept. 1, 2008), pp. 2156-2164. ISSN: 1520-8532. DOI:

$10.1364 /$ JOSAA.25.002156. URL: https: //www.osapublishing.org/abstract.cfm?uri=josaa25-9-2156 (visited on 06/07/2017).

[12] A. Wünsche. "Transition from the Paraxial Approximation to Exact Solutions of the Wave Equation and Application to Gaussian Beams”. In: JOSA A 9.5 (1992), pp. 765-774. URL: https : //www.osapublishing.org/abstract.cfm?uri=josaa-95-765 (visited on 05/24/2017). 
[13] A. April. "Nonparaxial Elegant Laguerre-Gaussian Beams". In: Optics letters 33.12 (2008), pp. 1392-1394. URL: https : //www.osapublishing.org/abstract.cfm?uri=ol-3312-1392 (visited on 06/15/2017).

[14] J. Lekner. “Tight Focusing of Light Beams: A Set of Exact Solutions”. In: Proceedings of the Royal Society A: Mathematical, Physical and Engineering Science 472.2195 (Nov. 2016), p. 20160538. ISSN: 1364-5021, 1471-2946. DOI:10.1098/rspa.2016.0538, URL: http://rspa.royalsocietypublishing.org/lookup/ doi/10.1098/rspa.2016.0538 (visited on 05/24/2017).

[15] W. H. Carter. "Anomalies in the Field of a Gaussian Beam near Focus". In: Optics Communications 7.3 (1973), pp. 211-218. URL:

http://www.sciencedirect.com/science/article/pii/ 0030401873900126 (visited on 07/23/2017).

[16] M. V. Berry. "Wave Dislocation Reactions in Non-Paraxial Gaussian Beams”. In: Journal of Modern Optics 45.9 (Sept. 1998), pp. 1845-1858. ISSN: 0950-0340, 1362-3044. DOI:10.1080/09500349808231706. URL:http://www.tandfonline.com/doi/abs/10.1080/ 09500349808231706 (visited on 07/18/2017).

[17] G. P. Karman et al. "Airy Pattern Reorganization and Subwavelength Structure in a Focus". In: J. Opt. Soc. Am. A, JOSAA 15.4 (Apr. 1, 1998), pp. 884-899. ISSN: 1520-8532. DOI: 10.1364 / JOSAA. 15.000884. URL: https: //www.osapublishing.org/abstract.cfm?uri=josaa$15-4-884$

[18] J. D. Jackson. Classical Electrodynamics. 3rd ed. Hoboken, NY: Wiley, 2007. 832 pp. ISBN: 978-81-265-1094-8.

[19] M. Born and E. Wolf. Principles of Optics: Electromagnetic Theory of Propagation, Interference and Diffraction of Light. 7th edition. 
Cambridge ; New York: Cambridge University Press, Oct. 13, 1999. 952 pp. ISBN: 978-0-521-64222-4.

[20] J. Lekner. "Polarization of Tightly Focused Laser Beams". In: J. Opt. A: Pure Appl. Opt. 5.1 (2003), p. 6. ISSN: 1464-4258. DOI: $10.1088 / 1464-4258 / 5 / 1 / 302$. URL: http://stacks.iop.org/1464-4258/5/i=1/a=302 (visited on $10 / 25 / 2017)$.

[21] J. Lekner. "Localized Electromagnetic Pulses with Azimuthal Dependence”. In: Journal of Optics A: Pure and Applied Optics 6.7 (July 1, 2004), pp. 711-716. ISSN: 1464-4258, 1741-3567. DOI:

$10.1088 / 1464-4258 / 6 / 7 / 009$, URL: http://stacks.iop.org/1464-4258/6/i=7/a=009?key= crossref.afe5b419e771e7d56a5a624730cfccd2 (visited on 05/24/2017).

[22] J. Lekner and P. Andrejić. "Nonexistence of Exact Solutions Agreeing with the Gaussian Beam on the Beam Axis or in the Focal Plane". In: Optics Communications 407 (Supplement C Jan. 15, 2018), pp. 22-26. ISSN: 0030-4018. DOI: $10.1016 / j$.optcom.2017.08.071. URL: http://www.sciencedirect.com/science/article/pii/ S0030401817307599 (visited on 10/25/2017).

[23] P. Andrejić and J. Lekner. “Topology of Phase and Polarisation Singularities in Focal Regions". In: J. Opt. 19.10 (2017), p. 105609. ISSN: 2040-8986. DOI:10.1088/2040-8986/aa895d. URL: http://stacks.iop.org/2040-8986/19/i=10/a=105609 (visited on 10/25/2017).

[24] J. Lekner. “Invariants of Atom Beams”. In: Journal of Physics B: Atomic, Molecular and Optical Physics 37.8 (2004), p. 1725. URL:

http://iopscience.iop.org/article/10.1088/09534075/37/8/013/meta (visited on 10/09/2017). 
[25] J. Lekner. "Invariants of Three Types of Generalized Bessel Beams". In: Journal of Optics A: Pure and Applied Optics 6.9 (Sept. 1, 2004), pp. 837-843. ISSN: 1464-4258, 1741-3567. DOI:

$10.1088 / 1464-4258 / 6 / 9 / 004$, URL:

http://stacks.iop.org/1464-4258/6/i=9/a=004?key= crossref.e0065aaf883b86eee102397a255f4c21 (visited on 06/29/2017).

[26] Lasers and laser-related equipment-Test methods for laser beam widths, divergence angles and beam propagation ratios - Part 2: General astigmatic beams. ISO 11146-2:2005. International Organization for Standardization, Feb. 2005.

[27] Z. Bouchal. "Nondiffracting Optical Beams: Physical Properties, Experiments and Applications". In: Czechoslovak Journal of Physics 53.7 (2003), pp. 537-578. ISSN: 00114626. DOI:

10.1023/A:1024802801048, arXiv:physics/0309109, URL: http://arxiv.org/abs/physics/0309109 (visited on 02/05/2018).

[28] D. J. Griffiths. Introduction to Electrodynamics. 4 edition. Boston: Pearson, Oct. 6, 2012. 624 pp. ISBN: 978-0-321-85656-2.

[29] R. Dorn, S. Quabis, and G. Leuchs. "Sharper Focus for a Radially Polarized Light Beam”. In: Phys. Rev. Lett. 91.23 (Dec. 2, 2003), p. 233901. DOI: $10.1103 /$ PhysRevLett.91.233901, uRL: https: //link.aps.org/doi/10.1103/PhysRevLett.91.233901 (visited on 11/03/2017).

[30] E. Kausel and M. M. Irfan Baig. "Laplace Transform of Products of Bessel Functions: A Visitation of Earlier Formulas”. In: Quarterly of Applied Mathematics 70.1 (Jan. 1, 2012), pp. 77-97. ISSN: 0033-569X, 1552-4485. DOI: $10.1090 /$ S0033-569X-2011-01239-2. URL: 
getitem?pii=S0033-569X-2011-01239-2 (visited on $01 / 18 / 2018)$.

[31] A. Zangwill. Modern Electrodynamics. Cambridge: Cambridge University Press, 2013. 976 pp. ISBN: 978-0-521-89697-9.

[32] M. Lax, W. H. Louisell, and W. B. McKnight. "From Maxwell to Paraxial Wave Optics”. In: Phys. Rev. A 11.4 (Apr. 1, 1975), pp. 1365-1370. DOI:

10.1103/PhysRevA.11.1365. URL: https://link.aps.org/doi/10.1103/PhysRevA.11.1365 (visited on 10/25/2017).

[33] G. P. Agrawal and M. Lax. "Free-Space Wave Propagation beyond the Paraxial Approximation”. In: Phys. Rev. A27.3 (Mar. 1, 1983), pp. 1693-1695. DOI:10.1103/PhysRevA.27.1693. URL: https://link.aps.org/doi/10.1103/PhysRevA.27.1693 (visited on 10/25/2017).

[34] H. Laabs. "Propagation of Hermite-Gaussian-Beams beyond the Paraxial Approximation”. In: Optics Communications 147.1 (Feb. 1, 1998), pp. 1-4. ISSN: 0030-4018. DOI:

$10.1016 / \mathrm{S} 0030-4018$ (97)00607-X. URL:

http://www.sciencedirect.com/science/article/pii/ S003040189700607X (visited on 10/25/2017).

[35] H.-C. Kim and Y. H. Lee. "Higher-Order Corrections to the Electric Field Vector of a Gaussian Beam”. In: J. Opt. Soc. Am. A, JOSAA 16.9 (Sept. 1, 1999), pp. 2232-2238. ISSN: 1520-8532. DOI:

$10.1364 /$ JOSAA.16.002232. URL: https:

//www.osapublishing.org/abstract. cfm?uri=josaa16-9-2232 (visited on 10/25/2017).

[36] A. April. "Bessel-Gauss Beams as Rigorous Solutions of the Helmholtz Equation”. In: JOSA A 28.10 (2011), pp. 2100-2107. URL: https : //www.osapublishing.org/abstract.cfm?uri=josaa28-10-2100 (visited on 06/15/2017). 
[37] F. Gori, G. Guattari, and C. Padovani. "Bessel-Gauss Beams”. In: Optics Communications 64.6 (Dec. 1987), pp. 491-495. ISSN: 00304018. DOI: $10.1016 / 0030-4018$ (87) 90276-8. URL: http://linkinghub.elsevier.com/retrieve/pii/ 0030401887902768 (visited on 06/29/2017).

[38] G. N. Watson. A Treatise on the Theory of Bessel Functions. 2nd ed., Cambridge Mathematical Library ed. Cambridge mathematical library. Cambridge [England] ; New York: Cambridge University Press, 1995. 804 pp. ISBN: 978-0-521-48391-9 978-0-521-06743-0.

[39] J. F. Nye and M. V. Berry. “Dislocations in Wave Trains”. In: Proc. R. Soc. Lond. A 336.1605 (Jan. 22, 1974), pp. 165-190. ISSN: 0080-4630, 2053-9169. DOI:10.1098/rspa.1974.0012, uRL:http://rspa . royalsocietypublishing.org/content/336/1605/165 (visited on 10/25/2017).

[40] J. F. Nye. Natural Focusing and Fine Structure of Light: Caustics and Wave Dislocations. CRC Press, Jan. 1, 1999. 354 pp. ISBN: 978-0-7503-0610-2.

[41] M. V. Berry and M. R. Dennis. "Polarization Singularities in Isotropic Random Vector Waves". In: Proceedings of the Royal Society A:

Mathematical, Physical and Engineering Sciences 457.2005 (Jan. 8, 2001), pp. 141-155. ISSN: 1364-5021, 1471-2946. DOI: $10.1098 / \mathrm{rspa} .2000 .0660$. URL: http://rspa.royalsocietypublishing.org/cgi/doi/ $10.1098 /$ rspa.2000.0660 (visited on 10/25/2017).

[42] M. R. Dennis. "Polarization Singularities in Paraxial Vector Fields: Morphology and Statistics". In: Optics Communications 213.4 (Dec. 1, 2002), pp. 201-221. ISSN: 0030-4018. DOI:

$10.1016 / \mathrm{S} 0030-4018$ (02) 02088-6. URL: http://www.sciencedirect.com/science/article/pii/ S0030401802020886 (visited on 10/25/2017). 
[43] M. V. Berry. "Index Formulae for Singular Lines of Polarization”. In: J. Opt. A: Pure Appl. Opt. 6.7 (2004), p. 675. ISSN: 1464-4258. DOI: $10.1088 / 1464-4258 / 6 / 7 / 003$, URL: http://stacks.iop.org/1464-4258/6/i=7/a=003 (visited on $10 / 25 / 2017)$.

[44] G. P. Karman et al. "Creation and Annihilation of Phase Singularities in a Focal Field”. In: Opt. Lett., OL 22.19 (Oct. 1, 1997), pp. 1503-1505. ISSN: 1539-4794. DOI:10.1364/OL.22.001503, uRL:https: //www.osapublishing.org/abstract.cfm?uri=ol-22$19-1503$.

[45] J. F. Nye. "Unfolding of Higher-Order Wave Dislocations". In: J. Opt. Soc. Am. A, JOSAA 15.5 (May 1, 1998), pp. 1132-1138. ISSN: 1520-8532. DOI: $10.1364 /$ JOSAA.15.001132, URL: https: //www.osapublishing.org/abstract.cfm?uri=josaa15-5-1132.

[46] H. Rubinsztein-Dunlop et al. "Roadmap on Structured Light”. In: Journal of Optics 19.1 (Nov. 25, 2016). ISSN: 2040-8978. URL: http://eprints.gla.ac.uk/128769/(visited on 10/25/2017).

[47] H. Hurwitz. “The Statistical Properties of Unpolarized Light”. In: J. Opt. Soc. Am., JOSA 35.8 (Aug. 1, 1945), pp. 525-531. DOI:

$10.1364 / J O S A .35 .000525$, URL: https: //www.osapublishing.org/abstract.cfm?uri=josa-358-525 (visited on 10/25/2017).

[48] M. V. Berry. "Optical Currents". In: J. Opt. A: Pure Appl. Opt. 11.9 (2009), p. 094001. ISSN: 1464-4258. DOI:

10.1088/1464-4258/11/9/094001. URL: http://stacks.iop.org/1464-4258/11/i=9/a=094001 (visited on 10/25/2017).

[49] P. A. Belanger. "Packetlike Solutions of the Homogeneous-Wave Equation”. In: JOSA A 1.7 (1984), pp. 723-724. 
[50] P. A. Belanger. "Lorentz Transformation of Packetlike Solutions of the Homogeneous-Wave Equation”. In: J. Opt. Soc. Am. A 3.4 (1986), pp. 541-542. URL: https: / / www. osapublishing.org/ viewmedia.cfm?id=59201\&seq=0 (visited on 06/19/2017).

[51] R. W. Ziolkowski. "Localized Transmission of Electromagnetic Energy". In: Physical Review A 39.4 (1989), p. 2005. URL: https : / journals . aps.org/pra/abstract/10.1103/PhysRevA.39.2005 (visited on 06/06/2017).

[52] R. W. Ziolkowski. "New Electromagnetic Directed Energy Pulses”. In: vol. 0873. 1988, pp. 312-319. DOI: 10 .1117/12 .965110. URL: http://dx.doi.org/10.1117/12.965110.

[53] A. Wünsche. "Embedding of Focus Wave Modes into a Wider Class of Approximate Wave Equation Solutions". In: Journal of the Optical Society of America A 6.11 (Nov. 1, 1989), p. 1661. ISSN: 1084-7529, 1520-8532. DOI:10.1364/JOSAA.6.001661, uRL: https: //www.osapublishing.org/abstract.cfm?URI=josaa-611-1661 (visited on 02/12/2018).

[54] S. Longhi. “Gaussian Pulsed Beams with Arbitrary Speed”. In: Optics Express 12.5 (2004), pp. 935-940.

[55] I. M. Besieris and A. M. Shaarawi. "Paraxial Localized Waves in Free Space”. In: Optics Express 12.16 (2004), pp. 3848-3864.

[56] S. Longhi. "Gauss-Laguerre Nondispersive and Nondiffractive Pulsed Beams in Dispersive Media”. In: Conference on Lasers and Electro-Optics, 2004. (CLEO). Conference on Lasers and Electro-Optics, 2004. (CLEO). Vol. 1. May 2004, 2 pp. vol.1-.

[57] M. Zamboni-Rached and E. Recami. "Sub-Luminal Wave Bullets: Exact Localized Subluminal Solutions to the Wave Equations". In: Physical Review A77.3 (Mar. 11, 2008). ISSN: 1050-2947, 1094-1622. DOI: 10.1103/PhysRevA.77.033824, arXiv:0709.2372, URL: http://arxiv.org/abs/0709.2372. 
[58] K. Y. Bliokh and F. Nori. "Relativistic Hall Effect". In: Physical Review Letters 108.12 (Mar. 21, 2012). ISSN: 0031-9007, 1079-7114. DOI: 10.1103 /PhysRevLett.108.120403, arXiv: 1112.5618, URL: http://arxiv.org/abs/1112.5618.

[59] C. Møller. The Theory Of Relativity. Clarendon Press, 1952. 409 pp. URL: http: //archive.org/details/theoryofrelativi029229mbp.

[60] D. J. Griffiths. "Resource Letter EM-1: Electromagnetic Momentum”. In: American Journal of Physics 80.1 (2012), pp. 7-18. URL: http: //aapt.scitation.org/doi/abs/10.1119/1.3641979 (visited on 05/24/2017).

[61] E. A. Essex. "Hertz Vector Potentials of Electromagnetic Theory". In: American Journal of Physics 45.11 (Nov. 1, 1977), pp. 1099-1101. ISSN: 0002-9505. DOI:10.1119/1.10955. URL: http://aapt.scitation.org.helicon.vuw.ac.nz/doi/ abs/10.1119/1.10955.

[62] J. Vanderlinde. Classical Electromagnetic Theory. Springer Science \& Business Media, Jan. 17, 2006. 427 pp. ISBN: 978-1-4020-2700-0.

[63] E. J. Post. “General Covariance in Electromagnetism”. In: Delaware Seminar in the Foundations of Physics. Studies in the Foundations Methodology and Philosophy of Science. Springer, Berlin, Heidelberg, 1967, pp. 102-123. ISBN: 978-3-642-86104-8 978-3-642-86102-4. DOI: $10.1007 / 978-3-642-86102-4 \_7$. URL: https://link.springer.com/chapter/10.1007/978-3642-86102-4_7 (visited on 06/26/2017).

[64] J. M. Cohen and L. S. Kegeles. "Electromagnetic Fields in Curved Spaces: A Constructive Procedure”. In: Phys. Rev. D 10.4 (Aug. 15, 1974), pp. 1070-1084. DOI:10.1103/PhysRevD.10.1070, URL: https://link.aps.org/doi/10.1103/PhysRevD.10.1070, 
[65] J. Lekner. "Electromagnetic Pulses Which Have a Zero Momentum Frame”. In: Journal of Optics A: Pure and Applied Optics 5.4 (2003), p. L15. URL:

http://iopscience.iop.org/article/10.1088/14644258/5/4/101/meta (visited on 05/24/2017).

[66] J. Lekner. “Universal Properties of Electromagnetic Pulses”. In: PIERS Proceedings, Taipei (2013), pp. 458-463. URL: https : //piers.org/piersproceedings/download.php?file= cGllcnMyMDEzVGFpcGVpfDJQNF 8wNDU4 LnBkZnwxMjEwMDQxOT IxNDU= (visited on 05/24/2017).

[67] R. P. Cameron et al. "Chirality and the Angular Momentum of Light". In: Philos Trans A Math Phys Eng Sci 375.2087 (Feb. 28, 2017). ISSN: 1364-503X. DoI:10.1098/rsta.2015.0433.pmid:28069764. URL: http: //www.ncbi.nlm.nih.gov/pmc/articles/PMC5247477/ (visited on 05/24/2017).

[68] M. H. L. Pryce. "The Mass-Centre in the Restricted Theory of Relativity and Its Connexion with the Quantum Theory of Elementary Particles". In: Proceedings of the Royal Society of London A: Mathematical, Physical and Engineering Sciences. Vol. 195. The Royal Society, 1948, pp. 62-81. URL: http: / / rspa.royalsocietypublishing.org/ content/195/1040/62. short (visited on 05/25/2017).

[69] C. Adlard, E. R. Pike, and S. Sarkar. "On the Localization of One-Photon States”. In: Physical Review Letters 79.9 (Sept. 1, 1997), pp. 1585-1587. ISSN: 0031-9007, 1079-7114. DOI:

10.1103/PhysRevLett. 79.1585, arXiv: quant-ph/9707027. URL: http://arxiv.org/abs/quant-ph/9707027/visited on 05/25/2017). 